\title{
8th International Symposium on Resistance Arteries
}

New Developments in Resistance Artery Research. Molecular Biology to Bedside

(www.isra2004.org)

Angers, France

June 20-23, 2004

Guest Editor

Daniel Henrion, Angers, France

95 abstracts, PDF (838 Kb), 2004 
S. Karger

Medical and Scientific Publishers Basel $\cdot$ Freiburg $\cdot$ Paris $\bullet$ London New York $\bullet$ Bangalore $\cdot$ Bangkok Singapore $\bullet$ Tokyo $\bullet$ Sydney
Drug Dosage

The authors and the publisher have exerted every effort to ensure that drug selection and dosage set forth in this text are in accord with current recommendations and practice at the time of publication. However, in view of ongoing research, changes in government regulations, and the constant flow of information relating to drug therapy and drug reactions, the reader is urged to check the package insert for each drug for any change urged to check the package insert for each drug for any change
in indications and dosage and for added warnings and precautions. This is particularly important when the recommended agent is a new and/or infrequently employed drug.
All rights reserved.

No part of this publication may be translated into other languages, reproduced or utilized in any form or by any means, electronic or mechanical, including photocopying, recording, microcopying, or by any information storage and retrieval system, without permission in writing from the publisher or, in the case of photocopying, direct payment of a specified fee to the Copyright Clearance Center (see 'General Information').

(C) Copyright 2004 by S. Karger AG

P.O. Box, CH-4009 Basel (Switzerland)

Printed in Switzerland on acid-free paper by free electronic supplement:

www.karger.com/jvr_ISRA2004

\section{KARGER}

Fax +4161306 1234

E-Mail karger@karger.ch

www.karger.com 


\section{Journat of Vascular Research}

\section{Committee}

Chairman: Daniel Henrion, Angers, France

\section{Local Organizing Committee:}

Georges Leftheriotis, University of Angers, France

Laurent Loufrani, University of Angers, France

Pierre Abraham, University of Angers, France

Bernard Enon, University of Angers, France

Jean-Louis Saumet, University of Angers, France

Gilles Berrut, University of Angers, France

\section{Scientific Committee:}

Christian Aalkjaer, University of Aarhus, Denmark

Ramaroson Andrianstitohaina, CNRS UMR7034, Strasbourg, France

Jo DeMey, College of Medicine, University of Maastricht, Netherland

Jean-Louis Freslon, Faculty of Pharmacy, Tours, France

David Harder, Medical College of Wisconsin, Milwaukee, Wisc., USA

Michael Hill, Medical School, RMIT University, Melbourne, Australia

Ismail Laher, College of Medicine, University of British Columbia, Vancouver, Canada

Akos Koller, College of Medicine, New York Medical College, Valhalla, N.Y., USA

Robert M. Lee, McMaster University, Hamilton, Canada

Bernard I. Levy, Med. School, INSERM U541, Paris, France

Michael Mulvany, University of Aarhus, Denmark

George Osol, College of Medicine, University of Vermont, Burlington, Vt., USA

Lucilla Poston, College of Medicine, University of London, UK

Vincent Richard, Med. School, INSERM, EMI 9920 Rouen, France

Ernesto Schiffrin, Clin. Res. Inst. of Montreal, University of Montreal, Canada

Ed vanBavel, Academic Medical Center, Amsterdam, Netherlands

\section{Invited Speakers:}

Axel Pries, Dept. of Physiology, Free University of Berlin, Berlin, Germany.

Gerald Meininger, Coll. of Medicine, Texas A\&M University, College Station, Tex., USA

Harry Struijker-Boudier, Cardiovascular Research Institut of Maastricht, Netherland

Ulrich Pohl, Physiology Institut, Ludwig-Maximilians-University Munich, Germany

Ernesto Schiffrin, Clinical Research Institute of Montreal, University of Montreal, Canada

Bernard I. Lévy, INSERM U541 \& Physiology Dept., Paris VII University, Paris, France

Eric Vicaut, Biophysic Dept., Paris VII University, Paris, France 


\section{Program}

June 20

19:00

June 21

8:00-09:00

9:00-10:15

10:45-13:00

14:00-15:00

15:00-17:00

17:00-18:30

19:00
Welcome reception: Hôtel d'Anjou

Opening lecture: Harry Struijker-Boudier (Maastricht, Netherland): Resistance artery research: future directions

Session 1: lonic control of vascular tone \& intercellular communication (Chair: I. Laher, Vancouver, Canada \& C. Aalkjaer, Aarhus, Denmark)

Coffee break (Poster room)

Session 2: Vascular biology: Smooth muscle (Chair: M. Hill, Melbourne, Australia \& G. Osol, Burlington, Vt., USA)

\section{Lunch}

Lecture: Axel Pries (Berlin, Germany): Regulation of vessel structure: Stimuli and reactions analyzed by mathematical simulation

Session 3: Pressure and flow-induced remodeling of resistance arteries (Chair: J. DeMey, Maastricht, Netherland \& M. Mulvany, Aarhus, Denmark)

Coffee and Poster session 1

\section{Cocktail}

at the 'Jean Lurçat tapestry museum' \& Dinner at the 'Greniers St Jean' 
Session 4: Vascular biology: Endothelium - part 1

(Chair: D. Harder, Milwaukee, Wisc., USA \& A. Koller, Valhalla, N.Y., USA)

Coffee break (Poster room)

11:00-12:30 Session 5: Vascular biology: Endothelium - part 2

(Chair: D. Harder, Milwaukee, Wisc., USA \& A. Koller, Valhalla, N.Y., USA)

Lunch

14:00-15:00

Lecture: Ulrich Pohl (Munich, Germany):

Calcium-(de)sensitising mechanisms in the control of microvascular tone

15:00-16:30 Workshop 1: Mice physiology, transgenic models. Transgenic models of human pathologies. New developments in genetic (DNA chips)

(Chair: Ed vanBavel, Amsterdam, Netherland \& R. Andriantsitohaina,

Strasbourg, France)

$16: 30-17: 30$

Coffee and Poster session 2

18:00 Gala dinner: Departure with buses for wine testing at Savennière 'Domaine de Closel' vineyard. Dinner at a panoramic restaurant along the Loire riverside.

Lecture: Gerald Meininger (College Station, Tex., USA): Integrin-extracellular matrix regulation of resistance artery function

9:00 - 10:15

Session 6: Reactive oxygen and inflammation.

(Chair: V. Richard, Rouen, France \& JL. Freslon, Tours, France)

Coffee break (Poster room)

$10: 45-12: 00$

Session 7: Hypertension (Chair: E. Schiffrin \& R. Lee)

12:00-12:45

Lecture: Eric Vicaut (Paris, France): Some aspects of oxidative stress-dependent mechanisms in muscle microcirculation

Lunch

$13: 30-14: 30$

Lecture: Bernard I. Lévy (Paris, France):

Role of the renin-angiotensin system in angiogenesis

14:30-16:30 Workshop 2: Human resistance arteries and pathology; Investigation of resistance arteries at the bedside. Information for clinical decisions (Chair: M. Mulvany, Aarhus, Denmark \& D. Henrion, Angers, France)

16:30-17:00 Coffee

End of the meeting 


\title{
Session 1: Ionic control of vascular tone \& Intercellular communication
}

\author{
Abtsract \#: $\quad 1-1$ \\ INTERACTION BETWEEN THE NA,K-PUMP AND THE ATP-SENSITIVE POTASSIUM CHANNEL IN RABBIT \\ CORONARY SMALL ARTERIES.
}

Matchkov VV; Glavind-Kristensen M; Brogaard Hansen V; Forman A; Nilsson H; Aalkjær C

Water and Salt Research Centre, Department of Physiology, University of Aarhus, Aarhus, DK-8000 Laboratory, Department of Obstetrics and Gynecology, Aarhus University Hospital, Aarhus, DK-8000

We tested the hypothesis that there is a functional interaction between the ATP-consuming Na,K-pump and the ATP-sensitive potassium channel in a rabbit small coronary artery. Isometric tension was recorded from isolated rabbit small coronary arteries and ATP-sensitive potassium current was recorded with conventional whole-cell patch-clamp technique in freshly isolated smooth muscle cells from these arteries. In arteries preconstricted with the thromboxane A2 analogue U46619 the ATP-sensitive potassium channel openers pinacidil and cromakalim induced concentration-dependent relaxations. Pinacidil-induced relaxation (max. relaxation $99 \pm 1 \%, n=7$ ) was not significantly affected by 1 microM of the ATP-sensitive potassium channel blocker glibenclamide (max. relaxation $97 \pm 1 \%, n=7$ ). However, the relaxation was partly inhibited in potassium-free media (max. relaxation $23 \pm 2 \%, n=6$ ) and by 1 microM of the $\mathrm{Na}, \mathrm{K}$-pump inhibitor ouabain (max. relaxation $56 \pm 6 \%, \mathrm{n}=8$ ). In the presence of both 1 microM glibenclamide and 1 microM ouabain a greater inhibition was seen (max. relaxation $38 \pm 4 \%, n=8$ ). In contrast, the cromakalim-induced relaxation (max. relaxation $96 \pm 1 \%, n=6$ ) was partly blocked by 1 microM glibenclamide (max. relaxation $35 \pm 11 \%, n=6$ ) and partly by 1 microM ouabain (max. relaxation $39 \pm 5 \%$, $n=3$ ) and when both drugs were present the inhibition increased (max. relaxation $4 \pm 4 \%, n=3$ ). Cromakalim and pinacidil induced a current across the smooth muscle cell membrane (current densities at $-60 \mathrm{mV}$ were $-6 \pm 1$ and $-6 \pm 2 A / F$, respectively, in the smooth muscle cells with the average capacity of $17 \pm 2 \mathrm{pF}$, $\mathrm{n}=6$ ), which reversed at the potassium equilibrium potential. The current was partly inhibited by 1 microM of glibenclamide (inhibition $24 \pm 3 \%, n=5$ and $41 \pm 11 \%, n=4$ of pinacidil- and cromakalim-induced current, respectively). Ouabain also inhibited this current (inhibition $26 \pm 6 \%, n=5$ and $42 \pm 2 \%, n=4$ of pinacidiland cromakalim-induced current, respectively). In the presence of both inhibitors a greater inhibition was seen (inhibition $54 \pm 4 \%, n=5$ and $83 \pm 10 \%, n=4$ of pinacidil- and cromakalim-induced current, respectively). However, ouabain had no effect $(0.5 \pm 2.6 \%, n=5$ and $0.3 \pm 5.9 \%, n=6$ of pinacidil- and cromakalim-induced current, respectively) on the current if $\mathrm{Na}+$ was omitted in the pipette (which will block the $\mathrm{Na}, \mathrm{K}$-pump) although glibenclamide was still effective $(35 \pm 7 \%, \mathrm{n}=5$ and $50 \pm 7 \%, \mathrm{n}=6$ of pinacidil- and cromakalim-induced current, respectively). The results strongly suggest a functional interaction between the Na,K-pump and the ATP-sensitive potassium channel which could be mediated through pump activity-dependent changes in the local environment for the channel (i.e. increased ATP/ADP ratio and/or decreased transmembrane potassium gradient) which partly prevent activation of the ATP-sensitive potassium current. 


\title{
Abtsract \#: $1-2$ \\ THE EFFECT OF THE ANION TRANSPORT BLOCKER DIDS ON INTRACELLULAR CHLORIDE AND VASOMOTION IN RAT MESENTERIC RESISTANCE ARTERIES.
}

\author{
Briggs DM; Nilsson H; Aalkjær C
}

Department of Physiology, Aarhus University, Ole Worms Allé 160, DK-8000, Aarhus C, Denmark.

\begin{abstract}
Many small arteries display a regular oscillation in vascular tone or diameter: a phenomenon known as vasomotion. Recently our group has proposed a model for vasomotion (1). Central to this model is the contribution of a calcium-activated ion channel, a candidate being the recently characterised calcium-activated cGMP-dependent chloride channel in mesenteric smooth muscle cells (2). The present work attempts to establish if a functional link exists between the chloride channel and vasomotion. All experiments were performed on rat isolated mesenteric resistance arteries (approx. $300 \mathrm{um}$ ). Arteries were mounted in a myograph for measurement of isometric force and intracellular chloride was determined in parallel experiments with $36 \mathrm{Cl}$. The intracellular chloride concentration $(n=8)$ was unaffected by $2 \mathrm{mM}$ DIDS and $200 \mathrm{uM}$ furosemide (to block $\mathrm{Cl} / \mathrm{HCO} 3$ exchange and $\mathrm{Na} / \mathrm{K} / 2 \mathrm{Cl}$ co-transport) and when applied to arteries in the myograph $1 \mathrm{mM}$ DIDS did not prevent noradrenaline-induced vasomotion $(\mathrm{n}=4)$. In further studies, vasomotion was abolished in arteries when the endothelium was removed but could be restored in the presence of $1 \mathrm{mM}$ DIDS $(n=12)$ with no significant difference in frequency or amplitude from controls. Oscillations in the presence of DIDS were blocked by $10 \mathrm{uM}$ ryanodine in all arteries tested $(n=6)$, as is the case with normal vasomotion. These results suggest that DIDS removes the cGMP-dependence from vasomotion, consistent with the hypothesis that a cGMP-dependent channel is involved in normal vasomotion. Surprisingly, we see another oscillatory system in the presence of DIDS suggesting that when anions are unable to contribute a cation channel contributes instead. 1. Peng $\mathrm{H}$, Matchkov V, Ivarsen A, Aalkjaer C, Nilsson H. (2001) Circ Res, 88:810-15. 2. Matchkov VV, Aalkjaer C, Nilsson H. (2004) J Gen Physiol, 123:121-34.
\end{abstract}


Abtsract \#: $1-3$

NA+/CA2+ (NCX) EXCHANGE IN MYOGENICALLY ACTIVE ARTERIOLES.

Raina H; Potocnik S; Kotecha N and Hill MA.

Microvascular Biology Group, School of Medical Sciences, RMIT University, Bundoora 3083, Melbourne Australia.

The arteriolar myogenic response plays a significant role in the setting of basal vascular tone and peripheral resistance. Arteriolar myogenic vasoconstriction is known to be associated with smooth muscle membrane depolarisation and opening of L-type voltage-gated Ca2+ channels. These events may be linked by the action of non-selective cation channels which would likely passage $\mathrm{Na}+$ under physiological conditions. In addition to modulating $\mathrm{Ca} 2+$ levels, this would be expected to activate $\mathrm{Na}+$ extrusion mechanisms. To determine a possible role for NCX in myogenically active isolated (rat) skeletal muscle arterioles, studies were conducted at several levels. Functional significance was determined by manipulation of extracellular $\mathrm{Na}+$ levels and inhibition of the exchanger using the relatively selective inhibitors KB-R7943 and SEA 0400. Changes in diameter, intracellular Ca2+ and membrane potential of cannulated vessels were measured in response to these manipulations. Identification of NCX protein was accomplished by western blotting using commercially available monoclonal and polyclonal antibodies raised against the NCX1 isoform. Total RNA was extracted from arterioles and real time PCR, using appropriate primers and sybr-green fluorescence, was performed to demonstrate levels of expression of mRNA for the NCX 1, 2 and 3 isoforms relative to the expression of 18sRNA. mRNA samples extracted from liver, heart, aorta, brain, and skeletal muscle were used for comparison. Experiments were conducted in intact arteriolar segments and in vessels that had the endothelium removed by perfusion of an air bubble. Reduction of extracellular $\mathrm{Na}+(137,100,75$ and $25 \mathrm{mM})$ caused significant steady-state vasoconstriction ( $P<0.5$, Regression Analysis), and an increase in Ca2+ $\mathrm{P}(\mathrm{P}<0.5$, Regression Analysis). Inhibition of NCX (30mM KB-R7943) in arterioles at physiological levels of extracellular $\mathrm{Na}+(137 \mathrm{mM})$ caused membrane hyperpolarization $(-34.2 \pm 0.9$ to $-42.8 \pm 1.9 \mathrm{mV} ; \mathrm{n}=4)$ and vasodilatation $(46.8 \pm 8.2$ to $131 \pm 10.2 \mathrm{~mm} ; \mathrm{n}=4)$. Vasomotor responses to $\mathrm{Na}+$ manipulation were not altered by endothelial cell removal. Western blotting showed the presence of a protein of approximately $70 \mathrm{kD}$ and $120 \mathrm{kD}$ consistent with studies in other tissues. PCR showed that arterioles expressed mRNA for all three isoforms with relative expression being NCX1 > NCX2 > NCX3; variation in expression pattern was, however, observed between tissues. The data are consistent with a functional role for the NCX in arterioles; however, complexity may exist due to the expression of multiple isoforms. 


\title{
Abtsract \#: $1-4$ \\ ON THE ORIGIN OF ARTERIAL CONTRACTION AND VASOMOTION: MODELING CALCIUM DYNAMICS, RECRUITMENT AND SYNCHRONIZATION OF COUPLED SMOOTH MUSCLE CELLS
}

\author{
Koenigsberger M; Sauser R; Lamboley M; Bény JL; Meister JJ;
}

Swiss Federal Institute of Technology Laboratory of Cell Biophysics CH - 1015 Lausanne Switzerland

\begin{abstract}
Many experimental studies have shown that arterial smooth muscle cells respond with cytosolic calcium rises to vasoconstrictor stimulation. Low vasoconstrictor concentrations give rise to asynchronous spikes in the calcium concentration in few cells (asynchronous flashing). With a greater vasoconstrictor concentration, the number of smooth muscle cells responding in this way increases (recruitment) and calcium oscillations may appear. These oscillations may eventually synchronize and generate arterial contraction and vasomotion. To the best of our knowledge, there is no theoretical study of these phenomena of asynchronous flashing, recruitment and synchronization. We propose a model describing the calcium dynamics of a two-dimensional population of smooth muscle cells coupled through their gap junctions. The effects of electrical, calcium and inositol 1,4,5-trisphosphate coupling are studied. We model the asynchronous flashing occurring at low vasoconstrictor concentration and explain why the number of recruited smooth muscle cells grows with increasing vasoconstrictor concentration. Moreover a weak gap junctional calcium permeability is shown to be necessary and sufficient to synchronize calcium oscillations. Electrical coupling can generate and desynchronize calcium oscillations. Inositol 1,4,5-trisphosphate diffusion does not play an important role to achieve synchronization. Our model is compared to in vitro experimental results obtained on strips of rat mesenteric arteries and is in agreement with them. We conclude that asynchronous flashing, recruitment and synchronization, thus arterial contraction and vasomotion, naturally emerge from a population of coupled smooth muscle cells.
\end{abstract}




\title{
Abtsract \#: $1-5$ \\ NITRIC OXIDE (NO•) AND THE NITROXYL ANION (NO-) CAUSE RELAXATION OF RAT MESENTERIC ARTERY BY DISTINCT MECHANISMS
}

\author{
Favaloro JL \& Kemp-Harper BK
}

Department of Pharmacology Monash University Clayton Vic 3800 Australia

Introduction: Nitric oxide is a key vasorelaxant molecule. Although the free radical form of nitric oxide (NO•) has been most widely studied, nitric oxide can exist in several different redox forms. In particular, the reduced form of nitric oxide, the nitroxyl anion (NO-), can be formed under physiological conditions from NO synthase. This raises the exciting possibility that NO- can also contribute to vasorelaxation, or that vasorelaxation responses previously attributed to $\mathrm{NO} \cdot$ may actually be mediated by NO-

Background and purpose: The aim of this study was to characterise and compare the relaxant and electrophysiological effects of $\mathrm{NO} \cdot$ and $\mathrm{NO}$ - in rat small mesenteric arteries. Materials and Methods: Rat small mesenteric arteries were mounted in a myograph and vascular tone and membrane potential were recorded. Cumulative concentration-response curves to NO • (NO gas) and the NO- donor, Angeli's Salt (AS), were constructed in the presence or absence of specific inhibitors. Results and Discussion: The relaxation response to $\mathrm{NO}$ - was accompanied by a hyperpolarisation, whilst relaxation to $\mathrm{NO} \cdot$ was independent of a change in membrane potential. In the presence of the NO scavenger $\mathrm{Cb}-\mathrm{PTIO}$ (200\&\#956;M), relaxation and hyperpolarisation responses to NO- were significantly inhibited by the NOscavenger L-cysteine (L-cys; 4mM), the SGC inhibitor ODQ (10 \&\#956;M), 30mM K+ and the KV channel inhibitor 4-aminopyridine (4-AP; $1 \mathrm{mM}$ ) (Relaxation (pEC50) control $7.133 \pm 0.163$ : L-cys $5.691 \pm 0.142$, ODQ $5.634 \pm 0.552, \mathrm{~K}+5.644 \pm 0.163,4-A P 4.835 \pm 0.428, \mathrm{n}=3-5, \mathrm{P}<0.05$, ANOVA; Hyperpolarisation to 10\&\#956;M AS (mV): control $23 \pm 4$, L-cys $15 \pm 1$, ODQ $6 \pm 1, K+0 \pm 0,4-A P 3 \pm 2, n=3-5, P<0.05$, ANOVA). In contrast, relaxation responses to $\mathrm{NO} \cdot$ were unaffected by L-cys, significantly inhibited by $\mathrm{Cb}-\mathrm{PTIO}$ and ODQ (Relaxation (pEC50) control $6.834 \pm 0.093$, L-cys $6.603 \pm 0.283 \mathrm{Cb}-\mathrm{PTIO} 5.832 \pm$ 0.230 , ODQ $5.924 \pm 0.410, n=3-5, P<0.05$, ANOVA). These data indicate that NO- causes relaxation via activation of sGC and KV channels, and suggest that cGMP activates the KV channel. In contrast, NO• mediates relaxation via SGC without involving $\mathrm{K}+$ channels. We conclude that $\mathrm{NO} \cdot$ and NO- cause vasorelaxation via distinct mechanisms. Given this, NO- donors may represent a novel class of nitrovasodilator agents which may be useful in treating cardiovascular disorders such as hypertension and angina. 


\title{
Abtsract \#: 1-6 \\ COMMUNICATION BETWEEN ENDOTHELIAL AND SMOOTH MUSCLE CELLS IN RAT MESENTERIC ARTERIES BY CHEMICAL COUPLING
}

\author{
Lamboley M 1, Pittet P 1, Koenigsberger K 1, Sauser R 1, Bény JL 2, Meister JJ 1.
}

1 Laboratory of Cell Biophysics, EPFL, CH-1015 LAUSANNE Switzerland 2 Department of Zoology and Animal Biology, University of Geneva, Quai E. Ansermet 30, CH-1211 GENEVA 4 Switzerland

Most evidence of gap junctional communication between endothelial (ECs) and smooth muscle cells (SMCs) concerns electrical coupling. A number of studies demonstrated an increase of intracellular calcium concentration ([Ca2+]i) in ECs as a consequence of $\mathrm{Ca} 2+$ increase in SMCs, but without being able to identify the diffusing molecule. Diffusion is a slow process in comparison to electrical spreading and chemical coupling would imply a measurable time lag between [Ca2+]i increase in SMCs and ECs, corresponding to the time necessary for ions to diffuse from cell to cell. Such a time delay has not been reported yet. Ca2+ or inositol trisphosphate (IP3) are susceptible to diffuse from SMCs to ECs to induce a $\mathrm{Ca} 2+$ increase in ECs. We have improved a method that allows studying simultaneously [Ca2+]i oscillations of individual SMCs and ECs in a rat mesenteric arterial strip, using confocal microscopy. [Ca2+]i was expressed as a fluorescence intensity variation obtained from Fura red AM and Fluo-4 AM fluorescence. SMCs were loaded with Fluo-4 and ECs with Fura red. Loaded strips were stimulated by increasing concentrations of the vasoconstrictors phenylephrine (PE) or $\mathrm{KCl}$ in presence or not of the gap junction uncoupler palmitoleic acid and of U-73122 to prevent hydrolysis of IP3. First, we demonstrate that $\mathrm{KCl}$ and $\mathrm{PE}$ act directly on SMCs and have no direct effect on ECs. Then, depending on vasoconstrictor concentration, we observed that [Ca2+]i increased in a small population of ECs 5 to $11 \mathrm{~s}$ after [Ca2+]i increased in all SMCs. This suggests a chemical communication from SMC to EC. In presence of $\mathrm{U}-73122$ and $\mathrm{KCl}$, all SMCs presented a global and synchronous [Ca2+]i increase, but no $\mathrm{Ca} 2+$ variations in $\mathrm{ECs}$ were detected. In presence of palmitoleic acid and $\mathrm{KCl}$ or $\mathrm{PE}$, the number of flashing ECs was dramatically reduced. Our observations demonstrate that [Ca2+]i increase in ECs appears with a given time lag after [Ca2+]i increase in SMCs. This time interval is a necessary condition to demonstrate a chemical coupling between SMCs and ECs. This coupling is responsible for the [Ca2+]i increase observed in ECs when SMCs are stimulated. In addition, our results suggest that IP3 and not $\mathrm{Ca} 2+$ is the molecule which diffuse from SMCs through heterocellular gap junctions and is secondarily responsible for ECs [Ca2+]i increase. 


\title{
Abtsract \#: $1-7$ \\ RHO KINASE MODULATES AN AGONIST-ACTIVATED CA ENTRY PATHWAY DISTINCT FROM THE CAPACITATIVE CA ENTRY IN RAT ISOLATED ARTERIES.
}

\author{
Morel N, Vandenberg G, Ghisdal P.
}

Université catholique de Louvain, Laboratoire de Pharmacologie, UCL 5410, Avenue Hippocrate, 54 B1200 Bruxelles, Belgium.

In vascular smooth muscle cells, contractile agonists activate a complex chain of events to increase cytosolic $\mathrm{Ca}$ concentration and contractile tension. The present study was aimed at characterising the $\mathrm{Ca}$ entry pathways activated by $\mathrm{G}$ protein coupled receptors. Ca signal measured by front surface fluorimetry in fura-2 loaded arteries, or membrane potential recorded with intracellular microelectrodes, were measured simultaneously with contraction, in rat aorta and mesenteric artery. In arteries bathed in Ca-free solution, response to noradrenaline consisted of a rapid but transient increase in cytosolic $\mathrm{Ca}$ and in tension. Re-addition of $\mathrm{Ca}$ into the Ca-free solution evoked a sustained increase in Ca signal, which was associated with an increase in tension. Noradrenaline-evoked $\mathrm{Ca}$ entry was inhibited by the voltage-dependent $\mathrm{Ca}$ channel blocker nimodipine by about $60 \%$. The nimodipine-resistant signal was sensitive to 2-APB and SKF 96365 and to low concentrations of Gd3+ (IC50 was $0.05 \mu \mathrm{M})$, and was inhibited by the Rho kinase inhibitor Y-27632 in a concentration-dependent manner (IC50 $0.5 \mu \mathrm{M}$ ). Y-27632 did not affect the Ca entry evoked by depolarisation in arteries bathed in high $\mathrm{KCl}$ solution. Similar effect was obtained with another inhibitor of Rho kinase, HA 1077. Rho kinase inactivation also inhibited the Ca signal evoked by direct activation of G proteins with AlF4-, which ruled out an interaction of the inhibitor with the alpha-adrenergic receptor. Disorganisation of the cytoskeleton with cytochalasin D, colchicine or nocodazole did not affect the Ca responses to noradrenaline, and did not prevent the inhibitory effect of Y-27632 on the Ca signal. Rho kinase inactivation inhibited the depolarisation evoked by noradrenaline. but did not affect the activation of phospholipase $C$ by noradrenaline. The release of intracellular Ca evoked either by caffeine or by IP3 were also unaffected by Rho kinase inhibition. Depletion of intracellular Ca stores by thapsigargin evoked a capacitative Ca entry, which was not associated with contraction. It was not affected by 2-APB, was sensitive to SKF 96365 and was blocked by Gd3+ in a concentration-dependent manner (IC50 $0.45 \mu \mathrm{M})$. The capacitative Ca entry was not affected by Rho kinase inhibitors. These results indicate that Rho kinase is involved in the activation of a phospholipase C-dependent Ca entry pathway in rat arteries, which contributes to the depolarisation and to the contraction evoked by agonists. This pathway is distinct from voltage-dependent Ca channels and from the capacitative $\mathrm{Ca}$ entry activated by store depletion. 
Abtspact \#: $1-8$

AN ANALYSIS OF THE DILATING RESPONSE OF MESENTERIC AND CEREBRAL RESISTANCE ARTERIES CAUSED BY TWO DIFFERENT CA2+-ANTAGONISTS.

\author{
Rasmussen CS; Mulvany MJ
}

Department of Pharmacology, Wilhelm Meyers Allé Bygn. 240, 8000 Århus C, Denmark

Recent clinical trials have shown that treatment with the dihyropyridine-derivates Ca2+-antagonist nimodipine given to Alzheimer's demented patients (L-Arrieta BJ, Cochrane Library, 2004, issue 2) is of some benefit, including delay in the onset of dementia. In the literature, nimodipine is referred to as being selective for cerebral arteries, but the evidence for this is not clear. We have therefore compared the dilator effect of nimodipine in rat cerebral and mesenteric small arteries, together with similar experiments using another dihyropyridine-derivate, nifedipine, as a control. Middle cerebral and mesenteric small arteries with a lumen dimension of approximately $200 \mu \mathrm{m}$ were dissected and mounted on a wire-myograph (Mulvany \& Halpern, 1977; Circ Res 41: 19-26. Buus et al. J Physiol 1998; 510.2: 577-590). Preconstriction was induced with serotonin (cerebral $1 \mu \mathrm{M}$, mesenteric $3 \mu \mathrm{M}$ ) or physiological saline solution substituted with $\mathrm{K}+[60 \mathrm{mM}]$. The Ca2+-antagonists were added at 10-9, 10-8, 10-7 and 10-6 M, 4 min per concentration. Wall tension at 3 min after each addition was expressed as percent of preconstriction. Not more than four vessels (two cerebral, two mesenteric) were taken from each animal. The results showed that both nifedipine and nimodipine had similar vasodilator effects on both cerebral and mesenteric arteries. However, some differences were noted. Nifedipine, $10 \mathrm{nM}$, relaxed cerebral arteries more than mesenteric vessels both with serotonin precontraction $(54.7 \%+/-4.7 \%, 68.0 \%+/-4.2 \%$, $\mathrm{n}=12, \mathrm{P}<0.05)$ and with $\mathrm{K}+$ precontraction $((45.3 \%+/-3.9 \%, 62.6 \%+/-6.5 \%, \mathrm{n}=12, \mathrm{P}<0.05)$. Nimodipine, $1 \mathrm{nM}$, relaxed cerebral arteries more than mesenteric vessels with $\mathrm{p} \mathrm{K}+$ precontraction $(73.3 \%$ $+/-3.2 \%, 86.3 \%+/-5.1 \%, \mathrm{n}=8-10, \mathrm{P}<0.05)$, but not with serotonin precontraction $(54.1 \%+/-5.0 \%, 62.5$ $+/-3.3 \%, n=10, P=0.18)$. We conclude that both nimodipine and nifedipine show some selectivity for cerebral vessels compared to mesenteric vessels, but that the selectivity is limited. Both drugs are slightly more potent against $\mathrm{K}+$ preconstrictions than against serotonin contractions, supporting their presumed inhibition of membrane L-type calcium channels. 


\begin{abstract}
Abtsract \#: $1-9$
KV3 VOLTAGE-DEPENDENT POTASSIUM CHANNEL SUBUNITS ARE EXPRESSED IN VASCULAR SMOOTH MUSCLE AND TRANSFORM A POTASSIUM CHANNEL BLOCKER INTO A STIMULATOR
\end{abstract}

\author{
Schubert R, Bittorf T, Ulfig N, Gagov H
}

University Rostock, Institute of Physiology, PSF 100888, 18055 Rostock, Germany

Introduction: Voltage-dependent potassium channels (Kv channels) play an important role in the regulation of the contractility of small arteries. The Kv channel family consists of a large number of subunits. However, only for a few of them a functional role has been described in small arteries. Purpose: The aim of the study was to test the role of Kv3 channel subunits in smooth muscle cells from small cerebral and skeletal muscle arteries from Wistar rats. Methods: The existence and localization of Kv3 channel subunits was explored with Western-blotting and immunohistochemistry. The characteristics of Kv currents were studied with the patch-clamp technique. Results and Discussion: Kv3 channel subunits were detected in smooth muscle cells from both small arteries investigated. The Kv current of these cells showed biophysical and pharmacological properties similar to recently described Kv currents from rat and mouse small arteries. The specific blocker of homomultimeric Kv3 channels, BDS-I, affected these currents. The BDS-I-sensitive current was larger than the current remaining after eliminating Kv1 currents with the specific Kv1 channel inhibitor correolide and was unaffected by $1 \mathrm{mM}$ TEA, known to block homomultimeric Kv3 channels completely. Therefore, Kv3 subunits seem to exist as heteromultimeric channels together with non-Kv3 subunits. The application of $10 \mu \mathrm{M}$ BDS-I induced a slowing of the time course of activation and a shift of the voltage-dependence of activation from $-7.7+/-2.2 \mathrm{mV}$ to $5.1+/-2.7$ $\mathrm{mV}$ without affecting the time course of deactivation. In addition, BDS-I induced a slowing of the time course of inactivation, an acceleration of the recovery from inactivation and a shift of the voltage-dependence of steady-state inactivation from $-33.3+/-1.3$ to $-16.9+/-1.9 \mathrm{mV}$. In order to understand the effect of BDS-I taking into account the simultaneous action on activation and inactivation, Kv currents were measured from a holding potential of $-40 \mathrm{mV}$, the resting membrane potential of the vessels studied. BDS-I at $10 \mu \mathrm{M}$ increased the outward current 2.4 times at $+30 \mathrm{mV}$. This effect was completely inhibited by correolide. In conclusion, the data show that BDS-I increases the Kv current at physiological holding potentials due to a considerable alteration of the voltage-dependence of inactivation. In addition, the unexpected BDS-I-induced activation of the Kv current suggests that Kv3 subunits form heteromultimeric channels together with non-Kv3 subunits in rat small artery smooth muscle cells. 


\section{Abtsract \#: $1-10$ \\ PERIVASCULAR ADIPOSE TISSUE RELEASES A VASODILATOR IN HUMAN INTERNAL THORACIC ARTERY}

Gao YJ.(1); 1 Zeng ZH.; 2 Teoh K.; 2 Abouzahr L.; 2 Cybulsky I.; 2 Lamy A.; 2 Semelhago L.; 1 Lee R.M.K.W.

1Department of Anaesthesia, 2Cardiovascular Surgery, McMaster University, 1200 Main Street West, Hamilton, Ontario, Canada L8N $3 Z 5$

Recent studies have shown that in addition to its passive role as a storage tissue for excess energy, adipose tissue is also very active metabolically, producing a number of biologically active substances. In the vascular system, virtually all the systemic blood vessels are surrounded by perivascular adipose tissue (PVAT). Two studies have reported that PVAT from rat secretes a substance which can dilate the aorta. The purpose of the present study is to examine if this vasodilator is also present in the PVAT of human arteries. Remnants of human internal thoracic arteries which are discarded during coronary artery bypass grafting were used. The presence of PVAT attenuated the contraction to phenylephrine and U 46619. To assess the direct effects of PVAT in inhibiting vasoconstriction, we did a bioassay experiment by transferring the bathing solution incubated with a PVAT-intact vessel (donor) to a vessel without PVAT (recipient) under the same precontracted condition. This transfer induced a significant relaxation in the recipient artery, while the same transfer procedure between PVAT-deprived arteries did not. Transfer of bathing solution with isolated PVAT induced a similar relaxation response as the bathing solution from PVAT-intact vessel. The relaxation was prevented by $\mathrm{KCl}(60 \mathrm{mM})$. Calcium-dependent potassium channel blocker, TEA (1 mM) and iberiotoxin (10-7 M) completely blocked the relaxation, while voltage-dependent potassium channel blocker 4-aminopyridine $(1 \mathrm{mM})$ and ATP-dependent potassium channel blocker glibenclamide (10 uM) did not. We suggest that human internal thoracic arteries PVAT releases a transferable relaxing factor which acts through the activation of Kca channels. 
Abtsract \#: $1-11$

A NOVEL PROTEIN KINASE G MODULATED, CALCIUM-ACTIVATED CHLORIDE CHANNEL IN SMOOTH MUSCLE CELLS.

\author{
Matchkov VV; Aalkjaer C; Nilsson H
}

Department of Physiology, University of Aarhus, Ole Worms Alle 160, DK-8000 Aarhus C, Denmark

We have previously reported on a unique cGMP-dependent calcium-activated chloride current in smooth muscle cells (SMCs) from mesenteric resistance arteries, and suggested this to be of importance in synchronizing smooth muscle contraction. Here we have characterized this novel current with respect to ion selectivity, protein kinase G (PKG) regulation and tissue distribution. Using conventional patch-clamp technique, whole-cell currents were evoked by elevation of intracellular calcium in rat freshly isolated SMCs from different vasculature: aorta, pulmonary, tail, renal, superior mesenteric and mesenteric small artery and iliac vein. The novel current was found to be a calcium-activated chloride current with an absolute requirement for cyclic GMP (EC50 6.4 microM). The current could be activated by dialyzing the cell by the cGMP-independent, constitutively active subunit of PKG. The current could be also activated by ATP-gamma-S in the absence of cGMP, and in this condition cGMP had no further effect. Current activation was blocked by the protein kinase $\mathrm{G}$ antagonist Rp-8-Br-PET-cGMP or with a peptide inhibitor of PKG, or with the non-hydrolysable ATP analog AMP-PNP. Under bi-ionic conditions, the anion permeability sequence of the channel was $\mathrm{SCN}>\mathrm{Br}>\mathrm{I}>\mathrm{Cl}>$ acetate $>\mathrm{F}>>$ aspartate, but the conductance sequence was I $>\mathrm{Br}>\mathrm{Cl}>$ acetate $>\mathrm{F}>$ aspartate $=\mathrm{SCN}$. The current showed no rectification or time-dependence. It had a high sensitivity to zinc and nickel, but a low sensitivity to niflumic acid, DIDS and IAA-94. The properties of the cGMP-sensitive current differed from those of the classical calcium-activated chloride current, which was cGMP-independent, exhibited a high sensitivity to inhibition by niflumic acid, was unaffected by zinc ions, and showed outward current rectification. Under conditions of high calcium in the patch pipette solution, both currents were found to coexist in SMCs from superior mesenteric artery and mesenteric small artery. Only the cGMP-independent current was found in pulmonary artery SMCs and this was dominant in tail artery SMCs. The cGMP-sensitive current was dominating in SMCs from aorta, renal artery and iliac vein. Thus rat vascular SMCs shows two types of calcium-activated chloride current, one of which is regulated by cGMP via PKG. These currents are distributed differently in different vascular tissues. 


\title{
Abtsract \#: $1-12$ \\ INTRACELLULAR RHYTHMIC CALCIUM OSCILLATIONS IN VASCULAR SMOOTH MUSCLE CELLS ARE PROPRIETY OF COUPLED CELLS
}

\author{
Fanchaoui M; Serir K; Bytchkov R; Bény J-L.
}

Department of zoology and animal biology, Geneva university, Quai E.-Ansermet 30, 1211 Geneva 4, Switzerland

Introduction: Vasomotion has been known as spontaneous variations in the muscular tone in small, resistance-type arteries and arterioles. Rhythmic oscillations of cytosolic free calcium are responsible for vasomotion. However, the mechanism behind this rhythmic oscillation has not yet been fully understood. Models of calcium oscillations are based on the interaction between two oscillators: an endoplasmic reticulum and a cell membrane one. Background and purpose: These models envisage the tissue as composed of a homogeneous population of synchronized smooth muscle cells. In this concept, the tissue function as one cell. But, the inhibition of cell-to-cell communication by gap junctions has shown to inhibit vasomotion. Therefore we tested the hypothesis that rhythmic calcium oscillation could result from cell-to cell coupling. Results and discussion: On acutely dispersed smooth muscle cells from mouse aorta, exogenous ATP $(250 \mu \mathrm{M})$ elicited [Ca2+]i transient responses, sustained non inactivating [Ca2+]i raise, periodic $[\mathrm{Ca} 2+] \mathrm{i}$ oscillations and non periodic repetitive $[\mathrm{Ca} 2+] \mathrm{i}$ transients. Simultaneous measurement of $[\mathrm{Ca} 2+] \mathrm{i}$ changes and ion currents revealed correlation between $[\mathrm{Ca} 2+] \mathrm{i}$ spikes and ion current peaks. One type of Ca2+-dependent current spikes was inward (58\%), while second was outward $(42 \%)$ at holding potential $-30 \mathrm{mV}$. Two observations demonstrate the implication of gap junctions in calcium oscillations. Firstly, quantity of smooth muscle cells in the preparation that responded to ATP with periodical [Ca2+]i oscillations depended from the isolated cells density on the cover slip. ATP elicited bursts of [Ca2+]i spikes in $66 \pm 7 \%$ of cells in dense and in $33 \pm 8.5 \%$ of cells in non dense preparations. The number of cells that responded to ATP with bursts [Ca2+]i spikes decreased from $55 \pm 5 \%(n=84)$ in control to $14 \pm 3 \%$ $(n=141)$ after 10 minutes pretreatment of dense preparations with carbenoxolone a cell-to-cell coupling inhibitor. Secondly, in the same way, ATP elicited bursts of calcium-dependent currents spikes in $76 \%$ of cells regrouped in small clusters composed from two to five cells but only in $9 \%$ of isolated cells. Application of carbenoxolone $(100 \mu \mathrm{M})$ abruptly inhibited ATP $(250 \mu \mathrm{M})$ elicited Ca2+-dependent bursts of spiking currents. In these experiments, measurement of cell capacitance allowed to correlate cell-to-cell coupling with current oscillations. Taken together these results suggest that the probability of ATP (250 $\mu \mathrm{M})$ triggered cytosolic [Ca2+]i oscillations accompanied with $\mathrm{K}+$ and $\mathrm{Cl}$ - currents oscillations is not a propriety of isolated smooth muscle cells but emerge only when at least two cells are coupled. By introducing cell-to-cell coupling in model of calcium control, we successfully modeled this behavior. 


\title{
Abtsract \#: $1-13$ \\ VASOPRESSIN ACTIVATES A NOVEL LANTHANIDE-RESISTANT NON-SELECTIVE CATION CURRENT IN A7R5 VASCULAR SMOOTH MUSCLE CELLS.
}

\author{
Brueggemann LI; Byron KL
}

Loyola University Chicago, Stritch School of Medicine, Maywood, IL 60153, USA

\begin{abstract}
Vasoconstrictor hormones have been reported to stimulate action potential firing in arterial smooth muscle cells (SMC), although the ionic mechanisms underlying this effect are not well understood. Excitation of SMC may depend on activation of non-selective cation currents (NSCC). [Arg8]-vasopressin (AVP) triggers action potential firing in the A7r5 rat aortic SMC line. Using whole-cell patch-clamp techniques we detected a novel lanthanide-resistant NSCC (I-LRC) in A7r5 cells. This outwardly rectifying current was transiently activated by AVP in the presence of 100 micromolar Gd3+ or La3+, which blocked all other NSCC. The EC50 for activation of I-LRC by AVP was $1.0 \pm 0.1 \mathrm{nM}$ for outward and $5.0 \pm 1.9 \mathrm{nM}$ for inward current components. ILRC was carried predominantly by monovalent cations and required external organic anions and lanthanides or $\mathrm{Ca} 2+$ for activation. I-LRC was not activated by the diacylglycerol analog, 1-oleoyl-2-acetyl-sn-glycerol (OAG, 10 micromolar) or by depletion of intracellular Ca2+ stores. Passive empting of $\mathrm{Ca} 2+$ stores with $10 \mathrm{mM}$ BAPTA did not activate I-LRC but slowed activation and inactivation of current in the presence of AVP. I-LRC was directly activated or enhanced by flufenamic acid. This novel receptor-activated current may be involved in the AVP-induced membrane depolarization that precedes action potential firing.
\end{abstract}




\title{
Session 2: Vascular biology: Smooth muscle
}

\author{
Abtsract \#: $2-1$ \\ AGEING DIFERENTELY AFFECT INOS AND COX-2 PATHWAYS IN MESENTERIC RESISTANCE \\ ARTERIES.
}

Vila E1, Salaices M2 and Briones AM1.

1Dpt. Farmacologia,Terapeutica i Toxicologia, Facultat de Medicina, Universitat Autónoma de Barcelona. Bellaterra, 08193, Barcelona and 2Dpt. Farmacología y Terapéutica. Facultad de Medicina. Universidad Autónoma de Madrid, C/Arzobispo Morcillo 28029, Mad

Introduction: Production and/or plasma concentration of cytokines increase in both animals and elderly humans (Foster et al., Am J Physiol 262, R211-5, 1992; Wei et al., Life Sci 51, 1953-6, 1992). Background and purposes: Ageing changes iNOS mRNA and COX-2 protein expression in rat tail artery and aorta respectively (Tabernero et al., Br J Pharmacol, 131, 1227-35, 2000; Boulanger et al., Br J Pharmacol, $131,804-10,2000)$. The purpose of the present study was to analyse the influence of ageing in the production of inflammatory mediators induced by IL-1ß in rat mesenteric resistance arteries. Material and Methods: Second and third branches of rat mesenteric arteries from 3 (young) and 22 (old) months-old male Sprague-Dawley rats were incubated in culture medium either with or without IL-1ß\&\#61472;\&\#61480; (10 ng/ml, 14 h). The following parameters were analysed: i) Nitrite (Griess reaction) and prostanoids (enzyme immunoassay) production; ii) inducible nitric oxide synthase (iNOS) and cyclooxygenase (COX-2) expression by Western blot and immunohistochemistry and iii) contractile responses to phenylephrine were also determined in 3rd branches of mesenteric arteries in a wire myograph. Results: Nitrite production and iNOS expression were significantly enhanced by IL-1ß incubation in arteries from both age groups. The relative increase in both parameters was greater in young than in old animals. COX-2 protein expression but not TXB2 and PGF2 $\alpha$; levels were increased by IL-1ß in both age groups. The level of PGI2 was increased to a similar extent in young and old animals. However, vessels from aged animals showed higher levels of contractile prostanoids compared to young animals. iNOS and COX-2 were localised in endothelial, smooth muscle and adventitial cells. Phenylephrine induced contraction after IL-1ß was higher in arteries from old than young rats. Discussion: IL-1ß induced an inflammatory response in mesenteric resistance arteries associated to increased iNOS and COX-2 expression. The higher increase on nitrite production from iNOS in young rats together with a greater contractile prostanoids production in old rats, could be responsible of the observed decrease on phenylephrine mediated contraction in young compared to old animals after IL-1ß treatment. (Supported by DGCYT (2003-01001) and Fundación Salud 2000) 


\section{Abtsract \#: $2-2$ \\ CHANGES IN THE FUNCTION OF PERIVASCULAR ADIPOSE TISSUE DUE TO PRENATAL EXPOSURE TO NICOTINE}

Gao YJ.(1); 2 Holloway AC.; 1 Zeng ZH.; 2 Lim GE.; 3 Petrik JJ.; 2 Foster WG.; 1 Lee RMKW.

1Department of Anaesthesia and 2Obstetrics and Gynaecology, McMaster University, Hamilton, Ontario, Canada; 3Department of Biomedical Sciences, University of Guelph, Ontario, Canada

Prenatal exposure to nicotine has been shown to result in low birth weight and subsequent growth of the offsprings. We have found that prenatal exposure to nitocine increased body weight and visceral fat pad weight in adult Wistar rats. Since some recent studies have shown that perivascular adipose tissue (PVAT) secretes a factor which relaxes the blood vessels of rats, the aim of the present study was to determine if the modulator function on vessel contraction by PVAT was altered in these overweight rats. Body weight of the rats exposed to nicotine began to increase at 9 weeks of age as compared with control until 26 weeks when the study was terminated. Fat pads in the mesenteric, epididymal and perirenal tissues were significantly heavier than those in the control rats. In the thoracic aorta and mesenteric arteries, the amount of PVAT was significantly higher in the offspring from nicotine-exposed dams than those from saline-exposed (control) dams. In the aortic rings from control rats, the presence of an intact PVAT significantly attenuated the contraction to phenylephrine (PE), while in the aorta of the rats from nicotine-exposed dams the inhibition of contraction to PE by PVAT was not observed. The contraction of rings without PVAT to PE was not different between offspring from saline and nicotine-exposed dams. Transfer of solution incubated with PVAT-intact aorta to PVAT-free aorta induced a marked relaxation response in the rats from saline-exposed dam, but this relaxation response was significantly impaired in the rats from nicotine-exposed dams. These results showed that prenatal exposure to nicotine increased PVAT deposition, along with an increase in body weight and visceral fat pads. The inhibitory action of PVAT on PE-induced contraction was significantly reduced in rats exposed to nicotine. This reduction may reflect a reduced production/release of vasorelaxant substance from the PVAT in rats exposed to nicotine prenatally. 
Abtsract \#: $2-3$

MECHANISM OF RHOA/RHO KINASE IN STRETCH-INDUCED MYOGENIC TONE

Caroline Dubroca, B.Sc., *Laurent Loufrani, Ph.D., Bernard I. Lévy, M.D., Ph.D., *Daniel Henrion, Pharm.D., Ph.D.,

Inserm U541, 41 Bvd de la chapelle 75010 Paris

Myogenic tone, a fundamental property of resistance arteries and veins, is a maintained vasoconstriction of the microvascular smooth muscle due to pressure. Myogenic tone is a key determinant of local blood flow regulation. The aim of this study was to evaluate the pathways involved in myogenic tone. The role of the RhoA/Rho kinase, p38 MAP kinase and HSP27 in myogenic tone was investigated in the rabbit facial vein (RFV) which has a unique feature. Myogenic tone in the RFV is temperature-sensitive. Thus, myogenic tone in stretched RFV $\left(39^{\circ} \mathrm{C}\right)$ can be compared to stretched RFV without tone $\left(33^{\circ} \mathrm{C}\right)$, which is not the case in arteries studied with or without pressure. Indeed, stretch may activate multiple pathways not necessarily involved in myogenic tone. Isolated RFV segments were mounted in organ baths and stretched. Temperature was set at $33^{\circ} \mathrm{C}$ or $39^{\circ} \mathrm{C}$. The Rho kinase inhibitor Y27632 decreased stretch-induced myogenic tone by $93.1 \pm 4.9 \%$ and the p38 MAP kinase inhibitor SB203580 by $36.5 \pm 8.1 \%$. Stretch $\left(39^{\circ} \mathrm{C}\right)$ induced myogenic tone, the translocation of RhoA to the plasma membrane, an increase in p38 phosphorylation $\left(131,0 \pm 12,5 \%\right.$ at $39^{\circ} \mathrm{C}$ versus $100 \%$ at $33^{\circ} \mathrm{C}$ ) and $\mathrm{HSP} 27$ phosphorylation (196.1 $\pm 10,3 \%$ versus $100 \%)$, compared to RFV segments stretched at $33^{\circ} \mathrm{C}$. Phosphorylation of p38 was blocked by Y27632 and that of Hsp27 was by SB203580 and Y27632. High potassium-induced contraction was not affected by SB203580 or Y27632. Thus, myogenic tone was fully dependent of RhoA/Rho kinase activation and partly dependent of p38 and Hsp27 activation. Phosphorylation of p38 and Hsp27 was dependent of RhoA/Rho kinase activation. 


\title{
Abtsract \#: $2-4$ \\ INHIBITION OF PROLIFERATION OF SMOOTH MUSCLE CELLS FROM HUMAN ATHEROSCLEROTIC ARTERIES BY BRIEF EXPOSURE TO A RAS FARNESYL TRANSFERASE INHIBITOR, FTP III
}

\author{
Coats P; Wainwright CL; Gurney AM; Pyne S; Wadsworth RM.
}

University of Strathclyde, Dept Physiology and Pharmacology, 27 Taylor Street Glasgow G4 ONR, UK

\begin{abstract}
Vascular smooth muscle cell (VSMC) proliferation is a predominant component of primary atherosclerotic plaque formation and restenosis following balloon angioplasty. Activation of farnesylated p21ras and p42/p44 mitogen-activated protein kinase (p42/p44 MAPK) following balloon angioplasty are well established in models of arterial restenosis. Indeed, we have previously shown that chronic (18 hours) treatment of VSMC with the farnesyl transferase inhibitor, FTP III, inhibits proliferation. However, we also established that brief (15 minutes) in-vivo delivery of FTP III inhibits restenosis following balloon angioplasty1. The aim of the present study was to further examine the mechanism underlying this effect. Arteries were isolated from patients undergoing amputation for peripheral vascular disease. VSMC were derived from explants from cultured arterial rings. FTP III (1-50\&\#61549;M, 18 hr) inhibited serum-stimulated VSMC proliferation as measured by $[3 \mathrm{H}]$ thymidine incorporation (IC50,

10ะ1\&\#61549;M; and by $72 \pm 6 \%$ using 25\&\#61549;M FTP III). This correlated with subsequent inhibition of platelet-derived growth factor (PDGF)-stimulated p42/p44 MAPK activation as measured by Western blotting. Similarly, acute (15 minute) exposure of VSMC to FTP III reduced smooth muscle cell proliferation (by $45 \pm 5 \%$ using 25\&\#61549;M FTP III). The inhibitory effect was not significantly different between 15 minutes and 30,60 or 120 minutes exposure. However, the inhibition of VSMC proliferation following acute exposure to FTP III was not associated with subsequent inhibition of PDGF-stimulated p42/p44 MAPK activation. These data suggest that (i) the acute and chronic treatments of VSMC with FTP III may inhibit subsequent DNA synthesis through differing mechanisms and (ii) in the clinical setting, short-term local delivery of FTP III may have the potential to limit neo-intimal formation that occurs at the sight of arterial anastomoses following a revascularisation procedure and that associated with balloon injury. 1. Work et al (2001) Circ. 104:1538-1543
\end{abstract}




\title{
Abtsract \#: $2-5$ \\ IDENTIFICATION OF THE ENDOPLASMIC RETICULUM IN PRESSURISED HUMAN SMALL ARTERIES BY ELECTRON MICROSCOPY
}

\author{
Sweeney M1; Jones CJP1; Baker PN1; Taggart MJ1,2
}

1Maternal and Fetal Health Research Centre, 1,2Smooth Muscle Physiology Group, Cardiovascular Research, University of Manchester, St. Mary's Hospital, Whitworth Park, M13 0JH, United Kingdom

Transient elevations of cytosolic $\mathrm{Ca} 2+$ that are either localised (sparks) or that propagate along the length of a vascular smooth muscle cell (waves) are dependent upon $\mathrm{Ca} 2+$ release from the intracellular store of endoplasmic reticulum (ER). These dynamic events have been demonstrated to be important in regulating tone in a wide variety of animal vascular smooth muscle cell (VSMC) preparations. However, these phenomena have not yet been reported in intact human arteries. The aim of this study was to examine the ultrastructure of VSMC from human arteries in order to determine their potential for production of $\mathrm{Ca} 2+$ sparks and waves. Small $(140-350 \mathrm{~mm}$ intralumenal diameter) omental arteries were isolated from pregnant women at time of elective Caesarean section, following written informed consent, and mounted on a pressure myograph at $50 \mathrm{mmHg}$. Following equilibration, arteries (resting or contracted with $10-6 \mathrm{M} \cup 46619$ ) were fixed in $2.5 \%$ glutaraldehyde, $0.1 \mathrm{M}$ sodium cacodylate with 3 or $50 \mathrm{mM} \mathrm{CaCl} 2$ and postfixed in osmium ferricyanide to enable ER identification (Nixon et al., 1994, J. Muscle Res. Cell Motil. 15:682-700). Resin-embedded ultrathin (<100nm) longitudinal and transverse sections were examined electron microscopically. The vascular wall consisted of mainly longitudinal smooth muscle cells 2-4 layers thick with large intercellular spaces containing loose connective tissue. Some intercellular connections were observed. Within the VSMC, a prominent central localisation of ER together with mitochondria, the Golgi apparatus and nucleus was evident. This centrally located ER appeared continuous with the nuclear membrane. Enveloping these structures were tightly packed arrays of myofilaments orientated in parallel to the longitudinal axis of the cells. It is of note that the endothelial cells of the vessel wall also exhibited an ER structure extending along the length of the cell. The ER of VSMC was also located near the plasma membrane in close association with abundant caveolae, often completely surrounding them. On occasion, this peripheral ER was also orientated perpendicular to the plasma membrane, extending deeper into the cytoplasm. Taken together, these observations suggest that the ER in human VSMC may exist as a continuous network similar to that reported in animal VSMC. In pressurised human arteries, therefore, the ER ultrastructure in VSMC is consistent with that required for the production of $\mathrm{Ca} 2+$ sparks and waves, which may be an important determinant of the regulation of tone in these arteries. Supported by Tommy's, the Baby Charity. 


\title{
Abtsrat \#: $2-6$ \\ IMMUNOHISTOCHEMICAL CHARACTERIZATION OF $\alpha$;1-ADRENOCEPTORS IN RAT FEMORAL RESISTANCE ARTERIES.
}

\author{
Zacharia J*,Hillier C*,Daly CJ+, McGrath JC+ \& MacDonald A*.
}

*Department of Biological and Biomedical Sciences, Glasgow Caledonian University, Glasgow. +IBLS, University of Glasgow, Glasgow UK.

Previously we have shown that $\alpha ; 1 \mathrm{~A}$-adrenoceptors (ARs) are the predominant subtype involved in vasoconstriction in rat femoral resistance arteries with a contribution of $\alpha ; 1 D-A R$ at low frequencies of stimulation (Zacharia et al., 2004). In the present study we characterized $\alpha ; 1-A R s$ in rat femoral resistance arteries using primary antibodies specific to rat and mouse $\alpha ; 1 \mathrm{~A}-, \alpha ; 1 \mathrm{~B}-$ and

$\alpha ; 1 D-A R s$ (isotype, goat IgG, Santa Cruz biotechnologies). Third order branches of rat femoral artery $($ c. $220 \mu \mathrm{m})$ were dissected out from male Wistar rats $(200-250 \mathrm{~g}, 10-13$ weeks old). The arteries were formalin fixed, processed and embedded in wax using standard techniques. The sections were subjected to antigen retrieval using the microwave/pressure method and blocked with $20 \%$ donkey serum to reduce non-specific antibody staining. Sections were then incubated with specific $\alpha ; 1$-adrenoceptor subtype primary antibodies (dilution 1:25) for $12 \mathrm{hrs}$ at $4 \mathrm{oC}$. The sections were washed in phosphate buffer saline and receptors were localized using a fluorescein isothiocyanate (FITC) tagged donkey anti-goat secondary antibody. The sections were viewed under a fluorescent microscope for specific staining. For $\alpha ; 1 \mathrm{~A}-\mathrm{ARs}$ the positive control used was rat vas deferens. For $\alpha ; 1 \mathrm{~B}-\mathrm{ARs}$ the positive control was rat liver and a negative control was $\alpha ; 1 \mathrm{~B}-\mathrm{AR}$ knockout (KO) mouse liver. For $\alpha$;1D-ARs the positive control was rat aorta and a negative control was $\alpha ; 1 \mathrm{D}-\mathrm{AR}$ KO mouse aorta. The positive and negative controls were processed in the similar manner to rat resistance arterial sections. A negative control without primary antibody was also used to identify background florescence. Staining of femoral resistance arteries with the $\alpha ; 1 A-A R$ antibody $(n=6)$ showed a strong immunoreactivity indicating presence of $\alpha ; 1 A-A R s$ throughout the walls of the artery. Staining with $\alpha ; 1 D-A R$ antibody $(n=5)$ also produced immunoreactivity throughout the walls of the artery (with a lesser intensity than $\alpha ; 1 \mathrm{~A}-\mathrm{AR}$ antibody) indicating presence of $\alpha ; 1 \mathrm{D}-\mathrm{ARs}$. Staining with $\alpha ; 1 \mathrm{~B}-\mathrm{AR}$ antibody $(\mathrm{n}=4)$ showed no apparent immuno-reactivity. Antibody specificity was shown using positive, negative and KO controls. In conclusion, immunohistochemical characterization of $\alpha ; 1-A R s$ has shown the presence of both $\alpha ; 1 \mathrm{~A}$ - and $\alpha ; 1 \mathrm{D}$-ARs in rat femoral resistance arteries consistent with the presence of these subtypes in functional studies. The lack of staining with $\alpha ; 1 \mathrm{~B}-\mathrm{AR}$ antibody is also in agreement with the lack of involvement of this subtype in responses to exogenous and endogenous noradrenaline. Zacharia. J et al., 2004. Br. J. Pharmacol, 141, 915-924. 


\title{
Abtsract \#: $2-7$ \\ THE ROLES OF PROTEIN KINASE C ISOFORMS, RHO-KINASE, AND TYROSINE KINASE IN THE PATHOPHYSIOLOGY OF VASOSPASM AFTER SUBARACHNOID HEMORRHAGE
}

\author{
Nishizawa S; Koide M; Yamaguchi M; Obara K*; Nakayama K*
}

Department of Neurosurgery, Hamamatsu University School of Medicine and Department of Cellular and Molecular Pharmacology, Graduate School of Pharmaceutical Sciences, University of Shizuoka, 1-20-1 Handayama Hamamatsu, 431-3192 Japan

[Introduction and background] The mechanism of vasospasm after subarachnoid hemorrhage (SAH) remains unclear. We have extensively examined the roles of protein kinase $C$ (PKC) and PKC-isoforms in the mechanism of vasospasm, and identified that four PKC-isoforms are present in a canine basilar artery, PKC-alpha, delta, zeta, and eta. Among these isoforms, PKC-delta initiates vasospasm and PKC-alpha maintains the prolonged cerebral arterial contraction after SAH in in-situ treatment study using PKC inhibitors. [Purposes] Based on these experimental results, we further investigated the interrelation of PKC-isoforms, phosphorylation of myosin light chain, rho-kinase, and tyrosine kinase in the mechanism of vaospasm after SAH. [Materials and Methods] "Two-hemorrhage" canine model was used to conduct experimental SAH and vasospasm, and a basilar artery was used for following experiments. [Results] Phosphorylation of myosin light chain was significantly enhanced during vasospasm and continued more than two weeks. Under significant angiographic inhibition of vasospasm using PKC inhibitors, enhanced myosin light chain phosphorylation continued. In-situ treatment study using "two-hemorrhage" canine model, Y-27632, a specific inhibitor of rho-kinase, inhibited the phosphorylation of myosin light chain and PKC-delta activity, but not PKC-alpha. Angiographic study showed that Y-27632 inhibited only the initial phase of vasospasm, but not maintenance phase even under the significant inhibition of myosin light chain phosphorylation. In the "two-hemorrhage" canine model, total PKC activity, the translocation of PKC-delta and -alpha returned to the control levels within two weeks. However, angiographic vasospasm continued for two weeks, thereafter, returned to the normal arterial caliber. From one week after the hemorrhage, total activity of tyrosine kinase increased, and enhanced activity of tyrosine kinase continued for two weeks. In an isometric tension study, enhanced tone of canine basilar artery excised two week after SAH was significantly inhibited by genistein, a tyrosine kinase inhibitor. [Discussion] These results indicate that the initiation of vasospasm depends on the PKC-delta activity. The rho-kinase modulates the initiation of vasospasm through PKC-delta activity. PKC-alpha contributes the maintenance of vaospasm until one week after SAH. Because Y-27632 did not inhibit PKC-alpha activity, rho-kinase and $\mathrm{PKC}$-alpha are functionally independent. However, in the prolonged vasospasm one week after SAH, the main role would shift from PKC to tyrosine kinase. Phosphorylation of myosin light chain might not contribute in the mechanism of vasospasm. 
Abtsract \#: $2-8$

A SRC FAMILY PROTEIN TYROSINE KINASE IS INVOVED IN CONTRACTION OF RAT TAIL ARTERIES INDUCED BY A HYPOSMOTIC CONDITIONS

Wijetunge S; Hughes AD.

Clinical Pharmacolgy, NHLI Division, Faculty of Medicine, Imperial College London, W2 1NY. UK

Objective: Hyposmotic conditions cause vasoconstriction due to activation of L-type (CaV1.2) calcium channels [1]. This study examined the role of src family protein tyrosine kinases in this action. Design and Methods: Isometric contraction of rat isolated tail arteries was measured in a myograph containing physiological saline solution with 50mM mannitol, bubbled with 95\% O2 \& 5\% CO2 and maintained at $37^{\circ} \mathrm{C}$. Hyposmotic conditions were achieved by reducing the mannitol concentration. Tyrosine phosphorylation in arterial tissue was examined by SDS PAGE and Western blotting using PY99 anti-phosphotyrosine antibody. Contraction is expressed as \% maximum response to depolarizing potassium solution. Data are means $\pm S E M$ of $\mathrm{n}$ observations. Results: Reducing osmolarity from $300 \mathrm{mOsm} / \mathrm{L}$ to $250 \mathrm{mOsm} / \mathrm{L}$ caused contraction of arteries $(34 \pm 2 \%, \mathrm{n}=37, \mathrm{p}<0.0001)$. The non-selective tyrosine kinase inhibitor, genistein $(10 \mathrm{microM})$, inhibited the contractile response to the hyposmotic stimulus by $92 \pm 4 \%(n=15, p<0.001)$, whereas daidzein $(10$ microM), an inactive analogue did not significantly affect responses. Three structurally unrelated inhibtors of src family tyrosine kinases, herbimycin A (500nM), PP1 (5microM) and SU6656 (10microM) inhibited the hyposmotic response by $42 \pm 8 \% n=5, p=0.005 ; 37 \pm 11 \% n=5, p=0.03$ and $59 \pm 12 \% n=6, p=0.003$ respectively. Exposure to hyposmotic solution for 5 or $10 \mathrm{~min}$ increased tyrosine phosphorylation of several proteins $(n=3)$, including a $60 \mathrm{kDa}$ protein that might be a member of the src family. Conclusions: A member of src family of tyrosine kinases is involved in arterial contraction induced by hyposmotic solutions. Since c-src is found in large amounts in vascular smooth muscle this enzyme is a likely candidate to mediate this effect.

Acknowledgement: This study was supported by the British Heart Foundation 1. Wijetunge S, Hughes AD. Role of voltage-operated calcium channels and tyrosine kinases in the contraction of rat isolated tail artery by hyposmotic conditions Hypertension 2003; 42:647. 


\title{
Abtsract \#: $2-9$ \\ SMOOTHELIN ISOFORMS IN ARTERIAL CONTRACTILE REACTIVITY.
}

\author{
Paquay J, P. Niessen, S. Rensen, J. van Deursen*, G. van Eys, J. De Mey
}

Cardiovascular Research Institute Maastricht, Maastricht, The Netherlands and Mayo Clinic*, Rochester, MN, USA.

Background: In the adult, the expression of the actin-binding protein smoothelin is restricted to smooth muscle. The short isoform (SmtnA) is found in all smooth muscle, while the long isoform (SmtnB) is restricted to vascular smooth muscle being more prominent in small muscular than large elastic arteries. We evaluated the role of smoothelins in arterial contractile reactivity. Material and Methods: Knock out mice deficient in both Smtn isoforms (Smtn-/-), mice lacking the long vascular-selective isoform (SmtnB-/-) and littermate age- and sex-matched wild type mice. Recording of maximal active wall stress (AWS) responses in isolated segments of thoracic aorta (TAO) and femoral arteries (FEM) of 8 week old mice, to $\mathrm{K}+$, the thromboxane analogue U46619 and phenylephrine (PHE). Results: Fetal mortality was not statistically significant in Smtn-/- and SmtnB-/-. During the first 8 weeks of postnatal life, $80 \%$ of Smtn-/died with signs of impaired $\mathrm{Gl}$ and uretheral function while SmtnB-/- grew and survived to the same extent as wild type mice. In TAO, AWS responses to $\mathrm{K}+$ and $\mathrm{U} 46619$ did not differ between strains but AWS responses to PHE were $40 \%$ smaller in Smtn-/- and SmtnB-/- than wild type mice. In FEM, AWS responses to $\mathrm{K}+, \mathrm{U} 46619$, PHE, noradrenaline and angiotensin II were all 40-60\% smaller in Smtn-/- and SmtnB-/compared to wild type. AWS responses were 4 times larger in FEM of wild type mice than in their TAO. In Smtn-/- and SmtnB-/-, AWS responses of FEM were only 1.5-2 times larger in FEM than in TAO.

Conclusion: Smoothelin is not only a marker but also a determinant of the contractile activity of smooth muscle. In the arterial system, SmtnB seems to play a general and non-selective role in the control of the contractile apparatus, while SmtnA might mediate effects of stimuli that activate protein kinase C. 
Abtsract \#: $2-10$

ARTERIAL DYSFUNCTION IN A MOUSE MODEL OF CHRONIC MODERATE VEGF-DEFICIENCY.

De Mey J, G. Fazzi, G. Janssen, J. Paquay, P. Schiffers, R. Hilgers, M. Sanders, E. Storkebaum, P. Carmeliet*.

Cardiovascular Research Institute Maastricht, Universiteit Maastricht, the Netherlands and Center for Transgene Technology and Gene Therapy*, Katholieke Universiteit Leuven, Belgium.

Background: Vascular endothelial growth factor (VEGF) is not only essential for vasculogenesis and angiogenesis but might also have several "housekeeping" functions in the cardiovascular and renal systems of the adult. Furthermore, VEGF is increasingly implicated in neuro-degenerative and neuro-muscular diseases. Suggested mechanisms include, effects of VEGF on neuron survival and on the perfusion of the central nervous system (CNS). We explored the possibility that the peripheral vasculature is involved. Material and Methods: We compared arterial properties in age-matched litter mate wild type mice and in "knock-in" mice that lack the hypoxia-responsive element in the promoter sequence of their VEGF-gene (VEGFd/d mice), several months before onset of amyotrophic lateral sclerosis. Results: Capillary densities were not altered in VEGFd/d mice, but body weight (-50\%), heart weight (-45\%) and kidney weight $(-50 \%)$ were reduced more than brain weight $(-25 \%)$. Therefore, less blood flow can be redistributed from the periphery to the CNS. In line with this suggestion, the diameter and media mass of carotid arteries and middle cerebral arteries were not modified while diameter and media mass of mesenteric resistance and saphenous arteries were reduced. Contractile responses of isolated carotid arteries were not modified, while responses to various vasoconstrictor agonists were reduced (-50 to $-70 \%)$ in mesenteric and saphenous arteries both in terms of wall tension and of wall stress. Contractile responses to sympathetic nerve stimulation were reduced even more than those to exogenous noradrenaline. However, the density of peri-arterial sympathetic nerve fibres and the arterial noradrenaline-content, did not differ between VEGFd/d and wild type mice. Quantitative PCR analyses revealed that the expression of the contractile phenotype marker smoothelin was reduced in saphenous arteries of VEGFd/d mice. Conclusion: Chronic impairment of hypoxia-induced VEGF expression, is accompanied by altered structure and function of the peripheral vasculature. Vascular beds are smaller and are equipped with hyporeactive "dedifferentiated" smooth muscle cells that seem to be disconnected from the sympathetic nervous system. Such mechanism impairs reflex-control of cerebral blood flow and might aggravate neuro-degeneration. 


\title{
Abtsract \#: $2-11$ \\ PRESERVATION OF VASCULAR CONTRACTION DURING AGEING: DUAL EFFECT ON CALCIUM HANDLING AND SENSITIZATION.
}

\author{
Matz RL, Alvarez de Sotomayor M, Schott C, Andriantsitohaina R.
}

Laboratoire de Pharmacologie et Physico-Chimie des Interactions Cellulaires et Moleculaires, CNRS UMR 7034, 74 route du Rhin, B.P. 24, 67401 Illkirch, France.

INTRODUCTION: Vascular ageing is associated with both structural and functional changes that can take place at the level of the endothelium, smooth muscle cells and the extracellular matrix of the blood vessels. Endothelial dysfunction occurs in large as well as in resistance arteries with ageing.

BACKGROUND: The present study was aimed to characterize the effects of ageing on vascular contraction by noradrenaline in rat isolated arteries. METHODS: The existence of vascular bed heterogeneity was investigated in endothelium-denuded conductance (aorta) and resistance (small mesenteric artery, SMA) arteries, with respect to $\mathrm{Ca}(2+)$ handling, $\mathrm{Ca}(2+)$ sensitization or $\mathrm{Ca}(2+)$-independent mechanisms. RESULTS: In both arteries, contractions to noradrenaline were not different between adult and aged rats. In $\mathrm{Ca}(2+)$ - free medium, noradrenaline elicited a transient increase in tension that was reduced by the $\mathrm{Ca}(2+)$ mobilizing agents, ryanodine and thapsigargin, in arteries from adult rats. A loss of the thapsigargin- but not the ryanodine-sensitive component of noradrenaline-induced contraction was observed in the two arteries from aged rats. After depletion of $\mathrm{Ca}(2+)$ stores with noradrenaline, addition of exogenous $\mathrm{CaCl}(2)$ produced a sustained contraction that was decreased to the same extent by the protein kinase C inhibitor, GF 109203X and the tyrosine kinase inhibitor, tyrphostin A-23, in arteries from adult and aged rats. The Rho-associated protein kinase inhibitor, Y-27632, caused identical relaxation of noradrenaline pre-contracted arteries from both age groups. Basal intracellular calcium $([\mathrm{Ca}(2+)](\mathrm{i}))$ was higher in SMA from aged than from adult rats. In addition, the noradrenaline [Ca(2+)](i)-force relationship was significantly shifted to the right in the SMA from aged rats. DISCUSSION: Altogether, these data indicate that responsiveness to noradrenaline is preserved both in conductance and resistance arteries with ageing. The latter results from the association of increased basal $[\mathrm{Ca}(2+)](\mathrm{i})$, changes in $\mathrm{Ca}(2+)$ handling at the level of thapsigargin-sensitive sarcoplasmic reticulum $\mathrm{Ca}(2+)$-ATPases and decreased myofilament sensitivity to $\mathrm{Ca}(2+)$. The setting of this phenomenon within the vascular wall play a role in prevention of age-associated cardiovascular diseases. 


\title{
Abtsract \#: $2-12$ \\ COMPARISON OF AGONIST-INDUCED CALCIUM SENSITISATION PROFILES BETWEEN RESISTANCE ARTERIES HELD UNDER ISOBARIC AND ISOMETRIC CONDITIONS
}

\author{
Shaw L; Austin C; Taggart MJ
}

Smooth Muscle Physiology Group, Cardiovascular Research, University of Manchester, Manchester Royal Infirmary, Oxford Road, Manchester M13 9WL, UK

\begin{abstract}
It has been reported that isobaric-mounted resistance arteries show an increased sensitivity to a number of agonists compared to isometric-mounted arteries (e.g. Falloon et al., 1995). One method whereby excitatory agonists can regulate vascular tone is by stimulating calcium-sensitisation pathways. However, it is uncertain whether these intracellular pathways are activated to different extents in arteries studied under isobaric (i.e. pressurised) or isometric (i.e. wire-mounted) conditions. The aim of this study was to determine, in permeabilised resistance arteries (in which the bathing milieu surrounding the myofilaments can be regulated), the extent of calcium-sensitisation to the alpha-adrenoceptor agonist phenylephrine (PE) or the thromboxane analogue U46619 in pressurised and wire-mounted resistance arteries. Third order mesenteric arteries from Wistar rats $(150-300 \mu \mathrm{m}$ diameter) were mounted either in a pressure myograph (at $60 \mathrm{mmHg}$ ) or in a wire myograph (at $90 \%$ optical length). Following permeabilisation (500 units/ml alpha-toxin plus 10 $\mathrm{MM}$ A23187, 10-15 minutes at room temperature) a reference contraction to pCa4.5 was performed. Following washout agonist-induced calcium-sensitisation was examined by

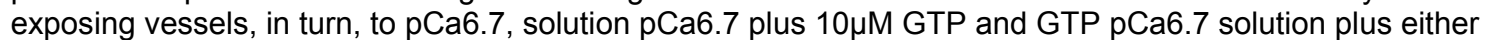
$10 \mu \mathrm{M}$ PE or $10 \mu \mathrm{M} U 46619$. The calcium-sensitising action of U46619 was significantly greater in both pressurised arteries $(45.7+/-8.2 \%$ of $\mathrm{pCa} 4.5$ contraction, $\mathrm{n}=6)$ and wire-mounted vessels $(41.7+/-10.5 \%$ of $\mathrm{pCa} 4.5$ contraction, $\mathrm{n}=7)$ compared to $\mathrm{PE}(16.1+/-3.7 \%$ of $\mathrm{pCa} 4.5$ contraction in pressurised vessels and $10.8+/-2.6 \%$ of pCa4.5 contraction in wire-mounted arteries, $\mathrm{P}<0.05$, Student's Paired t-test). However, there was no difference between isobaric and wire-mounted vessels in the extent of

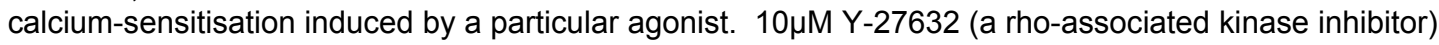
completely inhibited U46619-induced calcium-sensitisation in pressurised $(n=3)$ and isometric $(n=3)$ arteries. In conclusion, the calcium-sensitising actions of PE or U46619 are similar in isobaric or isometric small arteries. Furthermore, thromboxane receptor activation consistently gave greater calcium-sensitisation of tone than alpha-adrenergic stimulation. Supported by the British Heart Foundation Falloon BJ; Stephens N; Tulip JR; Heagerty AM (1995) Am. J. Physiol., 268, H670-H678
\end{abstract}




\title{
Abtsract \#: $2-13$ \\ IN MICE WITH TYPE-2 DIABETES BLOOD PRESSURE IS INCREASED AND MYOGENIC TONE OF ARTERIOLES IS ENHANCED DUE TO COX-2-DEPENDENT TXA2/PGH2 PRODUCTION
}

\author{
Bagi Z, Koller A, Kaley G
}

Department of Physiology, New York Medical College, Valhalla, New York 10595, USA Department of Pathophysiology, Semmelweis University, 1445-Budapest, Hungary

Type 2 diabetes mellitus (T2-DM) markedly increases the incidence of cardiovascular diseases, consequently mortality. T2-DM is frequently associated with elevated blood pressure but the underlying mechanisms are not completely understood. We hypothesized that in T2-DM regulation of peripheral vascular resistance by local mechanisms is altered, which may affect systemic blood pressure. In 12-weeks old male mice with T2-DM (C57BL/KsJ-db-/db-) systolic and mean arterial pressures were significantly elevated compared to those of control littermates (db/db: $146+/-5$ and $106+/-2 \mathrm{mmHg}$, control: $133+/-4$ and $98+/-4 \mathrm{mmHg}$, respectively; $p<0.05)$, measured by a tail cuff method in conscious animals. The calculated total peripheral resistance (obtained from cardiac output - measured by echocardiography- and mean arterial pressure values) was also elevated in $\mathrm{db} / \mathrm{db}$ mice ( $\mathrm{db} / \mathrm{db}: 24+/-6$, control: $15+/-1$ $\mathrm{mmHg} / \mathrm{mL} / \mathrm{min}$ ). In isolated, pressurized gracilis skeletal muscle arterioles (diameter: $\sim 80 \mathrm{~mm}$ ) of $\mathrm{db} / \mathrm{db}$ mice step increases in intraluminal pressure (from $20-120 \mathrm{mmHg}$ ) elicited a greater reduction in diameters compared to control vessels (at $80 \mathrm{mmHg}, \mathrm{db} / \mathrm{db}: 66+/-4 \%$, control: $79+/-3 \%$ of the passive diameters). The passive diameters of arterioles (obtained in $\mathrm{Ca} 2+$-free solution) were not significantly different between the two groups. Presence of SQ 29548, a TXA2/PGH2 receptor antagonist, reduced myogenic tone of arterioles of $\mathrm{db} / \mathrm{db}$ mice back to the control levels (at $80 \mathrm{mmHg}: 80+/-4 \%$ ) but did not affect arterioles of control mice. SC-560, a specific inhibitor of cyclooxygenase-1 (COX-1) did not affect pressure-diameter curves of arterioles. NS-398, a specific inhibitor of COX-2, significantly shifted the pressure-diameter curve upward in $\mathrm{db} / \mathrm{db}$ mice (at $80 \mathrm{mmHg}: 76+/-3 \%$ ), but did not affect that of control vessels. Collectively, these findings suggest that in mice with type 2 diabetes mellitus the pressure-induced myogenic tone of skeletal muscle arterioles is enhanced due to an enhanced COX-2-dependent production of constrictor prostaglandins, TxA2/PGH2, which may contribute to the enhanced peripheral vascular resistance and elevation of systemic blood pressure. (Supported by Hungarian NSRF OTKA, T-034779, and the NHLBI USA HL-46813, HL-43023) 


\title{
Abtsract \#: $2-14$ \\ HYPOXIA-INDUCED DILATION OF HUMAN PRESSURIZED CORONARY RESISTANCE ARTERIES; EFFECTS OF GLIBENCLAMIDE.
}

\author{
Lynch FM; Izzard AS; Austin C; Heagerty AM.
}

Cardiovascular Research Group, Dept. of Medicine, University of Manchester, Manchester Royal Infirmary, Oxford Rd, M13 9WL, UK.

\begin{abstract}
Studies performed in intact guinea pig heart provided evidence that opening of KATP channels are responsible for hypoxia-induced dilation1. We have previously demonstrated a reversal of pinacidil-induced (a KATP channel opener) dilation by glibenclamide (a KATP channel inhibitor) in human coronary resistance arteries. The aims of the current study were to determine the effects of hypoxia on pressurized human coronary resistance arteries with myogenic tone, in vitro. The effects of glibenclamide were also investigated. Human coronary resistance arteries were dissected from atrial appendages obtained from consenting patients undergoing cardiac surgery. Arteries $(n=7)$ were pressurised to $60 \mathrm{mmHg}$ and checked for leaks. Arteries were superfused with physiological saline solution (PSS) at 370C, gassed with $95 \%$ air $/ 5 \%$ CO2. Inner diameter and wall thickness was continually monitored using a video dimension analyzer. Once myogenic tone stabilized arteries underwent a 10 minute hypoxic challenge after which normoxic (95\% air/5\% CO2) conditions were restored. Hypoxia $(<10 \mathrm{mmHg}$ O2) was induced by switching to a $95 \% \mathrm{~N} 2 / 5 \% \mathrm{CO} 2$ gas mixture. In a second group glibenclamide $(5 * 10-6 \mathrm{M})$ was added to the bath at the end of the 10 minute hypoxic challenge. At the end of the experiment, the maximal diameter was assessed by perfusing the vessels in calcium-free PSS. Hypoxia caused a significant $(P<0.05$ paired $t$ test $)$ dilation rat coronary arteries $(n=3)$ with myogenic tone from a mean diameter (+/- SEM) of $108.0+/-2.2$ to $123.2+/$ - 3.8. Return to normoxia was associated with a full reversal of the dilation $(108.6+/-0.4$ micrometers). In a second group $(n=4)$ the diameter change in response to hypoxia from $88.7+/-13.4$ micrometers to $107.1+/-7.8$ micrometers was unaltered by addition of glibenclamide $(107.1+/-5.6$ micrometers). This study demonstrates that hypoxia produces a reversible dilation of human coronary resistance arteries with myogenic tone. The inability of glibenclamide to reverse this response suggests that KATP channels are not involved. Funded by The Wellcome Trust. 1. J. Daut et al. (1990) Science 247:1341 - 1344. 2. F. M. Lynch et al. (2002) Pflugers Archiv. 443: S259.
\end{abstract}




\section{Abtsract \#: $2-15$ \\ CHRONOLOGICAL CHANGES OF ARTERIAL FUNCTION AND HISTOPATHOLOGY IN CANINE "TWO-HEMORRHAGE" VASOSPASM MODEL.}

Koide M; Nishizawa S; Yamaguchi M; Namba H

Department of Neurosurgery, Hamamatsu University School of Medicine, 1-20-1 Handayama, Hamamtsu, 431-3192, Japan

Cerebral vasospasm is a potent and prolonged contraction of cerebral artery following subarachnoid hemorrhage (SAH) and is a potentially fatal consequence of $\mathrm{SAH}$, and influences the prognosis of the patients. Though cerebral vasospasm clinically continues over two weeks, most studies regarding cerebral vasospasm after SAH was carried out for first 7days. The aim of this study is to examine the arterial functions and histopathology of spastic artery for one month. Cerebral vasospasm after SAH was conducted by a canine "two-hemorrhage" model, and angiography was performed to measure arterial diameters once a week. Spastic basilar arteries were excised on days 7, 14, 21, and 28, and following experiments were carried out. Using an isolated spastic artery, arterial functions were analyzed in isometric tension study. Morphological changes were examined by hematoxyline-eosin (HE) and Azan staining. Collagen is a major component of connective tissue, and it's content was measured by quantitative dye-binding method. Immunohistochemical studies of myosin heavy chain (MHC) isoforms (SMemb, SM1 and SM2) were examined to evaluate the phenotype change of smooth muscle cells in the spastic artery. Severe angiographic vasospasm continues until day 14, then resolved at day 21-28 completely after SAH. The vasospastic arteries lost their contractile and relaxant abilities, and the abilities were not improved after recovery from angiographic vasospasm. In the HE staining, the shape of smooth muscle cells were changed from spindle-like shape to shortened and rounded in the spastic arteries. Endothelial detachment and corrugation of internal elastic lamina were also observed at day 7-28. As for Azan staining, staining of connective tissues was obviously increased in the medial layer in the spastic artery, especially at days 14 and 21 . The amount of collagen was once increased at day 14 , although that was returned to the level of the control artery at day 21. Contractile MHCs (SM1 and SM2) were expressed in the control artery and spastic arteries at day 7 and day 28 . Non-contractile MHC (SMemb) increased at days 7-21. These results of immunohistochemial staining indicate that phenotype change of smooth muscle was occurred at day 7-21, especially at days 14 and 21 . Cerebral vasospasm in the canine "two-hemorrhage" model was quite similar in the progression and recovery to that in human. These results indicate that the vasospastic artery increased stiffness of arterial wall despite resolution of angiographic vasospasm, and that phenotype change of smooth muscle contributes in the pathogenesis of vasospasm in later stage. 


\author{
Abtsract \#: $2-16$ \\ REACTIVE HYPERAEMIA OF UPPER LIMBS IS ALTERED WITH LOW BODY NEGATIVE PRESSURE IN \\ HUMANS
}

Charles M; Pichot V; Barthelemy JC; Costes F.

Service EFCR, Hôpital Nord, CHU Saint-Etienne, 42055 Saint-Etienne Cedex 02

Arterial and arteriolar constriction mediated by sympathetic nervous activity has a key role in central blood pressure regulations and reduced peripheral blood flow. However, when metabolic needs of a tissue increase, functional sympatholysis occur and locally prevent sympathetic -induced vasoconstriction. It is due to local vasodilation stimuli constantly involved in the local blood flow regulation and associated to an increased oxygen requirement in the tissue. The purpose of this study was to assess non invasively, the efficiency of muscle sympathetic nerve activation to constrict arterial and arteriolar smooth muscle cells during reactive hyperaemia induced by ischemia. METHODS: To stimulate muscle sympathetic nerve activity specifically and gradually, 3 levels of low body negative pressure (LBNP; $-10,-15,-20 \mathrm{mmHg}$ ) were applied in 6 healthy subjects (30+/-5 years). At rest and during each LBNP level, gradual increased gaps of forearm ischemia $(20,40,60,90,120 \mathrm{sec})$ were performed by inflating a cuff to suprasystolic pressure. Subsequently reactive hyperaemia $(\mathrm{RH})$ were determined immediately after cuff release. Forearm blood flow $(\mathrm{Qb})$ and $\mathrm{RH}$ were measured with a mercury in-silastic strain gauge plethysmography. Blood pressure (Portapres) and heart rate were continuously monitored. Heart rate variability (HRV) analysis has been processed in both time and frequency domains, at rest and for each LBNP level. RESULTS: Central parameters such as blood pressure heart rate and HRV, were not affected by the level of depressurization used. However, LBNP significantly reduced $Q b$ measured on the 2 first minutes of each LBNP stage $(p<0.05)$. RH was reduced by LBNP too. This sympathetic-induced decrease of RH was not significant for short ischemia and more marked on the longest occlusions, particularly on $\mathrm{RH}$ following $120 \mathrm{~s}$ of ischemia, $(31+/-4,25+/-3,23+/-3,21+/-3 \mathrm{ml} /(100 \mathrm{ml}) \cdot \mathrm{min}-1$ at rest and at each LBNP level respectively; $p<0.05)$. $\mathrm{RH}$ was linearly and positively related to the duration of occlusion $(r 2=0.94, p<0,05)$ and the slope of this relationship was decreased with LBNP. DISCUSSION: Sympathetic stimulation partially blocked $\mathrm{RH}$, proportionally to the level of LBNP. However, this effect was surprisingly and essentially observed to the longest occlusion durations contrary to our first hypothesis. In regards to previous studies, it appears that our protocol probably induced additional myogenic arteriolar reflex and additional ischemia related to prior sympathetic -induced constriction due to delay before the first occlusion and between consecutive ischemic procedure. It could have altered the basal arteriolar tone before each occlusion and complicated the interpretation of the data. 


\title{
Abtsrat \#: $2-17$ \\ THE PHYSIOLOGICAL AND PHARMACOLOGICAL CHARACTERISTICS OF MOUSE ISOLATED MIDDLE CEREBRAL ARTERY
}

\author{
Bai N, Moien-Afshari F, Washio H, Min A and Laher I
}

Department of Pharmacology \& Therapeutics Faculty of Medicine, University of British Columbia 2176 Health Sciences Mall Vancouver, British Columbia Canada V6T $1 Z 3$

The routine availability of murine models of various cerebral circulatory disorders requires characterization of the regulation of cerebral artery tone in mouse. Using vasoconstrictors and vasodilators with known efficacy in the cranial circulation of other species, we determined the pharmacological properties of the isolated pressurized mouse middle cerebral artery (MCA). The maximal pressure-induced myogenic constriction in isolated mouse MCA was $20.6 \pm 2.4 \%$. Inhibition of nitric oxide (NO) and endothelin-1 (ET-1) altered the extent of pressure-induced myogenic tone. Isolated mouse MCA failed to either constrict or relax to 5-hydroxytryptamine (5-HT) and histamine; other vasoconstrictors demonstrated the following rank order of efficacy: ET-1 > phenylephrine $>U-46619$. The rank order of endothelium-dependent vasodilator efficacy was bradykinin>acetylcholine> substance $P$. The constriction produced by phenylephrine $(P E)$ required a smaller increase in intracellular $\mathrm{Ca} 2+$ elevation compared to the constriction of a similar magnitude produced by membrane depolarization with potassium chloride $(\mathrm{KCl})$. Pressure-induced myogenic tone $(20 \mathrm{~mm}--80 \mathrm{~mm} \mathrm{Hg})$ in mouse MCA was associated with smooth muscle cell membrane depolarization in mouse MCA $(-52.6 \pm 0.9 \mathrm{mV}$ to $-37.3 \pm 1.7 \mathrm{mV})$. Pressure-induced myogenic tone occurred with a smaller change in membrane potential compared to tone of a similar magnitude produced with $\mathrm{KCl}(-43.4 \pm 2.6 \mathrm{mV}$ vs. $-29.5 \pm 1.0 \mathrm{mV})$. The mouse MCA has a pharmacological profile that is distinct from other species including humans; however, similar to findings in other cerebral arteries, the mouse MCA shows intracellular sensitization to $\mathrm{Ca} 2+$ following receptor activation. 


\section{Abtsract \#: 2-18}

\section{VASOACTIVE PROPERTIES OF LARGE PULMONARY VEINS OF RAT.}

Bronquard C; Maupoil V; Findlay I; Cosnay P; Freslon JL.

UMR CNRS 6542 «Physiologie des Cellules Cardiaques et Vasculaires », Faculté des Sciences et Techniques, Parc de Grandmont, 37200 TOURS, France.

\section{Introduction}

Large pulmonary veins (LPV) present a particular structure with cardiac muscle fibers extending from the left atrium. They can be a source of focal activity which may initiate atrial fibrillation, the most common cardiac arrhythmia. The vasoactive properties of these vessels, and particularly the role of the endothelium, have not been clearly described.

Purposes

In this study we determine : 1) the normalized internal diameter of LPV to determine the optimal static tension before studying vasoactive properties, 2) the responses to various vasoconstrictor and vasorelaxant agents by the construction of Cumulative Concentration-Response Curves (CCRC), 3) the role of the endothelium by using endothelium-dependent and endothelium-independent vasodilators, and $\mathrm{NO}$ and prostaglandin synthesis inhibitors.

Materiel and Methods

Segments of LPV (mean diameter $755+/-20 \mu \mathrm{m}$ ) from male Wistar rats $(400-550 \mathrm{~g}$ ) were mounted in a MulvanyHalpern myograph under normalized tension for measurement of isometric force. Contraction and relaxation to various substances were investigated.

Results

An optimal wall tension corresponding to an in vivo pressure of $10 \mathrm{~mm} \mathrm{Hg}$ in LPV was determined. U46619, a stable analog of thromboxan $\mathrm{A}_{2}$, produced a contraction $\left(\mathrm{pD}_{2}=7.1+/-0.1\right.$, maximal contraction $\left.=0.17 \mathrm{mN} / \mathrm{mm}\right)$, whereas phenylephrine, angiotensin I and II $\left(10^{-9}-10^{-5} \mathrm{M}\right)$ did not contract LPV. In precontracted LPV, isoprenaline induced a relaxation $\left(\mathrm{pD}_{2}=8.2+/-0.1\right.$, maximum $=69 \%$ of the maximal contraction) which was fully inhibited by $10^{-6} \mathrm{M} \mathrm{ICl} 118551$, a beta beceptor antagonist. Salbutamol, a beta $_{2}$-adrenergic agonist, induced a relaxation $\left(\mathrm{pD}_{2}\right.$ $=7.8+/-0.1$, maximum $=56 \%$ of the maximal contraction) and dobutamine, a beta ${ }_{1}$-adrenergic agonist, had no effect below $10^{-7} \mathrm{M}$. Precontracted LPV did not respond to acetylcholine or bradykinin $\left(10^{-9}-10^{-5} \mathrm{M}\right)$ whereas the $\mathrm{NO}$ donor, sodium nitroprusside, induced a relaxation $\left(\mathrm{pD}_{2}=6.8+/-0.2\right.$, maximum $=73 \%$ of the maximal contraction). Neither vasoconstriction nor vasorelaxation were affected by 20 min preincubation with L-NAME (10 $\left.{ }^{4} \mathrm{M}\right)$ and indomethacin $\left(10^{-5} \mathrm{M}\right)$.

\section{Conclusion}

These results show the presence of functional thromboxan $A_{2}$ receptors in LPV whereas alpha- adrenergic and angiotensin II receptors are absent or not functional. Vasorelaxation is related to a beta ${ }_{2}$ - adrenergic mechanism and the endothelium does not seem to have a modulatory role.

Histological studies are in progress to determine the cellular composition of the LPV, in particular, the presence and/or distribution of the endothelium and different receptors. 


\title{
Session 3 : Pressure and flow-induced remodeling of resistance arteries
}

\author{
Ahtsract \#: $3-1$ \\ COMBINED TREATMENT EFFECTS OF LOW DOSES OF PPARALPHA/GAMMA ACTIVATORS ON BLOOD \\ PRESSURE, VASCULAR REMODELING AND INFLAMMATION IN ANG II-INFUSED RATS.
}

De Ciuceis C; Amiri F; Iglarz M; Schiffrin EL.

Clinical Research Institute of Montreal, 110 Pine Avenue West, Montreal, Quebec, Canada H2W 1 R7.

Individually peroxisome proliferator activated receptors (PPAR) alpha and gamma activators prevent hypertension and modulate vascular remodeling and inflammation in angiotensin (Ang) II-induced hypertension. We investigated the effect of combined treatment with low doses of PPAR alpha (fenofibrate) and gamma activators (rosiglitazone) on blood pressure (BP), inflammation and structure and mechanics of resistance arteries after Ang II infusion. Sprague Dawley rats were infused with Ang II (120 $\mathrm{ng} / \mathrm{kg} / \mathrm{min}$ s.c.) and treated with fenofibrate $(30 \mathrm{mg} / \mathrm{kg} / \mathrm{d})$ or rosiglitazone $(3 \mathrm{mg} / \mathrm{kg} / \mathrm{d})$ or a combination of both drugs. During the 7 days, systolic BP was measured by both radio-telemetry and tail-cuff methods. Structure and mechanical properties of third order mesenteric arteries were evaluated on a pressurized myograph, and inflammation markers (NF-kappaB, VCAM-1 and PECAM-1) of mesenteric vessels were studied by Western Blotting. Systolic BP $(\mathrm{mmHg})$ at the end of treatment was increased $(p<0.001)$ by Ang II $(139+/-1)$ compared to the control group (109+/-1). Neither rosiglitazone nor fenofibrate given alone affected SBP (138+/-2, and 138+/-3, respectively), whereas combined treatment with fenofibrate + rosiglitazone prevented Ang II-induced BP increase (122+/-1, $p<0.001 \mathrm{vs.} \mathrm{Ang} \mathrm{II).} \mathrm{Media/lumen} \mathrm{ratio} \mathrm{of}$ resistance arteries was increased in the Ang II-infused rats compared to controls $(8.5+/-0.6 \mathrm{vs} .5 .9+/-0.2$, $p<0.001)$. This increase was prevented only by the combined treatment $(6.8+/-0.9, p<0.01 \mathrm{vs}$. Ang II). No difference was observed in vessel cross-sectional area. Both rosiglitazone alone and the combined treatment prevented increase of VCAM-1 ( $<<0.01$ vs. Ang II) and PECAM-1 expression induced by Ang II (respectively $p<0.01$ and $p<0.001 \mathrm{vs.} \mathrm{Ang} \mathrm{II).} \mathrm{These} \mathrm{findings} \mathrm{suggest} \mathrm{a} \mathrm{synergistic} \mathrm{effect} \mathrm{of} \mathrm{PPAR} \mathrm{alpha}$ and gamma activators on vascular remodeling, likely explained by their pleiotropic anti-inflammatory properties on the vascular wall. Combined treatment with PPAR alpha and gamma activators at subtherapeutic doses, may exert vascular protective effects in hypertension independently of their role on lipid and glucose metabolism. 
Abtsract \#: $3-2$

\title{
A NEW METHOD FOR IN VIVO AND NON-INVASIVE QUANTITATION OF SKELETAL MUSCLE
} PERFUSION IN MICE

\author{
Carlier PG, Bertoldi D, Fromes Y
}

NMR Laboratory, AFM-CEA, Institute of Myology, IFR14, Babinski bldg, Pitié-Salpêtrière University Hospital, 83, Bd de l'Hôpital, 75653 Paris cedex 13, France

Arterial spin labeling (ASL) combined to NMR imaging has demonstrated a potential for mapping perfusion non invasively with good temporal and spatial resolution. In the skeletal muscle, several studies in men and in rats have quantified hyperperfusion during and after exercise, and during post-ischemic reactive hyperemia. Due to the limited perfusion contrast-to-noise ratio of these sequences, it was uncertain whether ASL could detect perfusion at rest in skeletal muscle. Also, there was little data available on skeletal muscle perfusion in mice, and this despite the pivotal role of this species in modern biomedical sciences. For these reasons, we investigated whether skeletal muscle blood flow could be determined with ASL in the mouse leg, under basal, ischemic and hyperemic conditions. All NMR imaging experiments were performed on a $4 \mathrm{~T}$ magnet. Perfusion was measured with SATIR, an ASL variant, using a fast spin echo imaging sequence, with a $7.85 \times 2.00 \mathrm{~cm}$ FOV and a $128 \times 32$ matrix size. Imaging slice thickness was $2 \mathrm{~mm}$. Echo spacing was $2.4 \mathrm{msec}$ and repetition time was $10 \mathrm{sec}$ to allow full relaxation of the inverted arterial spins. Experiments were performed on 5 female mice weighing $40+/-6 \mathrm{~g}$, anesthetized with isoflurane inhalation and placed on a water heating pad. To perform ischemia of the leg, two threads, as near as possible to the hip formed two loops and interrupted blood flow when pulled. The calf of the mouse was placed facing the surface coil centered in the volume coil. The protocol consisted in measuring perfusion during a basal protocol of $7 \mathrm{~min}$, followed by an ischemic period of 7 min, and finally monitoring the post-ischemic hyperemic response during $15 \mathrm{~min}$. Perfusion was calculated from signal intensities measured on a region-of-interest in the calf muscles which excluded lipids, bone and vessels. Mean basal perfusion was measured at 18.4+/-3.6 $\mathrm{ml} / \mathrm{min} / 100 \mathrm{~g}$ and mean perfusion under ischemic condition was $2.1+/-3.5 \mathrm{ml} / \mathrm{min} / 100 \mathrm{~g}$ ( $\mathrm{p}=0.0003$ vs baseline values). When the tension of the threads was released, a characteristic peak of hyperemia (ranging from 49 to 115 $\mathrm{ml} / \mathrm{min} / 100 \mathrm{~g}$ ) was detected, after which perfusion returned to basal values. These results suggest that not only hyperperfusion states but also relatively low levels of perfusion can be assessed in the skeletal muscle of mice with ASL. This opens interesting new prospects for the in vivo and non-invasive investigation of resistance vessel control in murine models. 
Abtsract \#: $3-3$

TRANSGLUTAMINASE IS CRUCIAL IN INWARD REMODELING OF SMALL CORONARY ARTERIES

Bakker ENTP; Perree J; Ganga A; Sorop O, Spaan JAE and VanBavel E.

Department of Medical Physics, Cardiovascular Research Institute Amsterdam, Academic Medical Center, Amsterdam, the Netherlands

Vascular remodeling results in a change in arterial diameter under fully dilated conditions. This may occur without apparent changes in the amount of cellular and extracellular wall constituents. We hypothesized that such eutrophic remodeling is determined by the cross-linking of existing extracellular matrix proteins by enzymes from the transglutaminase family. Small coronary arteries (200 microns) were isolated from young female farm pigs (20-25 kg). The expression of tissue-type transglutaminase (tTG) was confirmed by RT-PCR and sequence analysis. Arteries were maintained in organoid culture for 3 days at $60 \mathrm{mmHg}$, during which strong vasoconstriction developed. Comparison of the maximal diameter on day 0 to day 3 showed inward remodeling, of $-9+/-3 \%$ at $60 \mathrm{mmHg}$ (mean $+/-\mathrm{SEM}, \mathrm{n}=7$ ). Immunostaining showed the appearance of a specific cross-link in the medial layer of remodeled arteries. Upregulation of tTG with retinoic acid (10-7 M) resulted in increased vasoconstriction and doubled inward remodeling: -18 $+/-4 \%(n=6)$. Inhibition of tTG during culture with cadaverine induced vasodilation and reversed remodeling to a substantial increase in maximal diameter from day 0 to day $3:+26+/-5 \%(n=5)$. Direct exposure of arteries to transglutaminase induced inward remodeling already at 24 hours: $-10+/-3 \%(n=9)$ vs. $+2+/-1 \%$ in control arteries $(n=7)$. The effect of exogenous transglutaminase was completely inhibited by simultaneous addition of the nitric oxide donor nitroprusside $(0.1 \mathrm{mM}):+4+/-2 \%(\mathrm{n}=6)$. Further study of the interaction between smooth muscle cells and extracellular matrix was done using a collagen gel contraction assay. Cultured coronary smooth muscle cells (10E5) compact collagen, to $64+/-3 \%$ of the original gel diameter after $24 \mathrm{~h}$. Exogenous transglutaminase significantly enhanced gel compaction to only $23+/-3 \%$ of the original diameter. Inhibition of tTG reduced compaction, to $89+/-2 \%$ of the original gel diameter. In conclusion, these data reveal a novel mechanism of vascular remodeling, based on tissue-type transglutaminase. Supported by the Netherlands Heart Foundation, NHF grants 2001D038 and 98.180 . 


\title{
Abtsract \#: $3-4$ \\ CHANGES IN BRADYKININ AND ANGIOTENSIN II TYPE 2 RECEPTOR-DEPENDENT TONE AFTER CHRONIC CHANGES IN BLOOD FLOW IN MESENTERIC RESISTANCE ARTERIES.
}

\author{
Cousin M, Baron C, Dumond O, Custaud MA, Loufrani L, Henrion D.
}

CNRS UMR 6188, University of Angers, France.

Chronic changes in blood flow in resistance arteries induce a vascular remodeling. We have previously shown that local renin-angiotensin and Kallikrein-kinin systems are involved in flow-dependent dilation through the activation of angiotensin II type 2 (AT2R) and bradykinin type 2 (B2R) receptors. Thus, it is likely that these 2 systems may play a role in flow-induced remodeling. The aim of the present study was thus to explore the role of angiotensin receptor type ATR2 and B2R in resistance arteries submitted to chronic increase or decrease in blood flow. We submitted rat mesenteric resistance arteries $(250 \mu \mathrm{m}$ diameter) to chronic changes in flow by alternatively ligating arteries. Arteries were thus submitted to high (HF), low (LF) or normal flow (NF). After 2 weeks, arteries were studied in vitro in a myograph for resistance arteries.

Two weeks after ligation, arterial diameter was increased in HF arteries and bradykinin-induced dilation decreased (no change in AT2R- and acetylcholine-dependent dilation). In HF arteries, bradykinin-induced dilation was restored to normal after acute AT2R blockade, suggesting that AT2R exerted a vasoconstrictor effect. In LF arteries, arterial diameter was decreased, bradykinin-dependent dilation unaffected, but AT2R-dependent dilation increased (no change in B2R- and acetylcholine-dependent dilation). Acetylcholine-induced dilation was decreased in LF arteries and unaffected in HF arteries. Thus, chronic changes in flow increased AT2R-dependent tone in mesenteric arteries with AT2R-dependent contraction in HF arteries and AT2-dependent dilation in LF arteries. These changes in the response obtained after AT2R stimulation might reflect a opposite effect of AT2R in the remodeling of LF and HF arteries. 


\title{
Abtsrat \#: $3-5$ \\ RESISTANCE ARTERIES OF ANG II-INFUSED OSTEOPETROTIC MICE SHOW REDUCED IMPAIRMENT OF ENDOTHELIAL FUNCTION, OXIDATIVE STRESS AND VASCULAR REMODELING.
}

\author{
De Ciuceis C; Amiri F; Endemann DH; Touyz RM; Schiffrin EL.
}

Clinical Research Institute of Montreal, 110 Pine Avenue West, Montreal, Quebec, Canada H2W 1 R7.

\begin{abstract}
Vascular damage induced by angiotensin II (Ang II) may be partially mediated by vascular inflammation and reactive oxygen species generation. Homozygous osteopetrotic (op/op) mice lacking macrophage colony-stimulating factor (mCSF) showed an inhibition of atherogenesis in models of atherosclerosis. However, no data are presently available about the role of mCSF and its effects on hypertension. We therefore investigated the effects of Ang II on structure and function, and NADPH oxidase activity in resistance mesenteric arteries from op/op mice. Adult op/op, heterozygous (op/+), and wild type $(+/+)$ mice were infused with Ang II (1000 ng/kg/min, sc) or vehicle for 14 days. Systolic blood pressure (SBP) was measured by the tail-cuff method. Endothelium-dependent relaxation to acetylcholine (Ach), with or without L-NAME, and vascular structure, were assessed in mesenteric resistance arteries mounted on a pressurized myograph. NADPH oxidase activity was evaluated by lucigenin chemiluminescence. Ang II similarly elevated SBP $(\mathrm{mmHg})$ in $+/+$ and op/ $(163+/-9$ and $160+/-10, P<0.01$ vs. controls), and in homozygous mice (143+/-7, $\mathrm{P}<0.05 \mathrm{vs}$. control). Mesenteric arteries from Ang II-treated op $+/+$ and op/+ mice exhibited reduced Ach-mediated-relaxation (maximal relaxation respectively $64 \%$ and $67 \%$ vs. $84 \%$ and $93 \%$ in controls, $\mathrm{P}<0.05$ ), which was unaffected by L-NAME. Op/op mice infused with Ang II showed significantly less endothelial dysfunction as measured by Ach-induced relaxation $(87 \%$ vs. $96 \%$ in controls), which could be further impaired by pre-incubation with L-NAME. Arteries from Ang II-infused +/+ and $\mathrm{op} /+$ mice showed increased media:lumen ratio $(P<0.01)$ and media thickness $(P<0.01)$, neither of which were different in op/op mice compared with untreated counterparts. Media cross sectional area was increased $(\mathrm{P}<0.05)$ by Ang II only in $+/+$ mice. NADPH oxidase activity was increased by Ang II infusion in $\mathrm{op}+/+(\mathrm{P}<0.05)$ and unchanged in $\mathrm{op} /+$ and op/op mice compared to controls. These findings indicate that mCSF-deficient mice were more resistant to endothelial dysfunction, vascular remodeling and oxidative stress induced by Ang II, suggesting a critical role of proinflammatory mediators in vascular injury associated with Ang II-induced hypertension.
\end{abstract}




\title{
Abtsract \#: 3-6 \\ FLOW-INDUCED VASCULAR REMODELING IS IMPAIRED IN THE MESENTERIC ARTERY OF SPONTANEOUSLY HYPERTENSIVE RAT
}

\author{
Gao Yu-Jing; Yang Lu-Fang; Stead Shelley; Lee Robert M.K.W.
}

Smooth Muscle Research Program and Department of Anaesthesia, McMaster University, 1200 Main Street West, Hamilton, Ontario, Canada L8N $3 Z 5$

The effect of increased blood flow on vascular remodeling was studied in the mesenteric arteries of 10-15-week-old spontaneously hypertensive rats (SHR) and age-matched normotensive Wistar-Kyoto rats (WKY). Increased flow was induced by selective ligation of mesenteric arteries. Nearby arteries with normal blood flow were used as controls. 7-10 days after the ligation procedure, mesenteric arteries were fixed in situ at maximal relaxation by perfusion fixation. Morphometric measurement of vascular dimension was carried out using confocal microscopy. Apoptotic cells were detected by the terminal uridine nick-end labelling method. Cell growth was quantified using proliferating cell nuclear antigen (PCNA) in sections of paraffin-embedded vessels. In SHR, elevated blood flow increased the vessel wall dimension and the number of smooth muscle cell (SMC) layers, increased wall to lumen ratio and the number of PCNA-positive SMC, but no change in the lumen size or the number of apoptotic SMC. In WKY increased blood flow resulted in an increase in lumen diameter, a reduction of apoptotic SMC, but no change in wall to lumen ratio, the number of SMC layers, or the number of PCNA-positive SMC. These results showed that mesenteric arteries from hypertensive and normotensive rats respond to an increase in blood flow differently: a lumen enlargement with reduced SMC apoptosis in WKY, but an increased wall to lumen ratio with enhanced SMC growth in SHR. It remains to be determined if flow alteration is one of the initiating factors in the development of vascular remodeling in hypertension. 


\title{
Abtsract \#: $3-7$ \\ TIME COURSE EXPRESSION OF STRUCTURAL AND REGULATORY PROTEINS OF FOCAL ADHESIONS IN RESISTANCE ARTERIES DURING EUTROPHIC REMODELING
}

\author{
Girardot D, deBlois $D^{*}$ and Moreau $P$
}

Faculté de pharmacie, Université de Montréal, *Centre de recherche”, Hôtel-Dieu, Montréal, Canada.

Introduction: L-NAME-induced hypertension leads to hypertrophic remodeling in large arteries and to eutrophic remodeling (decreased internal diameter without changing cross sectional area) in small arteries. Background/purposes: during eutrophic remodeling, smooth muscle cells are expected to reorganize their cytoskeleton and to alter the signaling pathways that regulate contractile protein activation and function. Focal adhesions, which offer a structural link between the actin cytoskeleton and the extracellular matrix elements, are composed of numerous structural and regulatory proteins such as vinculin and $\mathrm{p} 140 \mathrm{mDia}$. Our objective was to measure the time course expression of proteins implicated in the structural (vinculin) and regulatory formation (p140mDia) of focal adhesions in a model of eutrophic remodeling of resistance arteries and compare the results with a model of hypertrophic remodeling. Material/methods: male Wistar rats were treated with L-NAME at a dose of $50 \mathrm{mg} / \mathrm{kg} /$ day during 1, 3, 6 and 24 hours, and 3, 7 and 14 days. Another group of rats received angiotensin II by osmotic pumps at a dose of $200 \mathrm{ng} / \mathrm{kg} /$ day during the same time course. A third group of rats served as control. Mesenteric arteries (excluding the superior mesenteric artery) were harvested and used for measurement of protein expression by western immunoblotting. Results: in mesenteric arteries, vinculin expression decreased during 1 to 6 hours of L-NAME administration (1h: $18,7+/-4,3 \%, 3 \mathrm{~h}: 34,0+/-8,6 \%$, $6 \mathrm{~h}: 35,8+/-6,9 \%$; $\mathrm{p}<0,05)$. In angiotensin II-treated rats, vinculin expression was also decreased from $3 \mathrm{~h}$ to 3 days ( $3 \mathrm{~h}$ : 38,6+/-10,4\%, 6h: 45+/-8,8 \%, 24h: 34,7+/-12,7\%, 3 days: 36,6+/-7,6\%; p<0.05). In contrast, p140mDia expression was reduced only at day 14 in L-NAME treated $(34.7+/-3,5 \% ; p<0,05)$., and was unchanged by Ang II administration. Discussion: in resistance arteries, vinculin is reduced early during both eutrophic and hypertrophic remodeling, suggesting that focal adhesions may be reduced during hypertension-induced remodeling, irrespective of its type. In contrast, the regulatory protein (p140mDia) seemed only involved in eutrophic remodeling, albeit at a later stage of the remodeling process. 
Abtsract \#: $3-8$

IS ENDOTHELIN A TROPHIC OR MITOGENIC PEPTIDE IN SMALL ARTERIES IN VIVO?

\author{
Dao HH; Beaucage P; Moreau P
}

Faculty of Pharmacy, Université de Montréal, Montréal, Québec, Canada H3C 3J7

\begin{abstract}
We have previously reported that chronic intraperitoneal administration of endothelin $(5 \mathrm{pmol} / \mathrm{kg} / \mathrm{min}$ for 4 weeks) induces mesenteric artery hyperplasia. Acutely (24 hours), both protein and DNA synthesis were enhanced in mesenteric arteries, and this was associated with a reduced binding of p27Kip1 to CKD2 and an enhanced activity of the cyclin-dependent kinase. Although the dose used produced local ET concentrations within the pathophysiological range $(3.71+/-0.76$ vs $1.48+/-0.13 \mathrm{pg} / \mathrm{mg}$ in control rats), our results could not explain that in other conditions ET leads to vascular hypertrophy, such as in the chronic angiotensin II and norepinephrine models. Our objective was to test the hypothesis that lower concentrations of ET would produce only vascular protein synthesis. Rats were treated for 24 hours with one of several doses of ET $(0.03,0.1,0.3,1,3$ and $10 \mathrm{pmol} / \mathrm{kg} / \mathrm{min})$ and protein synthesis was measured in small mesenteric arteries collected from those rats. DNA synthesis was also measured at $1 \mathrm{pmol} / \mathrm{kg} / \mathrm{min}$ ET. We observed a dose-dependent increase of protein synthesis with a half-maximal effect at 0.35 and a maximal effect at $1 \mathrm{pmol} / \mathrm{kg} / \mathrm{min}$. Blood pressure measured in awake rats was not increased by these local administrations. In contrast to what was observed at $5 \mathrm{pmol} / \mathrm{kg} / \mathrm{min}$, DNA synthesis was not elevated in rats treated with $1 \mathrm{pmol} / \mathrm{kg} / \mathrm{min}$. Moreover, CKD2 activity was also normal. Our results suggest that the effect of ET on protein and DNA synthesis are dissociated and are dose related, suggesting different signaling events (currently investigated). Thus, ET can produce hypertrophic or hyperplasic responses in vivo with very small changes in local concentrations, suggesting that both responses could occur in small arteries depending on the severity of the disease and the local ET concentration.
\end{abstract}




\section{Abtsract \#: $3-9$ \\ STRUCTURE AND FUNCTION OF CORONARY RESISTANCE ARTERIES CULTURED WITH FLOW}

Pistea A, Bakker EN, Spaan JAE, VanBavel E

Department of Medical Physics, Academic Medical Center, University of Amsterdam, 1100 DE Amsterdam, The Netherlands

Introduction: The caliber of blood vessels is known to depend on the flow they carry. Shear stress is believed to be a major determinant in this adaptation. However, the mechanisms of flow-induced remodeling are poorly understood, especially in the coronary circulation, where flow may change with stenosis. Our previous experiments using organoid culture of small vessels have shown inward remodeling in vessels kept at constant pressure, with no flow. We hypothesized that adding flow to this in vitro setting causes adaptation of active diameter by tone and remodeling in order to yield a desired shear stress, and inhibits inward remodeling. Methods: We developed an organoid culture setup able to drive a flow through cultured cannulated arterioles at constant luminal pressure, using a pressure gradient between the pipettes. Subepicardial porcine coronary arterioles, having a diameter at full dilation and $60 \mathrm{mmHg}$ (D0) of 159+/-3 \&\#956;m (mean+/-SEM), were isolated and mounted in a pressure myograph at $60 \mathrm{mmHg}$. Vessels (with or without L-NNA; for each condition: study group: with flow; control group: no flow; paired experiments, $n=5$ ) were studied over a 3 day culture period. Results: Bradykinin 10-7 M caused full dilation in all groups, with or without L-NNA, both at the start and end of culture period. Myogenic tone was not significantly different in flow vessels (without L-NNA: active diameter in the first, second and third day was respectively $38.1+/-11.0 \%, 45.8+/-11.1 \%, 49.6+/-7.2 \%$ of DO, vs. $41.6+/-8.4 \%, 44.2+/-15.2 \%$, and $45.7+/-9.4 \%$ in controls; with L-NNA: active diameter in the first, second and third day respectively was $42.7+/-7.5 \%, 60.5+/-9.6 \%, 55.1+/-6.0 \%$ of D0, vs. $63.8+/-10.2 \%, 54.8+/-8.0 \%$, and $43.9+/-4.6 \%$ in controls). In the last day of culture the shear stress in the active vessels without L-NNA became remarkably constant (8.3+/-1.9 dynes/cm2); with L-NNA, shear stress was still slightly increasing (average: 15.0+/-1.0 dynes/cm2). Without L-NNA, passive pressure-diameter curves at the end of culture revealed inward remodeling in the control group $(88.7+/-2.6 \%$ of $D 0)$, no remodeling in the flow group $(100.9+/-1.7 \%$ of D0); with L-NNA, the flow group had inward remodeling (91.1+/-4.0\% of D0). Discussion: Pressurized coronary resistance arteries can be maintained in culture for several days with flow. Vessels were able to readjust their active diameter aiming to a specific value of shear stress, greater if NO synthesis was inhibited. Inward remodeling occurred in the 'no flow' control vessels, was inhibited by flow and unmasked in flow experiments with NOS inhibition. 


\section{Abtsrat \#: $3-10$ \\ CONTRIBUTION TO THE MODELLING AND SIMULATING OF THE CARDIOVASCULAR SYSTEM: FIRST STUDY}

L'Huillier JP; Humeau A

Ecole Nationale Supérieure d'Arts et Métiers (E.N.S.A.M.), Laboratoire Procédés - Matériaux Instrumentation (L.P.M.I.), 2 boulevard du Ronceray, BP 3525, 49035 Angers cedex, FRANCE Groupe ISAIP-ESAIP, 18 rue du 8 mai 1945, BP 80022, 49180 Saint Barthél

Introduction The range of cardiovascular simulations extends from electrical analog and numerical models to a variety of hydromechanical models in order to describe some characteristics and phenomena such as control systems, impedance spectra and fluid mechanics of the heart and blood vessels. This work presents a numerical model of the circulatory system based on the computation of three sets of equations: equations of motion, continuity equations, and ventricle equations of state. Background and purposes The aim of this study is to simulate the pressure in the right and left ventricles and in the aorta, the left ventricle ejection, and the pressure-volume relationship for the active and relaxed states of the left ventricle. Materials and methods The basic system modelling the biomechanical circulation is subdivided into 10 sections. For each section the three above mentioned basic equations are written. Each equation refers to a pressure gradient, input and output flow rates, initial and residual volumes, blood viscosity and inertial properties. The heart pump is defined as time changes in elasticity properties and incorporates the aspect of force-velocity relationship of the myocardium. This constitutes 24 unknowns and 24 associated equations. This set of equations is solved by means of the Runge-Kutta procedure that is well suited for numerical computing. Results and Discussion Among the simulations a study is carried out on the effects of different parameter values on the above mentioned time-dependent events. The results obtained are close to those observed in clinical routines. For example, some curves show the active and passive isometric pressure-volume loop that defines the pressure-volume combination traversed by the ventricle in one cycle. The simulations of the variables issued from other sections could lead to a deeper understanding of the role played by the arteries status on the physiological data. The work can also be used to explain the influence of the diseases on the whole circulation. 


\title{
Abtsrat \#: $3-11$ \\ ROLES OF ANGIOTENSIN II AND BRADYKININ IN FLOW-RELATED ARTERIAL REMODELING IN MICE
}

\author{
Hilgers RHP, Schiffers PMH, Bergaya S, Boulanger C, De Mey JGR
}

Cardiovascular Research Institute Maastricht (CARIM), Universiteit Maastricht, UNS 50, 6200 MD Maastricht, the Netherlands. Institut National de la Santé et de la Recherche Médicale (INSERM), Unit 541, Hôpital Lariboisière, 41 Blvd de la Chapelle, F-75475

Objective. In the arterial wall, membrane-bound angiotensin-converting enzyme (t-ACE) generates angiotensin II and degrades bradykinin, which can be produced by tissue kallikrein (TK). Bradykinin can be released by shear stress in both large conduit arteries and small resistance-sized arteries, and angiotensin II has been shown to induce vascular smooth muscle cell growth. The aim of this study was to evaluate the role of bradykinin and angiotensin II during arterial remodeling in response to chronic changes in blood flow in vivo. Methods and Results. We applied unilateral carotid artery ligation and studied uterine arteries before, during and after pregnancy in t-ACE-/- mice, TK-/- mice, and wild-type mice. In ligated carotid arteries of TK-/- mice the inward remodeling in response to a 4-week blood flow cessation was more substantial than in wild-type mice. Ligated carotid arteries of t-ACE-/- mice, showed a reduced luminal shrinkage compared to ligated arteries of wild-type mice. The medial hypertrophy in ligated arteries was significantly smaller in t-ACE-/- mice compared to TK-/- and their wild-type counterparts. Deficiency of either tissue kallikrein or tissue ACE did not alter the outward hypertrophic remodeling of the uterine artery that occurs during pregnancy, and the subsequent inward hypotrophic remodeling that was complete 7-days postpartum. Conclusions. Bradykinin protects against excessive inward remodeling in response to chronic cessation of blood flow. Angiotensin II induces medial hyperplasia in these flow-limited arteries. Both tissue kallikrein and tissue ACE are not essential for the pregnancy-related uterine artery structural changes, suggesting that adaptive structural arterial changes differ between large elastic arteries and small pre-existing collateral arteries. This opens new perspectives for more site-directed interventions. 
Abtsrat \#: $3-12$

UTERINE ARTERY DILATATION AND REMODELING DURING POSTPONED MURINE PREGNANCY

Hilgers RHP, Schiffers PMH, Fazzi GE, De Mey JGR

Department of Pharmacology \& Toxicology, Cardiovascular Research Institute Maastricht (CARIM), Universiteit Maastricht, UNS 50, 6200 MD Maastricht, the Netherlands.

Objective. Aging was reported to reduce endothelium-dependent vasodilatation and outward arterial remodeling. We hypothesize that this (1) also applies to small muscular arteries in the uterine vascular bed and (2) adversely affects reproductive performance. Methods and Results. We monitored uterine artery vasomotor responses, structural changes and fetal number before and at the 18th day of first pregnancy in approximately 17 (young) and 58 (middle age) weeks old mice. Uterine arteries were larger, but norepinephrine-induced constriction and dilatation in response to flow, acetylcholine, and Na-nitroprusside were comparable in middle age compared to young mice. Nw-nitro-L-arginine (1) reduced dilator responses to acetylcholine in both young and middle age mice, but (2) reduced flow-induced dilatation in young, but not middle age mice. Pregnancy had minimal effects on uterine arterial vasomotor responses in young mice, but enhanced myogenic tone in middle age mice. At term pregnancy, the increase in internal diameter, and in media cross-sectional area and the number of viable fetuses were significantly smaller in middle age than young mice. Conclusion. Aging modifies shear stress-induced vasodilatation and blunts pregnancy-related remodeling of the uterine artery. This might contribute to fetal growth retardation and mortality during postponed pregnancy. 


\title{
Abtsract \#: $3-13$ \\ LOCATION AND FUNCTIONAL ROLE OF VPAC1, VPAC2 AND NPR-C RECEPTORS IN VIP-MEDIATED VASODILATION OF PORCINE CEREBRAL ARTERIES.
}

\author{
Grant S; Lutz EM\#; Robberecht $\mathrm{P}^{*}$; McPhaden $\mathrm{AR}^{\wedge}$ \& Wadsworth RM.
}

Department of Physiology \& Pharmacology and \#Department of Bioscience \& Biotechnology, University of Strathclyde, Glasgow, Scotland, UK, G4 ONR. *Université Libre de Bruxelles, School of Medicine, Belgium. ^Department of Pathology, Glasgow Royal Infirma

\begin{abstract}
Vasoactive Intestinal Polypeptide (VIP) is a major cerebrovascular neurotransmitter peptide present in cerebral intramural nerves. VIP binds to VPAC1, VPAC2 and NPR-C receptors to elicit relaxation of vascular smooth muscle cells. The receptors mediating VIP-induced cerebral vasodilation are unknown and were investigated. To identify receptor mRNA, standard RT-PCR was performed. Rings of porcine basilar arteries, pre-contracted with U-46619, were studied for their vasodilator response to selective VPAC1, VPAC2 and NPR-C receptor agonists. Transmural nerve stimulation (TNS) was delivered to induce reproducible, transient vasodilation. The role of VIP receptors in the modulation of the relaxant response to TNS was investigated by incubation of VPAC1, VPAC2 and NPR-C receptor antagonists. Location of the VIP receptors was determined by immunocytochemistry. The mRNA of VPAC1, VPAC2 and NPR-C receptors was detected by RT-PCR and confirmed by comparison to positive controls. Pig basilar artery rings suspended in a wire myograph relaxed to VIP, the selective VPAC1 agonist, [Lys15Arg16Leu27]VIP(1-7)/GRF(8-27), VPAC2 agonist (Ro-25-1553) and the NPR-C agonist (cANP(4-23)) in a concentration dependent manner. $100 \mathrm{mM}$ L-NAME significantly reduced the relaxation caused by VIP, suggesting NO is involved in the vasodilation. Additional evidence of receptor involvement was obtained with specific antagonists. 1mM [AcHis1DPhe2K15R16L27] VIP(1-7)GRF(8-27), 0.1mM PG-99465 and $10 \mathrm{mM} A N P(1-11)$ all attenuated the relaxation caused by VIP, demonstrating the involvement of VPAC1, VPAC2 and NPR-C receptors respectively. Neurogenic relaxation elicited by TNS ( 8 and $20 \mathrm{~Hz})$ was abolished by $100 \mathrm{mM}$ L-NAME and $0.3 \mathrm{mM}$ TTX. The TNS-induced vasodilation at $20 \mathrm{~Hz}$ was attenuated by pre-incubation with PG-99-465 (VPAC2 antagonist). The VPAC2 receptor is distributed in the outer layers of the media, suggesting that endogenous VIP is released from intramural nerves to induce vasodilation. The VPAC1 receptor is expressed exclusively in the endothelium. Vasodilation induced by the VPAC1 agonist was significantly reduced by L-NAME and abolished by removal of the endothelium. The NPR-C receptor is widely distributed throughout the artery wall, including nerves. The relaxation induced by the NPR-C agonist was significantly attenuated by NOS inhibition. In conclusion, we have presented novel molecular and functional data providing evidence for the involvement of VPAC1, VPAC2 and NPR-C receptors in VIP-induced vasodilation of the porcine basilar artery. The VPAC1 receptor is located only in the endothelium and induces NO-dependent vasodilation. The VPAC2 receptor, present in the outer layers of the media, appears to be involved in vasodilation due to VIP release from intramural nerves. NPR-C receptors are present throughout the artery, including nerves, and are NO-dependent. SG is the recipient of a British Heart Foundation PhD studentship (FS/2000077).
\end{abstract}




\title{
Abtsract \#: 3-14 \\ ACTIVATION OF RAT CREMASTER ARTERIES BY ENDOTHELIN-1 IN A ONE-DAY CULTURE INDUCES INWARD REMODELLING: A POSSIBLE ROLE FOR TISSUE TRANSGLUTAMINASE
}

\author{
Bramsen LH; Buus CL; Sørensen KS; Bakker ENTP*; vanBavel E*; Mulvany MJ
}

Department of Pharmacology, University of Aarhus, 8000 Aarhus C, Denmark *Department of Medical Physics, Academic Medical Center and Cardiovascular Research Institute Amsterdam, (CRIA), Amsterdam, The Netherlands.

Inward eutrophic remodelling of resistance arteries is associated with essential hypertension. We have previously shown that arteries contracted with either endothelin-1 (ET-1) or potassium and pressurized during a three-day period in organ culture, showed inward remodelling. We hypothesized that the contraction primes the artery for structural changes involving cross-linking of pre-existing structures of the extracellular matrix, and that a candidate enzyme for the cross-linking activity is the tissue transglutaminase (tTG). In the present experiments, the culture period was reduced to 1 day. In the first protocol, resistance arteries were cannulated, as previously described. Initially a passive pressure-diameter relation was determined in the presence of $0.1 \mathrm{mM}$ papaverine. After setting pressure to $75 \mathrm{mmHg}$ and washout of papaverine, arteries developed spontaneous tone. Test arteries were then incubated with 10 $\mathrm{nM}$ ET-1, and a strong persistent vasoconstriction was developed. The passive diameter-pressure relation was again determined after washout and in the presence of papaverine. The relaxed diameter at 60 $\mathrm{mmHg}$ was reduced from $165+/-4$ um (fresh) to $154+/-2$ um (day $1, \mathrm{n}=6$ ), compared to control arteries incubated without ET-1: $168+/-2$ um (fresh) and 169+/-2 um (day 1, n=6), indicating inward remodelling $(P<0.05)$. In a second protocol, transglutaminase $(0.1 \mathrm{U} / \mathrm{ml})$ was added to arteries kept at a narrow diameter by reducing pressure to $4 \mathrm{mmHg}$. This protocol also caused inward remodelling of $24 \mathrm{um}(n=6)$; this remodelling could be inhibited by the competitive inhibitor, monodansylcadaverine $(0.1 \mathrm{mM}, \mathrm{n}=6$, $\mathrm{P}<0.05)$. In a third protocol, we found that remodelling caused by contraction with ET-1 can be inhibited by blocking endogenous tTG with the active site inhibitor, cystamine $(n=6, P<0.05$ Immunostained cross-sections of the rat cremaster muscle showed tTG positive staining in the arteries and veins as well in media and adventitia. In the striated muscle the endomysium was ITG positive, while the myofilaments were negative. In conclusion, we have detected inward remodelling after one day in culture. Our results support the possible involvement of transglutaminase in the remodelling process. 
Abtsract \#:3-15

ACTIVATION OF RAT CREMASTER ARTERIES BY ENDOTHELIN-1 IN A ONE-DAY CULTURE INDUCES ALTERATIONS OF VASCULAR REMODELING IN SMALL MESENTERIC ARTERIES FROM AGED RATS

\author{
${ }^{1}$ Briones $\mathrm{AM},{ }^{2}$ Giraldo $\mathrm{J}$ and ${ }^{1}$ Vila E
}

${ }^{1}$ Dpt. Farmacologia, Terapèutica i Toxicologia and ${ }^{2}$ Grup de Modelització Estructural i Funcional de Sistemes Biològics. Facultat de Medicina. Universitat Autònoma de Barcelona. 08193 Barcelona, Spain.

Introduction: The process of ageing is associated with marked changes in the cardiovascular system that can lead to the development of cardiovascular diseases.

Background and purposes: Mesenteric arteries from aged rats are associated with hypertrophy of the vascular wall (Moreau et al., 1998 Cardiovasc Res 37: 247-253). The aim of the present study was to deeply analyse the gross structure and the cellular arrangement of the vascular wall of small mesenteric arteries (SMA) from young and old rats and to analyse the morphological alterations of cell nuclei associated to ageing process.

Material and Methods: $3^{\text {rd }}$ branches of mesenteric artery (SMA) from 3 (young) and 22 (old) month-old male Sprague-Dawley rats were set up in a pressure myograph, fixed with paraformaldehyde $(4 \%)$ and stained with the

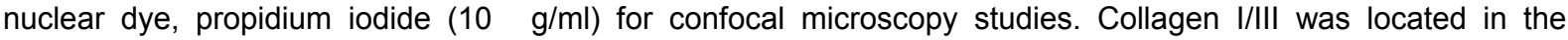
vascular wall by immunofluorescence.

Results: SMA from aged rats show increased external and internal diameter and cross sectional area as determined by pressure myography. Both the pressure myograph and the confocal microscope showed similar total wall thickness in SMA from young and old rats. However, an increase in media thickness $(P<0.05)$ was observed in arteries from old rats by confocal microscopy. The increased media thickness in arteries from aged rats was associated with an increase in the number of smooth muscle cells (SMC) layers, similar total number of SMC, decreased density of SMC and increased collagen deposition. The density of endothelial cells (EC) decreased but the luminal surface area increased by age. Thus, the total number of EC per vessel length remained unaltered by age. Total area of SMC nuclei was similar between age groups. However, total area of EC nuclei was significantly higher in old than in young rats. This increase in total area is due to a higher nuclei length and not to changes in nuclei width.

Discussion: 1) MRA from aged rats undergo a process of hypertrophic outward remodelling associated to changes in the media width and SMC density likely due to increased collagen deposition in this layer. 2) Ageing differently affect cellular morphology: SMC morphology remains unaltered whereas EC nuclei seem to be hypertrophied; the latter could explain, at least in part, the endothelial dysfunction reported in ageing.

(Supported by DGCYT (2003-01001) and Fundación Salud 2000) 


\title{
Session 4: Vascular biology: Endothelium - part 1
}

\author{
Abtsract \#: $4-1$ \\ HYPOXIA-INDUCED ENDOTHELIAL NITRIC OXIDE SYNTHASE UPREGULATION IN HUMAN UMBILICAL \\ VEIN ENDOTHELIAL CELLS
}

Østergaard L, U. Simonsen, H. Vorum, T. Ledet, M. Mulvany

Department of Pharmacology, University of Aarhus Wilhelm Meyers Alle, byg. 2408000 Århus C Denmark

Hypoxia-induced endothelial nitric oxide synthase upregulation in human umbilical vein endothelial cells L. Østergaard, U. Simonsen, T. Ledet, M. Mulvany Department of Pharmacology and Research Laboratory for Biochemical Pathology, University of Aarhus, Denmark It has previously been shown that the activity/stability of HIF is regulated, at least in part, by NO. Furthermore, the NOS II promoter region in mice and rats contains a HIF-1 response element (Jung F et al., Circ Res 2000; 86: 319-325) and the NOS III promoter in humans is activated by HIF-2 (J Biol Chem. 2003 Nov 21;278(47):46230-40) indicating a correlation between the two factors. The aim of the present study was to investigate the signalling pathways leading to the activation of inducible factor 1 (HIF-1) and endothelial nitric oxide synthase (eNOS) in hypoxia. Methods: Primary endothelial cells were isolated from human umbilical veins by perfusion with a $0,1 \%$ collagenase solution. The veins were incubated for 1 hour at $37 \& \# 730 ; C$ and then washed with DMEM to wash out the cells. After spinning down the cells were grown in gelatine-coated flasks for up to a maximum of 5 passages. The hypoxic incubation was carried out in an airtight chamber placed in a heating cabinet $(37 \& \# 730 ; \mathrm{C})$. The chamber was flushed with $\mathrm{N} 2$ until the $\mathrm{O} 2$ concentration was down to $5 \%$. The pressure of $\mathrm{O} 2$ was continuously monitored using an O2-probe (Unisense, Denmark). The protein contents (HIF-1 $\alpha$; , eNOS, Akt) were evaluated using immunoblotting. Proteins in cell homogenates were separated by reduced and denatured polyacrylamide gel electrophoresis (SDS-PAGE), blotted to a polyvinyldiflouride (PVDF) membrane and the selected proteins were visualized by incubation with the appropriate antibodies. For quantification immunoblotting for \&\#946;--actin were applied. Results: The stabilization of HIF-1 $\alpha$; was observed in hypoxic cell cultures after 30 minutes and continued for up to 24 hours. In the same cell homogenates a time-dependent up-regulation of eNOS and Akt protein content was seen. eNOS quickly reached a plateau and remained constant for the duration of the experiment ( 24 hours) whereas the amount of Akt protein continued to increase over the time period. Conclusions: The present results suggest hypoxia, through stabilization of HIF-1 $\alpha$; leads to an early and maintained up-regulation of Akt and eNOS, which would function as a protective mechanism against hypoxia-evoked vasoconstriction. 


\title{
Abtsract \#: $4-2$ \\ ISOLATED CORONARY ARTERIOLES EXHIBIT REACTIVE DILATION. ROLE OF HEMODYNAMIC FORCES, NITRIC OXIDE AND HYDROGEN PEROXIDE.
}

\author{
Koller A, Bagi Z
}

Department of Physiology, New York Medical College, Valhalla, New York 10595, USA Department of Pathophysiology, Semmelweis University, 1445-Budapest, Hungary

The role of metabolic factors derived from cardiac muscle in the development of reactive hyperemia following brief occlusions of the coronary circulation seems to be well established. However, the contribution of occlusion-induced changes in hemodynamic forces to reactive hyperemia is less known. We hypothesized that changes in intraluminal pressure $(P)$ and flow $(F)$, during and after release of an occlusion $(\mathrm{O} / \mathrm{R})$, via activating intrinsic mechanosensitive mechanisms, elicit the release of vasoactive factors resulting in reactive dilations of coronary arterioles. Thus, in isolated coronary arterioles (diameter: $88+/-8 \mathrm{~mm}$ ) changes in diameter to changes in $\mathrm{P}$ or $\mathrm{P}+\mathrm{F}$ during and after brief periods $(30,60$ and $120 \mathrm{~s})$ of $\mathrm{O} / \mathrm{R}$ of the cannulating tube were measured by videomicroscopy. In response to both types of O/R, diameters first decreased, then subsequently increased during occlusions. When only $P$ was changed (form $80-10-80 \mathrm{mmHg}$ ) after release of occlusion peak dilations increased as a function of the duration of occlusions. After establishing flow $(30 \mathrm{~mL} / \mathrm{min}$ ), O/R elicited changes in both $\mathrm{P}$ and $\mathrm{F}$ (form $80-10-80$ $\mathrm{mmHg}$ and 0 to $30 \mathrm{~mL} / \mathrm{min}$ ). In these conditions, not only the peak but also the duration of reactive dilation increased significantly as a function of the length of occlusions. The dilations during occlusions and the peak reactive dilations both in $\mathrm{P}$ and $\mathrm{P}+\mathrm{F}$ protocols were significantly reduced by the inhibition of NO synthase with L-NAME or by endothelium removal, whereas duration of the reactive dilations were reduced by L-NAME or by endothelium removal only in the P+F protocol. Also, in both protocols presence of catalase did not effect changes in diameter during occlusions, whereas significantly reduced the early phase of post-occlusion dilations and had less effect on the later phase of reactive dilations. Thus, mechanosensitive mechanisms that are sensitive to deformation, pressure, stretch and shear stress elicit release of $\mathrm{NO}$ and hydrogen peroxide resulting in reactive dilation of isolated coronary arterioles. We propose that these mechanisms contribute importantly to the development of reactive hyperemia and maintenance of diastolic coronary blood flow. (Supported by Hungarian NSRF OTKA, T-034779, and the NHLBI USA HL-46813, HL-43023) 
Abtsract \#: $4-3$

IS THE ENDOTHELIUM OSCILLATING DURING VASOMOTION?

Rahman A; Hughes A; Nilsson H; Aalkjaer C

Water and Salt Research Centre, Department of Physiology, Aarhus University and Department of Clinical Pharmacology, St. Mary's Hospital, University College London

Vasomotion is the oscillation of tone seen in all vascular beds investigated both under in vitro conditions and in vivo. The role of the endothelium for vasomotion is unclear. In this study we wanted to investigate whether the oscillation of tension is associated with oscillations of [Ca2+]i in the endothelial cells.

Isometric tension and $[\mathrm{Ca} 2+] \mathrm{i}$ was measured simultaneously in isolated rat mesenteric small arteries. $[\mathrm{Ca} 2+]$ i was measured with the fluorescent $\mathrm{Ca} 2+$ indicator Calcium Green using confocal microscopy and focusing on smooth muscle cells (SMC) or endothelial cells. To keep the arteries from moving the experiments were made with 1-7 uM wortmannin. Noradrenaline induced an oscillation of tension and SMC $[\mathrm{Ca} 2+]$ i which were in phase. Oscillations in endothelial $[\mathrm{Ca} 2+]$ i were either absent or of very low amplitude. Acetylcholine caused relaxation, increase of endothelial [Ca2+]i and decrease of SMC [Ca2+]i as expected. After blockade of the $\mathrm{Ca} 2+$ pump of the endoplasmic reticulum with cyclopiazonic acid, noradrenaline induced an oscillation of tension and SMC [Ca2+]i (in phase) with low frequency and high amplitude. This was associated with large oscillations in endothelial [Ca2+]i with the same frequency but in antiphase with the SMC. These oscillations disappeared after treatment with charybdotoxin and apamin or after treatment with nifedipine and after removal of the endothelium. The data suggest that in the absence of a functional endoplasmic reticulum a reciprocal interaction between oscillations in the endothelium and SMC can cause vasomotion. 


\title{
Abtsract \#: $4-5$ \\ CORONARY RESISTANCE ARTERY DYSFUNCTION IN RAT CARDIAC ALLOGRAFT IS REVERSED BY CYCLOSPORINE A TREATMENT
}

\author{
Moien-Afshari F; Choy JC; McManus BM; Laher I
}

Department of Pharmacology and Therapeutics \& Pathology, 2176 Health Sciences Mall, University of British Columbia, Vancouver, BC, V6T 1Z3, Canada

\begin{abstract}
Introduction: The mediators released by immune cells during the course of allograft rejection can increase the inducible isoform of nitric oxide synthase (iNOS) in vascular smooth muscle cells (SMCs). Once induced, iNOS can produce sufficient amounts of NO to cause sustained vasodilation of resistance arteries, which can lead to deterioration of coronary circulatory homeostasis and cause interstitial edema, and cardiac muscle contractile ability, and eventually ventricular failure. Increased levels of NO in arteries can also cause contractile dysfunction indirectly by decreasing the number of viable SMCs through induction of apoptosis. The host's immune system also damages SMCs in allograft tissue directly. Cyclosporine $A(C s A)$ is an immunosuppressive agent currently used for prevention of allograft rejection. CsA inhibits the production of interleukin-2 and other lymphokines by T-helper lymphocytes and therefore can decrease the excessive production of $\mathrm{NO}$ in transplant tissue. Background and purposes: A marked decline in vascular myogenic response occurs during the course of rat cardiac allograft rejection. Two important contributory features are an iNOS-catalyzed, NO-mediated vasodilation and a loss of smooth muscle function. In this study, we examined the effect of cyclosporine A immunosuppressive therapy on the alleviation of arterial dysfunction of coronary resistance arteries in allografts using pressure myography. Material and methods: Rats receiving heterotopic abdominal cardiac transplantation were treated with cyclosporine-A $(5 \mathrm{mg} / \mathrm{kg})$, Cremophore, or distilled water. Coronary septal arteries (internal diameter $200 \mathrm{~mm}$ ) were dissected from isograft (Lewis-Lewis) and allograft (Fisher-Lewis) rat hearts at day-21 post-transplantation and mounted on a pressure myograph. Pressure-induced vasoconstriction was measured before and after iNOS inhibition with aminoguanidine $(100 \mathrm{mM})$. Endothelium based (ACh-induced) and endothelium independent (SNP-induced) vasorelaxation were also recorded in each group. Results and discussion: Pressure-induced myogenic contraction was reduced in allograft coronary arteries at day-21 post-transplantation compared to matched isografts $(p<0.05)$. AG potentiated myogenic tone in allograft arteries but had no effect on untreated day-21 isograft vessels indicating the presence of iNOS based relaxation only in allograft vessels. Depolarization-induced vasoconstriction was lower in allograft- in compared to isograft arteries $(p<0.05)$. Cyclosporine A therapy also improved depolarization-induced constriction in allograft vessels compared to untreated groups $(p<0.05)$. Furthermore, Cyclosporine A therapy preserved endothelium-based and endothelium-independent vasorelaxation in allograft arteries at day-21 post-transplantation. In Conclusion, cyclosporine A immunosuppressive therapy has a significant effect in the alleviation of early endothelial and smooth muscle dysfunction in coronary allograft arteries.
\end{abstract}




\title{
Abtsract \#: 4-6 \\ HETEROGENEITY OF THE NEUROPEPTIDE Y (NPY) CONTRACTILE AND RELAXING RECEPTORS IN PENILE SMALL ARTERIES
}

\author{
Prieto D; Rivera de los Arcos L; Martínez P; Benedito S; García-Sacristán A; Hernández M
}

Departamento de Fisiología, Facultad de Farmacia, Universidad Complutense, 28040-Madrid, SPAIN

Neuropeptide Y (NPY) was initially suggested to play a role in detumescence on the basis of the rich NPY-peptidergic innervation found in penile erectile tissues; however, in vitro studies showed inconsistent contractile effects of the peptide in human corpum cavernosum, cavernous artery and penile veins (Andersson \& Wagner, Physiol. Rev., 75: 191, 1995). Moreover, NPY, considered as a vasoconstrictor peptide, increased intracavernous pressure and led to penile tumescence when injected intracavernously in rabbits (Kirkeby et al., J. Urol., 40: 270,1992). The aim of the present study was to investigate the distribution of NPY-immunorective (IR) nerves and the receptors involved in the effects of NPY upon electrical field stimulation (EFS)- and noradrenaline (NA)-elicited contractions in penile small arteries. Specimens of horse corpus cavernosum were processed for immunohistochemistry following the avidin-biotin-peroxidase complex method and second- and third-order branches of the deep penile artery were dissected and mounted in microvascular myographs for isometric tension recording. NPY-IR nerves were widely distributed in the erectile tissues with a particularly high density around penile small arteries. NPY and the NPY Y1 receptor agonist, [Leu31, Pro34]NPY, moderately enhanced contractions elicited by both EFS and NA, whereas the NPY Y2 receptor agonist NPY(13-36) potentiated responses to exogenous NA. In NA-precontracted arteries, NPY, peptide YY (PYY), [Leu31, Pro34]NPY and the NPY Y2 receptor agonist, N-acetyl[Leu28,31]NPY(24-36) elicited small contractions. NPY(13-36) contracted 8 out of 14 arteries tested. Human pancreatic polypeptide (hPP) evoked a biphasic response consisting of a relaxation followed by contraction. The long C-terminal fragment NPY(3-36) elicited moderate relaxations. The NPY Y1 receptor antagonist BIBP3226 $(0.3 \mu \mathrm{M})$ shifted to the right the concentration-response curves to both NPY and [Leu31, Pro34]NPY, but did not inhibit relaxations elicited by the NPY analogues. In the presence of the NPY Y2 receptor antagonist BIIE0246 $(0.3 \mu \mathrm{M})$, the contractions evoked by both $\mathrm{N}$-acetyl[Leu28,31]NPY (24-36) and NPY(13-36) turned into relaxations of the NA-precontracted arteries. Mechanical removal of the endothelium markedly enhanced contractions of NPY on NA-precontracted arteries, whereas blockade of neuronal voltage-dependent $\mathrm{Ca2}+$ channels did not alter NPY responses. These results suggest that NPY can elicit dual contractile/relaxing responses in penile small arteries through a heterogeneous population of NPY receptors. Potentiation of the contractions evoked by NA involve both NPY Y1 and Y2 receptors. A relaxation probably mediated by an atypical endothelial NPY receptor is also demonstrated, which could explain the erectogenic effects found for the peptide.

Supported by the Spanish Ministry of Science and Technology (SAF2002/02923) 


\title{
Abtsract \#: $4-7$ \\ SILDENAFIL RELAXES PENILE SMALL ARTERIES BY INCREASING THE EFFECTS OF BASALLY-RELEASED NITRIC OXIDE (NO)
}

\author{
Prieto D; Rivera de los Arcos L; Recio P; Hernández M; García-Sacristán A
}

Departamento de Fisiología, Facultad de Farmacia, Universidad Complutense, 28040-Madrid, SPAIN

Penile erection occurs when nitric oxide (NO) released from nerves and endothelium on sexual stimulation decreases penile vascular resistance by relaxing tonically constricted helicine arteries and corporal smooth muscle (Andersson \& Wagner, Physiol. Rev., 75: 191, 1995; Simonsen et al, J. Vasc. Res. 39: 283, 2002). By inhibiting cGMP-specific phosphodiesterase 5 (PDE5), sildenafil compensates for the reduced NO release, cGMP production and impaired penile perfusion in male erectile dysfunction (Goldstein et al., N. Engl. J. Med. 338: 1397, 1998). Whereas in corporal smooth muscle sildenafil mainly enhances NO-elicited relaxations, in penile resistance arteries it has a direct relaxant effect (Simonsen et al., 2002). The aim of the present study was to investigate the mechanisms of the sildenafil-induced relaxations in penile small arteries. Second- and third-order branches of the horse deep penile artery were and mounted in microvascular myographs for isometric tension recording. Sildenafil elicited potent relaxations of penile small arteries precontracted with phenylephrine (Phe), pD2 and maximum relaxation (Emax) being $7.68+/-0.04$ and $93.2+/-1.2 \%$ of the Phe-induced contraction $(n=31)$. Blockade of guanylate cyclase with $O D Q$, mechanical removal of the endothelium and inhibition of NO synthase with L-NOARG reduced sildenafil Emax to $40.7+/-2.9 \%(n=6), 27.9+/-5.5 \%(n=6)$ and $51.9+/-6.4 \%(n=7)$, respectively. Inhibitors of the cGMP-dependent (PKG) and the cAMP-dependent (PKA) protein kinases, $\mathrm{Rp}-8-\mathrm{Br}-\mathrm{PET}-\mathrm{cGMPS}$ and Rp-8-CPT-cAMPS, respectively, shifted to the right the relaxation curves of sildenafil (pD2 $7.54+/-0.06$ and $7.14+/-0.06, n=6, p<0.001$, in the absence and presence of Rp-8-Br-PET-cGMPS, and $7.86+/-0.08$ and 7.11/- 0.13, $n=5, p<0.05$, in the absence and presence of Rp-8-CPT-cAMPS). Raising extracellular $\mathrm{K}+$ to $30 \mathrm{mM}$ inhibited sildenadil Emax from to $93.7+/-1.7 \%$ to $66.8+/-9.4(p<0.05, n=6)$, whereas blockade of $\mathrm{Ca} 2+$ activated $\mathrm{K}+$ channels $(\mathrm{KCa})$ with either tetraethylammonium (TEA) or charybdotoxin shifted to the rifgt the sildenafil concentration-response curves (pD2 $7.74+/-0.09$ and $7.16+/-0.24, n=8, p<0.05$, in the absence and presence of TEA, and 7.85 $+/-0.05$ and $7.33+/-0.06, n=5, p<0.01$, in the absence and presence of charybdotoxin). The present results demonstrate a potent relaxant effect of sildenafil in penile resistance arteries due in part to cGMP accumulation and to the increased effects of basally-released endothelial NO acting through activation of PKG and KCa channels, although a PKA-mediated mechanism can not be excluded. Supported by grant $\mathrm{n}^{\circ}$ SAF2002/02923 from the Spanish Ministry of Science and Technology 


\section{Abtsract \#: $4-8$ \\ MECHANISMS OF ACETYLCHOLINE-INDUCED ENDOTHELIUM-DEPENDENT CONTRACTION IN RAT RENAL ARTERY}

Gao Yu-Jing; Lee Robert M.K.W.

Smooth Muscle Research Program and Department of Anesthesia McMaster University, 1200 Main Street West, Hamilton, Ontario, Canada L8N $3 Z 5$

In addition to endothelium-derived relaxing factor and hyperpolarizing factor, vascular endothelium also modulates underlying smooth muscle tone by releasing endothelium-derived contracting factor(s) (EDCF). The identity of the EDCF remains obscure. In this study, the involvement of reactive oxygen species in endothelium-dependent contraction of rat renal artery to acetylcholine (ACh) was investigated. ACh (10-5 $\mathrm{M})$ induced a transient contraction of rat renal artery with intact endothelium, but not in the artery with endothelium removed. Nitric oxide synthase inhibitor (e.g. NG-nitro-L-arginine) enhanced the contraction to ACh. Treatment with catalase greatly reduced the EDC to ACh, while the contraction to phenylephrine or to $\mathrm{KCl}$ was not affected by catalase. Cyclooxygenase inhibitor (diclofenac), thromboxane A2/prostaglandine $\mathrm{H} 2$ receptor antagonist (SQ 29548) abolished EDC. Thromboxane A2 synthase inhibitor (furegrelate) attenuated EDC. ACh enhanced the production of hydrogen peroxide (detected by Amplex Red) and thromboxane B2 (detected with ELISA kit), a metabolite of thromboxane A2, in the renal artery with intact endothelium. These results suggested that hydrogen peroxide is probably the mediator of EDC of rat renal artery in response to ACh. 


\title{
Abtsrat \#: $4-9$ \\ SIMULTANEOUS MEASUREMENTS OF FLOW AND VASODILATATION IN ISOLATED RAT MESENTERIC SMALL ARTERIES
}

\author{
Lopez-Valverde V(1), 2Damgaard L. 1Simonsen U.
}

1Department of Pharmacology, University of Aarhus, and 2Unisense A/S, 8000 Aarhus C, Denmark

The vascular endothelium has a pivotal role in sensing and transmitting alterations of wall shear stress to release vasoactive factors that affect the contractile state of smooth muscle and hence vessel diameter. In isolated vascular segments flow is often generated by establishing pressure differences, but only few attempts have been made to directly correlate increases in flow to vasodilatation. The purpose of the present study was to gain evidence for a direct relationship between flow and vasodilatation and study the underlying mechanisms. Rat mesenteric small arteries were suspended in a pressure myograph and vessel diameter measured simultaneously with flow registered by use of an ultra low flow unit consisting of a central hydrogen microsensor which gives changes in current when hydrogen is removed by solution passing through the sensor. The calibration of the flow sensor was performed with a syringe pump and responses were repeatable. Small mesenteric arteries were contracted with thromboxane analogue, $U$ $46619(0.1 \mu \mathrm{M})$, and after a stable contraction, flow was generated by differences of pressures between inflow and outflow pipettes. Flow generated by pressure differences of $10 \mathrm{mmHg}$ evoked vasodilatation and flows of, respectively, $5 \pm 3 \%$ and $10 \pm 3 \mu \mathrm{L} / \mathrm{min}$ and these parameters were enhanced by pressure differences of $80 \mathrm{mmHg}(26 \pm 3 \%$ and $44 \pm 8 \mu \mathrm{L} / \mathrm{min})$. Flow of $25 \mu \mathrm{Lmin}-1$ induced by a peristaltic pump induced vasodilatations comparable to those induced by pressure differences of $30 \mathrm{mmHg}(35 \pm 7 \mathrm{vs} 37 \pm 8$, $\mathrm{n}=5-7$, respectively). Endothelial cell removal abolished acetylcholine- and flow-evoked vasodilatation, but an inhibitor of nitric oxide (NO) synthase, NG, NG-assymmetric dimethyl-L-arginine (ADMA, $1 \mathrm{mM}$ ), abolished only flow-evoked dilatation. An inhibitor of guanylate cyclase, ODQ ( $3 \mu \mathrm{M})$ abolished vasodilatation induced by S-nitrosopenicillamine $(0.1-100 \mu \mathrm{M})$ and by flow, but did no change acetylcholine vasodilatation. In conclusion, the ultra low flow sensor allowed simultaneous measurements of flow and vessel diameter changes within a physiological range of flows and shows a direct relationship between flow and vasodilatation. Moreover, flow-evoked vasodilatation is endothelium-dependent and mediated by NO followed by activation of soluble guanylyl cyclase. 


\title{
Abtsract \#: $4-10$ \\ HIGH PRESSURE SELECTIVELY IMPAIRS FLOW-MEDIATED VASODILATATION IN RAT MESENTERIC SMALL ARTERIES
}

\author{
Christensen FH, Stankevicius E, Hansen T, Buus NH, Simonsen U
}

Department of Pharmacology, University Park 240, University of Aarhus, 8000 Aarhus C, Denmark

In renal hypertension decreased agonist-evoked release of nitric oxide (NO) was suggested to play a role for endothelial dysfunction, but it unclear whether flow-evoked and endothelium-derived hyperpolarizing factor (EDHF)-type vasodilatation are also affected. Therefore, the purpose of the present study was to investigate whether an acute increase of intraluminal pressure alters flow and agonist induced endothelium-dependent vasodilatation in rat mesenteric small arteries, and if treatment of renal hypertensive rats with the antioxidant tempol is able to reduce blood pressure and restore flow and agonist induced endothelium-dependent vasodilatation. Mesenteric small arteries were mounted in a pressure myograph, and pressure was raised to $50 \mathrm{mmHg}$. The vessel segment was contracted with the thromboxane analogue, U46619, and vasodilatation was induced either by increasing flow or by addition of acetylcholine. Superoxide was measured by confocal microscopy of vascular segments stained with dihydroethidium bromide. Incubation with an inhibitor of nitric oxide (NO) synthase, asymmetric dimethyl-L-arginine (ADMA, $1 \mathrm{mM}$ ) abolished flow-induced vasodilatation, but did not change acetylcholine-evoked vasodilatation. A transient pressure rise from 50 to $120 \mathrm{mmHg}$ for one hour almost abolished flow-induced vasodilatation, but it did not change acetylcholine-evoked vasodilatation. Dihydroethidium fluorescence signals were increased in vessel exposed to a pressure of $120 \mathrm{mmHg}$, indicating an increased production of superoxide in these vessels. Immunohistochemistry showed the presence of the 447 subunit of $N A D(P) H$ oxidase in the adventitia, and treatment with an inhibitor of $\mathrm{NAD}(\mathrm{P}) \mathrm{H}$ oxidases, apocynin, and a cell permeable superoxide dismutase mimetic, tempol, restored flow-induced vasodilatation in arteries exposed to high pressure. Tempol treatment of renal hypertensive rats significantly lowered systolic blood pressure (SBP) of renal hypertensive rats compared to vehicle-treated renal hypertensive rats. Flow-induced vasodilatation, which is mediated by NO, was lower in mesenteric arteries from renal hypertensive rats compared with normotensive rats, and was restored by treatment with tempol. Acetylcholine-evoked endothelium-derived hyperpolarizing factor (EDHF)-type vasodilatation of mesenteric arteries was not changed in renal hypertensive rats or by tempol treatment. The present study suggests that high pressure per se selectively inhibits flow-induced NO-mediated vasodilatation, probably through increased formation of superoxide derived from $N A D(P) H$ oxidase. This is also supported by the observation that tempol is able to lower SBP and correct the blunted flow-evoked NO-mediated vasodilatation in renal hypertensive rats. 


\title{
Abtsract \#: $4-11$ \\ CELLULAR MECHANISMS BY WHICH LOCAL PRESSURE INDUCES VASODILATION IN RAT SKIN: INVOLVEMENT OF POTASSIUM CHANNELS.
}

\author{
Garry A; Dumont O; Sigaudo-Roussel D; Saumet JL; Fromy B. \\ Laboratoire de Physiologie, UMR CNRS 6188, Angers, France.
}

\begin{abstract}
A pressure-induced vasodilation (PIV) was reported as a protective mechanism of the skin against pressure. Since the absence of PIV could be involved in the development of diabetic ulceration, the study of the underlying cellular mechanisms of PIV deserves further investigations. The opening of potassium (K+) channels in vascular smooth muscle cells contributes to endothelium-dependent vasodilation. As PIV is endothelium-dependent, we hypothesised that PIV depends at least partly on the opening of K+ channels. If so, we then aimed to determine the type of $\mathrm{K}+$ channels involved in PIV. To test this hypothesis, we first examined the involvement of $\mathrm{K}+$ channels in the vasodilation to local pressure applied at $11.1 \mathrm{~Pa} / \mathrm{s}$ in anaesthetised rats using tetraethylammonium (TEA), a non-specific $\mathrm{K}+$ channels blocker. The types of $\mathrm{K}+$ channels involved in PIV were then determined by the use of more specific blockers. In order to specify the site of $\mathrm{K}+$ channels involved in PIV, the endothelium-dependent and independent vasodilator responses were tested using iontophoretic delivery of acetylcholine (ACh) and sodium nitroprusside (SNP) respectively. PIV was reduced following TEA, charybdotoxin, iberiotoxin and glibenclamide administrations. The responses to ACh and SNP were reduced by iberiotoxin, whereas they were unchanged by glibenclamide. The present findings showed that large conductance calcium-activated $\mathrm{K}+$ channels (BK-Ca) and ATP-sensitive $\mathrm{K}+$ channels (K-ATP) channels play major roles in PIV. We suggest that endothelial nitric oxide (NO) and prostaglandin (PG) release lead to the opening of BK-Ca channels mainly via the NO/cGMP pathway and the K-ATP channels mainly via the PG/cAMP pathway to produce PIV through hyperpolarization of vascular smooth muscle cells.
\end{abstract}




\title{
Abtsract \#: $4-12$ \\ INVOLVEMENT OF ALPHA-1 INTEGRINS IN THE ACTIVATION OF AKT AND NO-SYNTHESIS AFTER FLOW (SHEAR STRESS) ACTIVATION OF VASODILATION IN RESISTANCE ARTERIES.
}

\author{
Dumont O, Laurent Loufrani, Dong You, Bernard Levy, Daniel Henrion.
}

CNRS UMR 6188, University of Angers, France.

Resistance arteries are the site of the earliest manifestations of many cardiovascular and metabolic diseases. Integrins are glycoproteins transmitting signals from the extracellular matrix to the cytoskeleton. Several integrins serving as mechanotransducer in endothelial cells migration might play a role in acute flow-mediated endothelial-dependent vasodilation (FMD). Flow (shear stress) is the main physiological stimulus for the vascular endothelium and its role in angiogenesis is well established. The alpha-1 integrin, which has a key role in angiogenesis, could be involved in FMD. Thus, we assessed the dilatory response to acute changes in flow in mesenteric resistance arteries (MRA) isolated in arteriographs. Flow-mediated dilation (FMD) in MRA was significantly decreased by anti-alpha-1 blocking antibodies. Similarly, in alpha1-integrin deficient mice (alpha1-/-) FMD was lower than in control mice (23.8 \pm 1 versus $11.4 \pm 0.6 \mu \mathrm{m}$ maximal dilation). Pressure- (myogenic tone) and agonist-induced contractions, as well as acetylcholine- and sodium nitroprusside-induced dilation, were not affected by the absence of alpha1-integrin. In control mice, alpha1-integrin was present in MRA endothelial cells where it co-immunoprecipitated with phosphorylated-Akt. In MRA from alpha 1-/- mice, endothelial NO-synthase expression was not affected but phosphorylated-Akt was lower, compared to alpha1 +/+ mice. Thus, alpha1 integrin might have a key role in flow (shear stress)-dependent vasodilation in the microcirculation by transmitting the signal to eNOS through activation of Akt. Due to the central role of flow (shear stress) activation of the endothelium in vascular disorders this finding opens new perspectives in the pathophysiology of the microcirculation. 


\title{
Abtsragt \#: $4-13$ \\ HYPOTENSION WITH NO CHANGE IN AORTIC REACTIVITY IN TRANSGENIC RATS OVEREXPRESSING ENDOTHELIAL NITRIC OXIDE SYNTHASE
}

\author{
NADAUD S, BOULANGER CM, MATHIEU E, GANTEN U, PAUL M, SIBONY M and SOUBRIER F
}

INSERM U525, Faculté de Médecine Pitié-Salpétrière, 91 Bd de l'hôpital, 75013 Paris, France INSERM U541, Hôpital Lariboisière, 41 Bd de la chapelle, 75010 Paris, France

INTRODUCTION: NOS3-derived NO participates in vascular function and structure regulation. Deleting NOS3 gene induces hypertension and accelerates the development of atherosclerosis whereas an increase of NO release, by enhancement of NOS3 activity or expression, prevents neointimal formation and improves impaired relaxation in atherosclerotic arteries. AIM OF THE STUDY: we aimed to determine the effect of NOS3 overexpression on vascular function under basal conditions by studying a line of transgenic rats (NOS3-Tg) bearing 8-10 copies of the human NOS3 gene. METHODS: Transgenic rats (Fisher 344) were generated using a $40 \mathrm{~kb}$ DNA fragment containing the entire human NOS3 gene flanked by $10 \mathrm{~kb}$ of its 5'-promoter region and $10 \mathrm{~kb}$ of its 3'-untranslated region. Transgene copy number was determined using Southern-blots. Human and rat NOS3 expression was studied at the mRNA level using real-time PCR and northern-blots and at the protein level using western-blots, immunohistochemistry and total calcium-dependent NOS activity measurement. Vascular reactivity was evaluated on aortic rings. Aortic CGMP levels were determined by RIA. Arterial blood pressure was measured under pentobarbital-anesthesia by inserting a catheter in the common carotid artery. RESULTS: Total (rat and human) NOS3 mRNA levels were increased by $50 \%$ in NOS3-Tg rats (real-time PCR and northern-blots). Total NOS3 protein levels were increased 12 times (calcium-dependent NOS activity, western-blots), indicating modifications in NOS3 protein translation or degradation in transgenic animals. This high level of expression was confirmed by immunohistochemical staining of aorta for NOS3. Aortic cGMP levels were doubled in NOS3-Tg animals suggesting an increase in endothelial NO production. NOS3-Tg rats display an arterial pressure reduced by $15 \%$ as compared to wild-type animals (WT). Contractions to $\mathrm{KCl}$, phenylephrin and NOS inhibitor were not modified. No difference was detected between NOS3-Tg and WT animals in acetylcholine or inomycin-induced endothelium-dependent relaxation or in nitroprussiate (SNP)-induced endothelium-independent relaxation. However, acetylcholine relaxation in NOS3-Tg aortic rings seems to be partly dependent on reactive oxygen species $(\mathrm{H} 2 \mathrm{O} 2)$ release, as it is decreased in the presence of the antioxidant $\mathrm{N}$-acetylcysteine. CONCLUSION: These results indicate that in rat, high levels of NOS3 do not induce modifications of aortic reactivity but are accompanied by a decrease in blood pressure. 


\title{
Abtsract \#: $4-14$ \\ ABNORMALITIES IN SKIN MICROVASCULAR REACTIVITY ARE ASSOCIATED WITH INCREASED CARDIOVASCULAR RISK AND ELEVATED ALBUMIN EXCRETION RATE.
}

\author{
Strain WD; Chaturvedi N; Bulpitt CJ; Rajkumar C; Shore AC*
}

Imperial College School of Medicine, Norfolk Place, London, W2 1PG, UK *Peninsular Medical School (Exeter), Barrack Road, Exeter, EX2 5AX

Background: Elevated albumin excretion rate (AER) independently predicts total and cardiovascular mortality in a variety of conditions, although the exact mechanisms are unknown. These associations may indicate that AER is a marker of as yet unmeasured risk factors, or that AER is specifically an index of microvessel damage in other vascular beds, and that microvascular damage plays a key role in the development of cardiovascular disease and in the abnormal handling of albumin by the kidney. We aimed to explore associations between skin microvascular functional response to an ischaemic stimulus with cardiovascular risk factors and with AER to determine whether derangements seen in the renal microvascular bed were mirrored elsewhere. Methods: Laser Doppler fluximetry of skin vessels was used to study associations with risk factors and renal damage (AER calculated from a timed overnight urine collection) in 188 people without diabetes and 117 individuals with diabetes. Flow in response to arterial occlusion (ischaemia) was measured. Results: Three distinct patterns of post ischaemic peak flow were observed: 1) gradual rise to peak (normal) 2) non-dominant early peak and 3) dominant early peak. Those with a dominant early peak were more likely to have diabetes $(p=0.01)$, hypertension $(p=0.001)$ and obesity $(p<0.001)$, and had a higher AER (12.6 (95\% Cl 7.8-20.2) micrograms/min vs. 7.2 (5.5-9.5) micrograms/min in the non-dominant peak group and 3.7 (3.2-4.1) micrograms/min in the normal group; $p<0.001)$. In multivariate analysis this elevation in AER across the three groups could not be accounted for by conventional cardiovascular risk factors $(p<0.001)$. The association between peak response curve morphology and AER was also observed in analyses stratified by diabetes status and the use of antihypertensive therapies (no diabetes $p<0.001$; with diabetes $p=0.004$; no antihypertensives $p=0.002$; no diabetes and no antihypertensives $p=0.009$ ). Discussion: A rapid peak flow response following ischaemia is associated with an elevated AER and increased cardiovascular risk. This may represent shared mechanistic pathways, causative or consequential changes in the microvasculature. 


\title{
Abtsract \#: $4-15$ \\ ENDOTHELIUM-DEPENDENT HYPERPOLARIZATION IN DILATOR RESPONSES OF MYOGENICALLY ACTIVE MOUSE CREMASTER MUSCLE ARTERIOLES.
}

\author{
Potocnik SJ; Triggle CR; McSherry I; Dora K; Kotecha N; Hill MA.
}

School of Medical Sciences, RMIT University, Melbourne, Australia; Department of Pharmacy and Pharmacology, University of Bath, Bath, England; and Department of Physiology and Pharmacology, University of New South Wales, Sydney, Australia.

The component of acetylcholine-induced vasodilatation that is not blocked by combined cyclooxygenase and nitric oxide synthase inhibition is generally considered to be mediated via endothelium-derived hyperpolarizing factor (EDHF). While the nature of EDHF is yet to be fully characterized its activity is thought to be dependent on both small (SKCa) and intermediate (IKCa) conductance Ca2+-sensitive $\mathrm{K}+$ channels as shown by the effectiveness of a combination of apamin and charybdotoxin (or TRAM-34) in blocking the EDHF response. Despite the apparent importance of EDHF in small blood vessels few studies have been performed in myogenically active arterioles. This is somewhat surprising given the likelihood that resting membrane potential may influence vascular responsiveness and that myogenically active arterioles typically have resting membrane potentials in the order of $-30--40 \mathrm{mV}$ at physiological intraluminal pressures. Further, differences may exist between agonist-induced and myogenic tone when used in mechanistic studies of vasodilators. Studies were conducted in cannulated mouse cremaster muscle arterioles (passive diameter $72 \pm 0.6 \mathrm{uM}$ ) at $60 \mathrm{mmHg}$ under no flow conditions. Resting membrane potential under these conditions was measured at $-41.7 \pm 1.2 \mathrm{mV}$ using glass microelectrodes. Myogenic activity was demonstrated by examining vasoconstrictor responses to step increases in intraluminal pressure. Acetylcholine concentration-response curves showed a significant shift to the right in the presence of indomethacin (10 uM) and L-NAME (100 uM) although maximal vasodilatation could still be achieved suggesting a significant EDHF component. Consistent with the requirement for both IKCa and SKCa channels the residual dilator response was totally inhibited by a combination of apamin $(1 \mathrm{uM})$ and charydotoxin $(0.1 \mathrm{uM})$. Apamin alone was ineffective in blocking the acetylcholine response above that caused by indomethacin and L-NAME. Charybdotoxin, itself, caused vasoconstriction presumably through an action on smooth muscle large conductance (BKCa) channels as shown by the action of the selective BKCa inhibitor iberiotoxin. Collectively the data support the involvement of IKCa and SKCa in the EDHF response of arterioles held under conditions of physiological intralumenal pressures and resting membrane potentials. 


\title{
Abtsract \#: $4-16$ \\ EVIDENCE FOR THE INVOLVEMENT OF VPAC1 AND VPAC2 RECEPTORS IN PRESSURE-INDUCED VASODILATATION IN RODENTS.
}

\author{
Fizanne L; Sigaudo-Roussel D; Saumet JL; Fromy B.
}

UMR-CNRS 6188, Laboratory of Physiology, University of Medecine, rue haute de Reculée, 49045 Angers, France.

\begin{abstract}
A transient increase in skin blood flow in response to an innocuous local pressure application, defined as pressure-induced vasodilatation (PIV), delays the occurrence of ischaemia, suggesting a protective feature against applied pressure. The PIV response depends on capsaicin-sensitive nerve fibres and calcitonin gene-related peptide (CGRP) has been shown to be involved. In these fibres, CGRP coexists with pituitary adenylate cyclase-activating polypeptide (PACAP). Three distinct receptors mediate the biological effects of PACAP: VPAC1 and VPAC2 receptors binding with the same affinity for PACAP and vasoactive intestinal peptide and PAC1 receptors showing high selectivity for PACAP. Because the receptors are widely expressed in the nervous system and in the skin, we hypothesized that at least one of them is involved in PIV development. To verify this hypothesis, we used [D-p-Cl-Phe(6),Leu(17)]-VIP (nonspecific antagonist of VPAC1/VPAC2 receptors), PG 97-269 (antagonist of VPAC1 receptors), PACAP(6-38) (antagonist of VPAC2/PAC1 receptors) and Max.d.4 (antagonist of PAC1 receptors) in anaesthetized rodents. The blockade of VPAC1/VPAC2, VPAC1 or VPAC2/PAC1 receptors eliminated the PIV response, whereas PAC1 blockade had no effect, demonstrating an involvement of VPAC1/VPAC2 receptors in PIV development. Moreover, endothelium-independent and -dependent vasodilator responses were unchanged by the VPAC1/VPAC2 antagonist. Thus, the absence of a PIV response following VPAC1/VPAC2 blockade cannot be explained by any dysfunction of the vascular smooth muscle or endothelial vasodilator capacity. The involvement of VPAC1/VPAC2 receptors in the development of PIV seems to imply a series relationship in which each receptor type (CGRP, VPAC1, VPAC2) is necessary for the full transmission of the response.
\end{abstract}




\title{
Abtsrat \#: $4-17$ \\ CA2+-ACTIVATED K+ CHANNEL BLOCKERS INHIBIT NITRIC OXIDE RELEASE AND RELAXATION IN RAT SUPERIOR MESENTERIC ARTERY
}

\author{
Stankevicius E, Lopez V, Rivera L, Hernanz R, Mulvany MJ, Simonsen U
}

Department of Pharmacology, University of Aarhus, 8000 Aarhus C, Denmark

The purpose of the present study was to investigate the role of $\mathrm{Ca} 2+-$ acitvated $\mathrm{K}+$ channels in acetylcholine-evoked nitric oxide (NO)-mediated vasorelaxation. Direct measurements were made of NO concentration, endothelial cell calcium, and wall tension in rat superior mesenteric artery suspended in microvascular myographs in vitro. In noradrenaline-contracted arteries, the relaxation to acetylcholine (10 \&\#956;M) was reduced, but not eliminated, in the presence of an inhibitor of NO synthase, asymmetric dimethylarginine (ADMA, 0.1-1 $\mathrm{mM}$ ) and indomethacin $(3 \mu \mathrm{M})$, a response which might be ascribed to endothelial derived hyperpolarizing factor (EDHF). However, our evidence suggests an involvement of NO. First, there was still a residual increase in NO in the presence of ADMA and indomethacin. Second, further addition of oxyhaemoglobin $(10 \mu \mathrm{M})$ reversed the residual increases in NO concentration and also relaxation. Third, residual responses were abolished by the combination of blockers of small-conductance $\mathrm{Ca} 2+$-activated $\mathrm{K}+$ channels, apamin $(0.5$ \&\#956;M) and of intermediate- and large-conductance Ca2+-activated $\mathrm{K}+$ channels, charybdotoxin (0.1 \&\#956;M). Fourth, endothelial cell resting membrane potential (ca. $-42 \mathrm{mV}$ ) was hyperpolarized $(7 \mathrm{mV})$ by acetylcholine, and the hyperpolarization was abolished by apamin plus charybdotoxin. In conclusion, our findings suggest endothelial cell $\mathrm{Ca} 2+-$ activated $\mathrm{K}+$ channels are involved in acetylcholine-evoked $\mathrm{NO}$ release in rat superior mesenteric artery. Moreover, the inhibitory effect of oxyhaemoglobin suggests responses, which might be ascribed to EDHF, can in part be explained in terms of residual NO release. 


\section{Abtsrat \#: $4-18$ \\ EARLY ENDOTHELIAL DYSFUNCTION SEVERELY IMPAIRS SKIN BLOOD FLOW RESPONSE TO LOCAL PRESSURE APPLICATION IN STREPTOZOTOCIN-DIABETIC MICE}

Demiot Claire, Dominique Sigaudo-Roussel, Bérengère Fromy, Audrey Koïtka, Georges Lefthériotis, Pierre Abraham and Jean Louis Saumet.

Laboratoire de physiologie, UMR CNRS 6188, Faculté de médecine d'Angers, 49045 Angers cedexFrance-

Pressure-induced vasodilation (PIV) is a mechanism where skin blood flow increases in response to progressive locally applied pressure. Skin blood flow in response to applied pressure, early decreased in diabetic patients due to vascular and/or neural impairment. This study was designed to determine the effect of vascular changes on PIV in 1-week streptozotocin-diabetic mice. We assessed cutaneous microvascular response to local increasing pressure application measured by laser Doppler flowmetry (LDF), endothelium-dependent and -independent vasodilation by iontophoretic delivery of acetylcholine and sodium nitroprusside, sciatic motor nerve conduction velocity and morphometry. In control mice, LDF increased $34 \%$ from baseline to $0.2 \mathrm{kPa}$ external pressure, showing PIV response. In contrast, diabetic mice had no LDF increase in response to progressive external pressure. Moreover, following iontophoretic delivery of acetycholine, endothelium-dependent vasodilation was largely attenuated in diabetic mice $(25 \%)$ compared to control mice (81\%) whereas vasodilation to sodium nitroprusside was not different between groups. Nerve function as assessed by sciatic nerve conduction velocity and morphometry did not differ between groups. These findings point out the fact that PIV response seems to be highly sensitive to endothelial NO levels and therefore endothelial impairment by itself is sufficient to severely alter the PIV response. We suggest that PIV could be a sensitive indicator of endothelium alteration occurring during diabetes. Pressure-induced vasodilation suppression could favour diabetic complications such as diabetic foot ulcers. Detecting and preventing early vascular dysfunction could delay the apparition of diabetic neuropathy and limit diabetic complications. Further experiments are needed to explore the individual involvement of each biochemical pathway in PIV disappearance. 


\title{
Abtsrat \#: $4-19$ \\ IMPAIRED PRESSURE-INDUCED VASODILATION AT THE FOOT IN YOUNG ADULTS WITH TYPE 1 DIABETES
}

Koïtka A ; Abraham P ; Bouhanick B ; Sigaudo-Roussel D ; Demiot C ; Saumet JL

laboratoire de physiologie et explorations vasculaires, Centre Hospitalier Universitaire, 4 rue larrey, f-49033 Angers Cédex, france

\begin{abstract}
Vascular and neurological mechanisms are both likely to be involved in foot ulcer. We recently reported a pressure-induced vasodilation (PIV), relying on unmyelinated afferent excitation. We previously found that cutaneous blood flow in response to locally applied pressure might be impaired in diabetic patients because of the combined effects of low cutaneous temperature and alterations in microcirculatory function. Therefore, we aimed to: analyse whether at a relatively high cutaneous temperature PIV is present in Type 1 diabetes mellitus, and assess endothelial-dependent vasodilation and endothelium-independent vasodilation. We measured cutaneous blood flow using laser Doppler flowmetry on the head of the first metatarsus in response to applied pressure at $5.0 \mathrm{~mm} \mathrm{Hg}$.min-1 in warm conditions $\left(29.5+/-0.2^{\circ} \mathrm{C}\right)$. Responses to iontophoresis of acetylcholine (endothelium-dependent) and sodium nitroprusside (endothelium-independent) were measured using laser Doppler flowmetry in the forearm. The data indicate that PIV exists at the foot level in normal subjects, whereas it was not found in diabetic patients. The non-endothelial-mediated response to sodium nitroprusside was preserved, whereas the endothelial-mediated response to acetylcholine was impaired in the diabetic patients. These findings might be relevant to the high prevalence of foot ulcer that occurs in diabetic patients.
\end{abstract}




\title{
Abtsrat \#: $4-20$ \\ ENDOTHELIAL EPOXYEICOSATRIENOIC ACIDS ACT ON SMOOTH MUSCLE, NOT ENDOTHELIUM, TO RELAX AND HYPERPOLARIZE BOVINE CORONARY ARTERIES
}

\author{
Campbell WB; Falck JR; Gauthier KM.
}

Medical College of Wisconsin, 8701 Watertown Plank Road, Milwaukee, WI, 53226 USA and University of Texas Southwestern Medical Center, 5323 Harry Hines BIvd, Dallas, TX,75235 USA

Endothelium-dependent hyperpolarizations and relaxations to bradykinin are mediated by endothelium-derived hyperpolarizing factors (EDHFs). In bovine coronary arteries, the cytochrome P450 (CYP) metabolites of arachidonic acid, the epoxyeicosatrienoic acids (EETs), function as EDHFs. The EET analog 14,15-epoxyeicosa-5Z-enoic acid (14,15-EEZE) inhibits relaxations to 14,15-, 11,12-, 8,9and 5,6-EET but not sodium nitroprusside, NS1619, bimakalim or iloprost. 14,15-EEZE acts as a selective EET antagonist. In the presence of L-nitroarginine (L-NA) and indomethacin (INDO), bradykinin and arachidonic acid cause concentration- related relaxations that are inhibited by $14,15-E E Z E$, the CYP inhibitor miconazole and the CYP2J2 inhibitor terfenadone. The CYP2C8/9 inhibitors sulfaphenzole, retinoic acid, $\mathrm{N}$-isopentanyl-sulfaphenazole or tienilic acid were without effect. Thus, endothelial EETs produced by CYP2J2, but not CYP2C8, mediate the non-NO, non-PG relaxations to bradykinin. It has been suggested that EETs act on the endothelium to release a factor, possibly potassium that mediates bradykinin-induced relaxations. However, EETs also relax and hyperpolarize smooth muscle. We developed a bioassay to determine the cellular site of action of the EETs that mediates relaxation. An L-NA/INDO-treated donor artery with endothelium was perfused in series with a detector artery without endothelium. Diameter of the detector vessel was measured to detect release of dilators from the donor. Addition of bradykinin to a L-NA/INDO-treated donor caused dilation of the detector artery indicating the release of a non-NO, non-PG transferable dilator. Addition of 14,15-EEZE to the donor did not alter bradykinin-induced dilation of the detector indicating that EETs do not act on the endothelium. Addition of 14,15-EEZE to the donor did not inhibit dilation to 14,15-EET added directly to the detector. In contrast, 14,15-EEZE added to the detector blocked the dilations to bradykinin added to the donor. Thus, 14,15-EEZE blocked the action of the transferable dilator indicating EETs act on the smooth muscle. If bradykinin was given directly to the detector, it was without effect in the presence or absence of 14,15-EEZE. These studies indicate that EETs mediate the non-NO, non-PG relaxations to bradykinin and exert their action on smooth muscle and not endothelium. EET synthesis is by a CYP2J2 and not CYP2C8/9. Sulfaphenazole is not an inhibitor of EET synthesis in coronary arteries. 


\title{
Abtsrat \#: $4-21$ \\ THE ORGANIC NITRATES: NO FORMATION, VENOSELECTIVITY \& OXYGEN DEPENDENCE
}

\author{
Mark R. Miller \& Roger M. Wadsworth
}

Department of Physiology and Pharmacology, University of Strathclyde, John Abuthnott Building, 27 Taylor Street,Glasgow G4 ONR, U.K.

The organic nitrates, such as glyceryl trinitrate (GTN), have been used in the treatment of angina for over 100 years, yet their mechanism of action via nitric oxide (NO) formation has yet to be fully established. Their success as anti-anginal agents may be due to their selectivity for veins over arteries in vivo, yet in vitro findings are often contradictory. This study investigates the NO generation using a direct measurement of NO over the same time course as vasodilatation. We also explore the arteriovenous selectivity of nitrates in vitro and whether this selectivity is affected by lowering oxygen concentrations to that of the venous system in vivo (PO2 $40 \mathrm{mmHg}$ ). Rat superior mesenteric arteries (SMA) and renal vein (RV) were isolated and mounted in a wire myograph. Nitric oxide was measured using an NO microelectrode positioned within the vessel lumen. Vasodilator responses to GTN $(0.01-30 \mathrm{mM})$ were investigated in precontracted blood vessels bathed in Kreb's buffer under different oxygen concentrations (PO2 $=35-200 \mathrm{mmHg}$ ). GTN produced a dose-dependent vasodilatation in the SMA. This relaxation was accompanied by the production of nanomolar concentrations of $\mathrm{NO}$ over the same dose range $(5.62 \pm 1.68$ $\mathrm{nM} \mathrm{NO}$ at $10 \mathrm{mM} \mathrm{GTN}$ ). When bubbled with 95\% O2/5\% CO2 (PO2 > $200 \mathrm{mmHg}$ ), GTN was found to be 10-100 fold less potent in the RV than the SMA. Other veins (mesenteric, portal \& femoral) showed similar weak responses to GTN. Bubbling with $0-5 \%$ O2, lowered oxygen concentrations in the myograph to $35-55 \mathrm{mmHg}$, within the range seen in the venous system in vivo. This oxygen concentration had no effect on the venodilator responses to the cAMP-dependent agonist, isoprenaline, the spontaneous NO generator, diethylamine NONOate, or endothelial-derived NO generated with carbachol. Similarly, lowering oxygen concentration had no effect on venodilator responses to GTN. This is the first time that GTN has been shown to cause vasodilatation through the release of nanomolar concentrations of NO using a direct measurement of NO simultaneously with vasodilatation. GTN was found to be more potent in arteries than veins in vitro. Lowering oxygen levels to mimic that of the venous system in vivo did not affect the arteriovenous selectivity of GTN in vitro, demonstrating that this set-up and protocol is a suitable way to investigate the mechanism of action of the organic nitrates. This work is supported by the British Heart Foundation (PG/02/129/14604). 


\author{
Abtsract \#: $4-22$ \\ BARRIER FUNCTION OF ARTERIOLAR AND VENULAR ENDOTHELIUM STUDIED IN VITRO: \\ PROTECTION \\ BY HYDROXYETHYLATED RUTOSIDES (HR) AGAINST DAMAGE INDUCED BY ACTIVATED BLOOD \\ COMPONENTS.
}

Nees S; Weiss DR; Heilmeier B

Dept. of Physiology, University of Munich, Schillerstr. 44, 80336 Munich, Germany

\begin{abstract}
Introduction: In situ observations suggest that recruitment of the immune defence occurs specifically at the venular endothelium of inflamed body areas. The extent to which the arteriolar endothelium may also respond to such inflammatory conditions is unknown. Purpose: Comparison of interactions between selected activated blood components with cultured arteriolar or venular endothelial cells (AEC, VEC) under defined conditions in vitro. Methods: Microscopically and immunologically identified postcapillary venules and arterioles were obtained by isopycnic centrifugation of guinea pig hearts that were disintegrated by perfusion with collagenase-dispasel-trypsin (1:0.1:0.1mg/ml). Fully differentiated AEC or VEC were purified by scratching and homo-genous monolayers cultures were established on porous filters in media containing adult guinea-pig serum. The hydraulic conductivity of these layers was measured and cellular interactions were followed with video time-lapse microscopy or scanning electron microscopy. Results: The hydraulic conductivity of VEC layers $(8 \times 10-6 \mathrm{~cm} / \mathrm{s} / \mathrm{cmH} 2 \mathrm{O})$ was not influenced by thrombin $(1 \mathrm{U} / \mathrm{ml})$ and/or platelets $(105 / \mathrm{ml})$, but was 3 - to 4 -fold increased in the presence of PMN $(104 / \mathrm{ml})$, which could be prevented by the presence of antioxidants or HR $(100 \mu \mathrm{M})$. Simultaneous exposure to thrombin, platelets and PMN or to the supernatant of this suspension caused prompt contraction of VEC, a characteristic reorganization of the F-actin filaments throughout the cytoplasm and a dramatic 16-fold increase in hydraulic conductivity. This in situ highly pathogenetic endothelial damage was prevented by addition of acetylsalicylic acid $(100 \mu \mathrm{M})$ or $\mathrm{HR}(100 \mu \mathrm{M})$ to the blood cell suspension prior to its application to VEC, but only by HR when the damage was induced by the activated supernatant. F-actin dissolved completely to monomeric G-actin in the presence of HR. The basal motility of VEC-adherent PMN $(1.15+/-0.4 \mu \mathrm{m} / \mathrm{min})$ was increased considerably by the activated supernatant $(6.5+/-1.2 \mu \mathrm{m} / \mathrm{min})$, whereas HR $(100 \mu \mathrm{M})$ caused complete detachment and inactivation of the activated PMN within $20 \mathrm{~min}$. In contrast, the hydraulic conductivity of AEC $(0.7 \times 10-6 \mathrm{~cm} / \mathrm{s} / \mathrm{cmH} 2 \mathrm{O})$ was not affected by activated blood components and was not influenced by HR. F-actin was only scanty in AEC and, due to the apparent absence of ELAMs, PMN did not firmly attach to these arteriolar endothelial cells. Conclusion: Thrombin-activated platelets cooperate with PMN to release oxidants and unknown eicosanoids, which are highly effective in opening the immunologically important endothelial barrier between the blood and inflamed tissues in local venules, but not arterioles. These effects can be accompanied pathophysiologically by spreading inflammatory reactions. Medication with ASS and HR appears to be a promising and practical prophylactic therapy.
\end{abstract}




\title{
Session 5: Vascular biology: Endothelium - part 2
}

\author{
Ahtsract \#: $5-1$ \\ DES-ACYL GHRELIN AND GHRELIN EVOKE POTENT VASODILATATION IN THE RAT MESENTERIC \\ VASCULAR BED: SELECTIVE ACTIVATION OF KCA CHANNELS ON ENDOTHELIAL CELLS. (NOTE THIS \\ A REVISED VERSION OF THE /TEXT OF THE ABSTRACT - RESUBMITTED AS DIRECTED) PLEASE \\ Moazed $\mathrm{B}^{*}$, Tang, $\mathrm{G}^{* *}$, Liu $\mathrm{P}^{*}$, Moien-Afshari $\mathrm{F}^{* * *}$, Washio $\mathrm{H}^{* * *}$, Laher $\mathrm{I}^{* * *}$, Triggle $\mathrm{CR}+$, McNeill $\mathrm{JR}^{*}$, \\ Wang $\mathrm{R}^{\star *}$, Gopalakrishnan V*.
}

Departments of Pharmacology* \& Physiology**, College of Medicine, University of Saskatchewan, Saskatoon, SK., S7N 5E5, Department of Pharmacology \& Therapeutics ${ }^{* * *}, 2176$, Health Scinces Mall, University of British Columbia, Vancouver, BC., V6T 1Z3, and th

Revised version of abstract is resubmitted; please delete the earlier version. Ghrelin and des-acyl ghrelin are peptides released from the stomach and are present in the systemic circulation. Ghrelin promotes growth hormone release (EC50 $1.5 \mathrm{nM}$ ) and increased food intake by binding to the growth hormone secretagogue (GHS) receptor whereas des-acyl ghrelin is devoid of GHS activity (EC50 $3.5 \mu \mathrm{M}$ ). While the vasodilator responses to ghrelin have been described in earlier studies, the mechanism of action has not been explored. Therefore, we determined the responses to both ghrelin and des-acyl ghrelin in the phenylephrine (PE) constricted perfused rat mesenteric vascular bed (MVB) maintained at a constant flow rate of $5 \mathrm{ml} / \mathrm{min}$. We demonstrate that low concentrations of both ghrelin and des-acyl ghrelin (EC50 $54 \pm$ $12 \mathrm{fM}$; Emax $34 \pm 15 \%$ ) evoke vasodilatation in comparison to the responses evoked by acetylcholine (EC50 $3 \pm 1 \mathrm{nM}$; Emax $92 \pm 4 \%$ ) in the PE constricted MVB. The vasodilatation was abolished in endothelium-denuded preparations or in endothelium-intact preparations exposed to either calcium activated potassium channel $(\mathrm{KCa})$ blockers or a depolarizing stimulus. While KATP channel blockade or a combination of nitric oxide synthase and cyclooxygenase inhibition had no effect, the vasodilator responses were abolished in the presence of a combination of charybdotoxin and apamin or ouabain and $\mathrm{Ba} 2+$. In cultured (3rd passage) rat mesenteric artery endothelial cells $(E C)$, both peptides bound to a single high affinity specific binding site ([125I] ghrelin Kd 256 pM) and evoked increases in [Ca2+]i that were attenuated by 2-aminoethoxyphenyl borate $(5 \mu \mathrm{M})$, an InsP3 selective antagonist. In freshly isolated EC subjected to culture for $<12 \mathrm{hr}$, both peptides (100 pM) enhanced KCa currents, and hyperpolarization, an effect that was attenuated by inclusion of a combination of apamin and charybdotoxin. The GHS antagonist, [D-Lys3]-GHRP-6 (10 nM), per se evoked vasodilatation and increased $[\mathrm{Ca} 2+] \mathrm{i}$ in EC. Both ghrelin and des-acyl ghrelin (100 pM) hyperpolarized vascular smooth muscle (between -12 and $-16 \mathrm{mV}$ ) in intact mesenteric arterioles mounted in a pressure myograph. These data indicate that the vasodilatation evoked by ghrelins results from endothelium-dependent hyperpolarization subsequent to the activation of endothelial receptors that are distinctly different from the classical GHS receptor. Our data suggest that, besides energy homeostasis, ghrelin and des-acyl ghrelin are potent vasodilators that act via nitric oxide and prostanoid independent mechanism, possibly through selective activation of endothelium-dependent hyperpolarization and causing relaxation of vascular smooth muscle cells. (Supported by Grants from the CIHR and the H\&SF to VG, RW and IL). 


\title{
Abtsrat \#: $5-2$ \\ ESTROGEN ENHANCED FLOW MEDIATED DILATION AND MYOGENIC TONE IN SMALL MESENTERIC ARTERIES OF THE BETA-ESTROGEN RECEPTOR KNOCKOUT MOUSE (BETA-ERKO)
}

\author{
Douglas G 1; Cruz M N 2; Gustafsson J A 3; Kublickiene K 2; Poston L 1
}

1 Maternal and Fetal Research Unit St Thomas\&\#8217;s Hospital, Kings College London, London, SE1 7EH, 2 Dept. of Obstetrics and Gynaecology and 3 Dept of Medical Nutrition, Karolinska Institutet, Huddinge Hospital, 14186 Stockholm.

Introduction and Background. Estrogens are known to improve flow mediated dilation and reduce myogenic tone in animal models. The mechanism behind these effects is poorly understood including the relative roles of the different estrogen receptors (ER alpha and beta). Aims: The aim of this study was to characterise the consequence of the loss of ER-beta on estrogen enhanced flow responses and myogenic tone in the resistance blood vessels from ER beta knock out mice (beta-ERKO) mice and wild type (WT) littermates. Methods: Mesenteric arteries ( 185 microm diameter) from male and female beta-ERKO mice and WT littermates were dissected and mounted on a pressure myograph. Flow mediated dilation $(9-104 \mathrm{~mL} / \mathrm{min})$ was assessed in norepinephrine pre-constricted arteries before and after incubation with 17beta-estradiol or, in different vessels, in the presence of the selective ER alpha agonist PPT (3h; 10nM). Pressure-induced myogenic tone $(20-100 \mathrm{mmHg})$ before and after incubation with 17b-estradiol was also studied. Results: Flow-mediated dilation increased after incubation with 17beta-estradiol in WT and beta-ERKO females and WT males [5,08 +/- 2,80 to 47.1 +/- 15.2, 15,62 +/- to $41.9+/-12.4 \%$ and \&\#8211;3,45 +/- 5,25 to $47.2+/-12.6 \%$ dilation at maximum flow before and after incubation with 17beta-estradiol in WT $(n=11)$ and beta-ERKO females $(n=12)$ and WT males $(n=13)$, respectively, $\mathrm{p}<0.05$, ANOVA]. Increased dilation to flow was also observed after incubation with PPT in WT females $(9,27+/-5,81$ to $47.7+/-15.7$ before and after incubation with 17 beta-estradiol, $n=7 . P<0.05)$ but not WT males $(-6,99+/-4,58$ to $3.14+/-1.87$, before and after incubation with 17beta-estradiol $n=4$, $P>0.05$ ). Myogenic tone was similar both between groups and between sexes prior to incubation with 17 beta-estradiol $(P>0.05, n=8$ each group, ANOVA). After incubation with 17beta-estradiol a significant decrease in myogeic tone was observed in both WT and beta-ERKO females $(P<0.05, n=11$ and 13 for WT and beta-ERKO, ANOVA) but not in either WT or beta-ERKO males $(P<0.05, n=11$ both groups, ANOVA). Conclusion: The absence of ER-beta is associated with gender specific alteration in flow mediated relaxation in response to 17beta-estradiol. Estrogens, through the action of ER-beta are responsible for estrogen enhance flow mediated dilation in male mice whereas in females ER alpha may mediate this response. Myogenic tone is unaffected by the loss of ER-beta in either male or female mice, however gender specific differences exist in myogenic tone after incubation with 17beta-estradiol. 


\title{
Abtsract \#: $5-3$ \\ RALOXIFENE DILATES RAT PRESSURIZED RESISTANCE-SIZED MESENTERIC ARTERIES: ROLE OF GENDER AND ENDOTHELIAL NITRIC OXIDE
}

\author{
Chan YC; Yao X; Liu CL; Laher I; Vanhoutte PM; Huang Y
}

Department of Physiology, Chinese University of Hong Kong; Department of Pharmacology and Therapeutics, University of British Columbia, Canada; Department of Pharmacology, University of Hong Kong, Hong Kong SAR, China

Estrogen was found to reduce myogenic responses in mammalian resistance arteries. However, modulation by selective estrogen receptor modulators of vessel tone in resistance-sized vessels has not been investigated. The aim of the present study was to determine whether raloxifene, the 2 nd generation selective estrogen receptor modulator could dilate resistance-sized mesenteric arteries isolated from both female and male rats and the possible mechanisms involved in raloxifene-induced vasodilatation. The artery ring segments were cannulated and mounted in a pressure myograph for vessel diameter measurement. The intraluminal pressure was held constant at $80 \mathrm{mmHg}$ throughout the entire duration of the experiment and the maximal dilation was estimated by the end of each experiment using $\mathrm{Ca} 2+$-free and EGTA-containing Krebs solution. In the pressurized vessels, raloxifene caused vasodilatation in a concentration-dependent manner. The threshold concentration is around $0.3 \mathrm{nmol} / \mathrm{L}$ for raloxifene to induce a small dilating effect. Raloxifene displayed higher potency in dilating vessels from female than male rats. Treatment with NG-nitro-L-arginine methyl ester $(30 \mu \mathrm{M})$ slight constricted the vessel but its presence markedly reduced the vasodilator response to raloxifene. In contrast, co-treatment with charybdotoxin and apamin each at $100 \mathrm{nM}$ did not affect raloxifene-induced vasodilatation. In summary, (i) raloxifene potently dilates rat resistance arteries at concentrations equivalent to the therapeutic level of raloxifene used in the treatment of postmenopausal osteoporosis, (iii) endothelial nitric oxide is the major mediator of raloxifene-induced vasodilatation, and (ii) there is a gender difference in vasodilator response to raloxifene. (Supported by a RGC grant) 


\title{
Abtsract \#: $5-4$ \\ SHED MEMBRANE PARTICLES FROM T LYMPHOCYTES IMPAIR ENDOTHELIAL FUNCTION AND REGULATE ENDOTHELIAL PROTEIN EXPRESSION
}

\author{
Tesse A, Martin S, Hugel B, Martínez MC, Morel O, Freyssinet JM, Andriantsitohaina R.
}

Laboratoire de Pharmacologie et Physico-Chimie des Interactions Cellulaires et Moleculaires, CNRS UMR 7034, 74 route du Rhin, B.P. 24, 67401 Illkirch, France.

Introduction: Microparticles (MPs) are membrane vesicles with procoagulant and proinflammatory

properties released from the plasma membrane of various cell types during activation by agonists, shear stress or apoptosis. Background -The present study was designed in dissecting the effects evoked by $T$ lymphocytes-derived MPs on vascular function and in particularly on endothelial cells. Methods: MPs were produced by treatment of the human lymphoid CEM T cell line with actinomycin D or phytohaemagglutin. Mice aortic rings and mesenteric arteries and human umbilical vein endothelial cells were incubated with $30 \mathrm{nmol} / \mathrm{L}$ MPs to test vascular reactivity, protein expression with western blot analysis and calcium measurements. Results: Incubation of mice aortic rings with MPs resulted in a time-dependent impairment of acetylcholine-induced relaxation of pre-contracted vessels, with a maximal reduction after 24 hours. MPs also impaired shear stress-induced dilatation of mice small mesenteric arteries by affecting nitric oxide (NO)- and prostacyclin- but not EDHF-component of the response. However, neither alteration of calcium signaling, in response to agonists, nor reduction of cyclo-oxygenase-1 expression did account for the impairment of $\mathrm{NO}$ and prostacyclin component of the endothelial response. The effect of MPs was rather due to a decrease in expression of endothelial NO-synthase and an overexpression of caveolin-1. Futhermore, lymphocyte-derived MPs from diabetic patients or in vivo circulating MPs from either diabetic or HIV-infected patients reduced endothelial NO-synthase expression. Finally, MPs effects on endothelial cells were not driven through CD11a/CD18 adhesion molecules or Fas/FasL pathway. Discussion: The present study shows that MPs from T cells, at concentration that can be reached in circulating blood in pathological disorders, induce endothelial dysfunction both in conductance and resistance arteries by alteration of $\mathrm{NO}$ and prostacyclin pathways. MPs regulate protein expression for endothelial NO-synthase and caveolin-1. These data contribute to a better understanding of the deleterious effects of enhanced circulating MPs observed in disorders with cardiovascular or immune complications. 


\title{
Abtsrat \#: $5-5$ \\ IMPAIRED MYOGENIC RESPONSE AND DILATION TO ACETYLCHOLINE OF CEREBRAL ARTERIES ISOLATED FROM HYPERCHOLESTEROLEMIC MICE.
}

\author{
Drouin A; Thorin E.
}

Institut de Cardiologie de Montréal, centre de recherche, 5000, rue Bélanger, Montréal, Québec, H1T 1C8, Canada

\begin{abstract}
Introduction. Cerebral arteries develop myogenic tone (MT) in response to a rise in pressure. Although muscular, the endothelium regulates MT. Endothelial integrity is therefore mandatory for an integrated response to pressure. Risk factors such as hypercholesterolemia (HC) that impair endothelial function, favor the incidence of strokes. The impact of $\mathrm{HC}$ on both myogenic and endothelial function in pial vessels is however poorly understood. Purpose. To investigate and compare the myogenic response and the endothelium-dependent dilation of cerebral arteries isolated from 3 month-old wild-type (WT, $n=5)$ and HC (LDLR-/-;hApoB+/+, n=6) mice. Methods. Isolated basilar arteries were mounted in a pressure myograph. In no-flow condition, diameter was recorded with a video dimension analyzer (Living System, Burlington, VT). The pressure - diameter relationship was measured from 20 to $120 \mathrm{~mm} \mathrm{Hg}$. In a second series of experiments, the endothelial function was assessed at $60 \mathrm{~mm} \mathrm{Hg}$. Vessels were constricted to $50 \%$ in both groups with phenylephrine (PE, $0.01 \mathrm{mM})$ or a depolarizing physiological solution $(80 \mathrm{mM}$ $\mathrm{KCl}-\mathrm{PSS}$ ). A concentration-response curve to acetylcholine (Ach) was obtained in the absence or presence of N-nitro-L-arginine (L-NNA, a NOS inhibitor). Data are expressed as mean +/- SEM. A P value lower than 0.05 was considered significant $\left(^{*}\right)$. Results. MT developed in both groups $(n=5)$ at $40 \mathrm{~mm} \mathrm{Hg.} \mathrm{At} 60$ $\mathrm{mm} \mathrm{Hg}, \mathrm{MT}$ represented $15+/-3 \%$ and $12+/-2 \%$ of the maximal diameter in WT and $\mathrm{HC}$ vessels, respectively. In WT arteries, MT was $18+/-2,18+/-2$ and $16+/-2 \%$ at 80,100 and $120 \mathrm{~mm} \mathrm{Hg}$, respectively. In contrast, in $\mathrm{HC}$ vessels, MT fell $\left({ }^{*}\right)$ from $16+/-2$ at 80 , to $11+/-1$ at 100 and $7+/-1 \%$ at $120 \mathrm{~mm} \mathrm{Hg}$. In constricted vessels, Ach induced a dilation prevented $\left(^{*}\right)$ by combining indomethacin, L-NNA and $\mathrm{KCl}$-PSS. In the presence of PE, the potency (pD2) of Ach-induced dilation was similar in WT $(6.4+/-0.5)$ and $\mathrm{HC}(7.2+/-0.1)$ mice, but the efficacy of Ach was reduced $\left({ }^{*}\right)$ from $61+/-8 \%$ in WT, to $38+/-3 \%$ in $\mathrm{HC}$ mice vessels. L-NNA reduced $\left(^{*}\right)$ the maximal dilation to Ach in WT $(26+/-2 \%)$ and HC $(27+/-4 \%)$ vessels. In depolarized conditions, Ach-induced dilation was similar in WT (48+/-5\%) and HC (45+/-6\%) arteries. Conclusion. Our data demonstrate that cerebral arteries from $\mathrm{HC}$ mice do not resist to pressure greater than $80 \mathrm{~mm} \mathrm{Hg}$. In addition, although an endothelial dysfunction has developed, it does not involve NO nor a putative EDHF.
\end{abstract}




\title{
Abtsract \#: 5-6 \\ CONTRIBUTION OF NO PRODUCED BY INDUCIBLE NO SYNTHASE TO VASCULAR RESPONSES OF MESENTERIC ARTERIOLES IN STREPTOZOCIN-DIABETIC RATS
}

\author{
Nakayama K; Inshikawa T; Kohno F; Kawase, R; Yamamoto Y
}

Department of Cellular and Molecular Pharmacology, Graduate School of Pharmaceutical Sciences, University of Shizuoka. 52-Yada, Shizuoka City, Shizuoka 422-8526, Japan

A number of in vitro studies have shown altered arterial contraction and relaxation activities in streptozocin (STZ)-induced diabetic rats. However, these studies have provided conflicting observations as to responses to phenylephrine, an alpha1-agonist, and acetylcholine, an endothelium-dependent vasodilator. Moreover, little information is available about the influence of diabetes on resistance arterioles. The present study was thus undertaken to elucidate whether or not STZ-induced diabetes alters in vivo responses of arterioles. To this end, we used intravital video-microscopy to observe STZ-diabetic rats' mesenteric arterioles with an inner diameter of around 20 micrometer, and investigated the modulatory role of nitric oxide (NO), particularly that derived via inducible NO synthase (iNOS), in both the basal tone and vasoconstrictor response to alpha-adrenergic stimulation. The mesentery was exteriorized from anesthetized rats, spread in a chamber, and superfused with Tyrode solution. Compared with age-matched controls, the diabetic rats showed enhanced vascular sensitivity to phenylephrine contained in the superfusate. The preincubation of the mesentery with Nomega-nitro-L-arginine (L-NNA), a NOS inhibitor, shifted the phenylephrine-concentration-response curves to the left in both the diabetic and control rats. Even in the presence of L-NNA, the sensitivity to phenylephrine was higher in the diabetic rats than in the control. Acetylcholine relaxed the arterioles in both groups, but to a significantly greater extent in the control than in the diabetic rats. However, the L-NNA-induced constriction of arterioles did not differ significantly between the groups. In contrast, the amplitude of the constrictions of the arterioles induced by S-ethylisothiourea, an inhibitor of iNOS, was significantly greater in the microvessels of only the diabetic rats. Immunostaining of the mesentery with a specific antibody for iNOS revealed iNOS in both the smooth muscle layer of the arterioles and in the macrophages in the diabetic mesentery, whereas the control mesenteries showed iNOS only in the macrophages. The present study produced several important findings. First, the constriction responses of the arterioles to phenylephrine were sensitized in the STZ-diabetic rats. The enhanced response to the alpha1 adrenergic agonist does not appear to be attributable to a reduced production of NO in the diabetic rats. Secondary, the arterioles' relaxation responses to acetylcholine were impaired in the STZ-diabetic rats. Finally, regulation of the basal diameter of the arterioles by endogenous NO was altered during diabetes mellitus. iNOS rather than eNOS, appears to profoundly contribute to the regulation of arteriolar diameters in diabetic rats. See for further details: Brit. J. Pharmacol. (2004)141,269-276.doi:10.1038/sj.bjp.0705611 


\title{
Session 6: Reactive oxygen and inflammation
}

\author{
Ahtsract \#: 6-1 \\ IMPAIRED ENDOTHELIUM-DERIVED HYPERPOLARIZING FACTOR IN AGED AND \\ HYPERCHOLESTEROLEMIC MOUSE GRACILIS ARTERY.
}

Krummen S; Thorin E.

Institut de cardiologie de Montréal, Centre de recherche, 5000, rue Bélanger, Montréal, Québec, H1T 1C8, Canada

Introduction. The vascular endothelium releases a dilatory factor that hyperpolarizes the underlying smooth muscle. This endothelium-derived hyperpolarizing factor (EDHF), which is neither nitric oxide (NO) nor prostacyclin, has multiple identities depending both on the species and the tissue studied. Purpose. To characterize the vasodilatory mechanism of EDHF and its response to age and hypercholesterolemia $(\mathrm{HC})$. Methods. Experiments were conducted on pressurized $(80 \mathrm{~mm} \mathrm{Hg})$ isolated gracilis arteries of 3,12 and $20 \mathrm{~m} / \mathrm{o} \mathrm{HC}$ LDL receptor-null mice expressing the human apolipoprotein B-100 (1) using a method previously described (2). Dilation to acetylcholine (Ach, $1 \mathrm{nM}$ to $0.03 \mathrm{mM}$ ) were tested on vessels precontracted with phenylephrine $(0.01 \mathrm{mM})$. In all experiments, L-NNA $(0.1 \mathrm{mM})$ and indomethacin $(0.01$ $\mathrm{mM}$ ) were present in the oxygenated physiological salt solution (PSS; 37oC, $\mathrm{pH} 7.4$ ) to prevent $\mathrm{NO}$ and prostanoid formation. When used, barium $(0.03 \mathrm{mM})$, ouabain $(1 \mathrm{mM})$, apamin (1 microM), charybdotoxin $(100 \mathrm{nM})$ and 17 -octadecynoic acid $(0.01 \mathrm{mM})$ were added to the bath $30 \mathrm{~min}$ before the start of the experiment. Responses to Ach are expressed as \% of the maximal dilation obtained in calcium-free PSS. Results are mean+/-SEM. P less than 0.05 was accepted as significant (Student's t test). Results. In gracilis

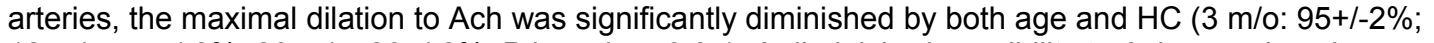
$12 \mathrm{~m} / \mathrm{o}: 55+/-8 \% ; 20 \mathrm{~m} / \mathrm{o:} 26+/-2 \%$; $P$ less than 0.05). A diminished sensibility to Ach was also observed in 12 and $20 \mathrm{~m} / \mathrm{o}$ arteries (pD2: 6.3+/-0.1) versus $3 \mathrm{~m} / \mathrm{o}$ arteries(pD2: 7.4+/-0.1) (P less than 0.05). In all groups, combination of apamin and charybdotoxin reduced EDHF dilation (3 m/o: $21+/-4 \%$; $12 \mathrm{~m} / \mathrm{o}$ : $7+/-2 \% ; 20 \mathrm{~m} / \mathrm{o}: 2+/-1 \%$ ) (P less than 0.05 ). While ouabain alone blocked EDHF dilation in $20 \mathrm{~m} / \mathrm{o}$ arteries $(2+/-1 \%)$, only its combination with barium and 17-octadecynoic acid completely blocked EDHF dilation in both 3 and $12 \mathrm{~m} / \mathrm{o}$ arteries (5+/-3\% and $11+/-4 \%$ respectively). Conclusion. Age and HC impair EDHF-mediated dilation in the gracilis mouse artery. In all three groups, Ach stimulates SKCa and IKCa channels thus increasing $\mathrm{Na} / \mathrm{K}$ ATPase pump activity, which solely account for EDHF-dependent dilation in $20 \mathrm{~m} / \mathrm{o} \mathrm{HC}$ mice. In 3 and $12 \mathrm{~m} / \mathrm{o} \mathrm{HC}$ mice, Na/K ATPase pump, Kir channels and a cytochrome P450 metabolite contribute to EDHF-mediated dilation. (1) Sanan et al. (1998). Proc Natl Acad Sci USA, 95, 4544-4549; (2) Nguyen et al. (1999). Cardiovasc Res, 43, 755-761. 


\title{
Ahtsract \#: 6-2 NITRIC OXIDE FROM INOS AND SUPEROXIDE ANION MEDIATE THE IMPAIRMENT OF
BRADYKININ-RELAXATION AFTER LPS IN RAT CEREBRAL ARTERIES
}

\author{
Hernanz R.(1); 1Briones A.M.; 1,4Alonso M.J.; 2Vila E.; 3Simonsen U.; 1García A.; 4Pérez V. and
} 1Salaices $M$.

1Departamento de Farmacología y Terapéutica, Facultad de Medicina, Universidad Autónoma de Madrid, 28029 Madrid, Spain, 2Departament de Farmacología Terapéutica i Toxicología, Facultat de Medicina, Universitat Autònoma de Barcelona, 08193 Bellaterra, Barc

Introduction: Despite systemic hypotension, endotoxic shock is associated with an early decrease in cerebral blood flow and perfusion pressure as well as increased cerebrovascular resistance. These cerebrovascular alterations would be involved in the impaired neurological function found in sepsis. Background and purposes: This study was performed to analyze the effect of lipopolysaccharide (LPS) on bradykinin (BK)-induced relaxation in rat middle cerebral arteries and the role of inducible nitric oxide synthase (iNOS) and cyclooxygenase-2 (COX-2)-derived metabolites and reactive oxygen species in this effect. Material and methods: Tension was measured in wire myographs, protein expression was evaluated by Western blot and immunohistochemistry, and superoxide anion and TXA2 measurements were analyzed by hydroethidium staining and enzyme immunoassay, respectively. Results: LPS (1-5 h, $10 \& \# 61472 ; \& \# 61549 ; \mathrm{g} / \mathrm{ml})$ reduced the relaxation induced by BK $(1 \mathrm{nM}-10 \mu \mathrm{M})$ and the NO donor DEA-NO $(0.1 \mathrm{nM}-10 \mu \mathrm{M})$. However, LPS had not effect on the relaxation induced by papaverine $(0.1 \mathrm{nM}-0.1 \mathrm{mM})$, the BK-relaxation after NO synthesis inhibition with L-NMMA and the expression of endothelial NOS, suggesting the specificity of LPS effect on NO relaxation. The respective iNOS and COX-2 inhibitors, aminoguanidine (0.1 mM) and NS 398 (10 \&\#61549;M), and the superoxide anion scavenger, SOD $(100 \mathrm{U} / \mathrm{ml})$, reduced the LPS effect on BK-relaxation; however, the TXA2/PGH2 receptor antagonist, SQ 29,548 (1 \&\#61549;M), and the hydrogen peroxide scavenger, catalase (1000 U/ml), did not modify the LPS effect. COX-2 expression was increased at 1 and $5 \mathrm{~h}$ of LPS incubation, while that of iNOS, Cu/Zn-SOD and Mn-SOD was only increased after $5 \mathrm{~h}$. All three vascular layers expressed COX-2 and $\mathrm{Cu} / \mathrm{Zn}-\mathrm{SOD}$. iNOS expression was detected in the endothelium and adventitia after LPS. LPS ( $5 \mathrm{~h}$ ) increased superoxide anion but not TXA2 levels. Discussion: LPS inhibited the BK-relaxation by a mechanism that reduced the NO-mediated relaxation. Superoxide anion, which could be generated in part from COX-2, and NO from iNOS participate in the LPS effect. This effect would contribute to explain the decrease in cerebral blood flow and perfusion pressure and the increase in cerebrovascular resistance observed in the earlier stages of endotoxic shock. (Supported by DGICYT, FISS and the EC VASCAN 2000 Consortium) 


\title{
Abtsract \#: $6-3$ \\ ROLE OF TOLL-LIKE RECEPTOR 2 AND TOLL-LIKE RECEPTOR 4 IN POST-ISCHEMIC CORONARY ENDOTHELIAL DYSFUNCTION IN MICE
}

\author{
Favre J; Musette P; Henry JP; Thuillez C; Richard V
}

U644 U519 IFR23, Rouen university medical school 22 bd Gambetta Rouen, 76183, France

Introduction: A growing body of evidence suggests a role of the toll-like receptors (TLR) in inflammatory processes. In addition to LPS, TLR are activated by many endogenous ligands such as heat shock proteins and oxygen-derived free radicals which are both produced during cardiac ischemia-reperfusion (I/R). Among TLR, TLR-2 and TLR-4 are expressed in endothelial and myocardial cells and appear to regulate neutrophil-endothelial interactions. Since neutrophil adhesion is a critical event in endothelial injury induced by I/R, we assessed whether TLR-2 and TLR-4 were involved in I/R induced coronary endothelial injury. Material and methods: Wild type (WT) and TLR-2 or TLR-4 knock-out (TLR-2 KO, TLR-4 KO) mice were subjected to sham surgery or 30 minutes of left anterior descending coronary ligation followed by 60 minutes of reperfusion. Coronary segments (diameter, 170 to $200 \mu \mathrm{m}$ ) were then removed distal to the site of occlusion and mounted in a wire myograph. In parallel, neutrophil accumulation was assessed by measuring myeloperoxidase (MPO) activity in tissue previously subjected to I/R. Results: I/R reduced the distal relaxation to acetylcholine in WT mice (maximal relaxation: sham WT 68+/-5\%; I/R WT 34+/-4\% $\mathrm{p}=0.006$ ). In contrast, this reduced response to acetylcholine was absent in TLR-4 KO mice (sham KO4 $62+/-6 \%$; I/R KO4 65+/-6\%) as well as in TLR-2 KO mice, in which I/R paradoxically improved the relaxation (sham KO2 63+/-9\%; I/R KO2 81+/-12\% p=0.001 vs I/R WT). The endothelium-independent response to the NO donor sodium nitroprusside was not affected in all groups. Moreover, the increased MPO activity observed after I/R (U/100mg tissue: sham WT 53+/-17; I/R WT 133+/-12 p=0.003) was significantly reduced in TLR-4 KO mice (I/R KO4 103+/-9 p=0.023 vs I/R WT) and absent in TLR-2 KO mice (I/R WT 133+/-12; I/R KO2 51+/-15 p=0.004) showing a decrease in neutrophil infiltration.

Discussion: This study is, to the best of our knowledge, the first to report post-ischemic coronary endothelial injury in mice. We also demonstrate for the first time that TLR-2 and TLR-4 signaling contribute importantly to the endothelial dysfunction that occurs following $\mathrm{I} / \mathrm{R}$, possibly by reducing neutrophil adhesion and thus neutrophil-mediated endothelial injury. 


\title{
Abtsract \#: 6-4 \\ POTENTIAL ROLE OF SORBITOL PATHWAY IN THE DEVELOPMENT OF OXIDATIVE STRESS-INDUCED DYSFUNCTION OF SKELETAL MUSCLE ARTERIOLES IN DIABETES MELLITUS
}

\author{
Tóth E, Bagi Z, Jolayemi K, Koller A
}

Department of Pathophysiology, Semmelweis University, 1445 Budapest, Hungary Department of Physiology, New York Medical College, Valhalla NY 10595 USA

\begin{abstract}
In diabetes mellitus high plasma glucose levels contribute to the development of endothelial dysfunction, likely via an increased generation of reactive oxygen species. The exact mechanism, however, has not yet been fully elucidated. Sorbitol synthesis via the polyol pathway represents a minor $(<3 \%)$ fate of glucose at physiological concentrations, however, in diabetes mellitus via aldose reductase $\sim 35 \%$ of glucose could be converted to sorbitol. We hypothesized that higher concentrations of sorbitol, due to increased oxidative stress, elicit endothelial dysfunction in microvessels. Thus, changes in diameter of isolated, pressurized rat skeletal muscle arterioles (diameter: 140 \&\#956;m) in response to increasing concentrations of sorbitol (10-9-10-4 M) were investigated. Sorbitol, administered extraluminally, elicited dose dependent constrictions (max.: $24 \pm 5 \%$ ), which were partly inhibited by either superoxide dismutase (SOD, $120 \mathrm{U} / \mathrm{mL}$ ) or catalase $($ CAT, $80 \mathrm{U} / \mathrm{mL}$ ), whereas simultaneous administration of SOD and CAT abolished completely the sorbitol-induced constrictions. Furthermore, incubation of arterioles with sorbitol (0.01 \&\#956;M, intraluminally for $40 \mathrm{~min}$ ) significantly decreased acetylcholine-induced dilations (control max. dil: $95 \pm 7 \%$ vs. $14 \pm 5 \%$ ), and converted flow-induced dilations to constrictions (control max. dil: $36 \pm$ $1 \%$; max cons.: $20 \pm 2 \%$ ), but it did not affect dilations to adenosine (control max. dil.: $36 \pm 2 \%$ vs. $30 \pm 4 \%$ ). Thus, we suggest that higher intracellular levels of sorbitol, via oxidative stress, promote endothelial dysfunction, which could lead to increases in arteriolar resistance and disturbances in the regulation of skeletal muscle blood flow in diabetes mellitus. (Supported by Hungarian NSRF OTKA, T-034779, and the NHLBI, HL-46813 USA)
\end{abstract}




\title{
Abtsract \#: 6-5 \\ ROS-DEPENDENT MODULATION OF MYOGENIC TONE IN CRITICAL LIMB ISCHAEMIA
}

\author{
Coats P; Wadsworth RM.
}

University of Strathclyde, Dept Physiology and Pharmacology, 27 Taylor Street Glasgow G4 ONR, UK

Introduction: Critical limb ischaemia (CLI) is associated with an inability to regulate blood volume within the capillary beds of the diseased part of the leg. Part of the sequelae accompanying CLI can be associated with dysfunction of resistance arterioles. Background and purposes: Reactive oxygen species (ROS) were originally considered toxic metabolites. Increasing evidence suggests that they can play an important signalling role in essential physiological functions including pressure-dependent arteriolar myogenic tone1. In addition to the well documented calcium/ calmodulin/ myosin light chain kinase dependency of myogenic contraction evidence suggests that $G$ and $F$ actin based structures contribute to myogenic tone2. In this present study we examined ROS and G/F actin mechanisms of arteriolar contractile responses in skeletal muscle arterioles isolated from the diseased part of the leg (DSM) vs. arterioles isolated form the healthy part of the leg (PSM, internal control). Materials and methods: Resistance arterioles (lumen diameter $\sim 80 \mathrm{uM}$ ) were isolated from skeletal muscle biopsies taken from subjects with CLI. Pressure-dependent and pressure-independent mechanisms of vascular tone were studied (Danish MyoTech P110 pressure myograph). Results and discussion: There was no difference in pressure-independent mechanisms of vascular tone (DSM vs. PSM). Pressure-dependent myogenic constriction was reduced $(20.9+/-2.3 \%$ vs. $4.2+/-1.2 \% \& 28.2+/-3.1 \%$ vs. $6.8+/-1.4 \%$, PSM vs. DSM at $80 \mathrm{mmHg} \& 120 \mathrm{mmHg}$ respectively; $n=6$ pairs). The antioxidant NAC, the inhibitor of $\mathrm{NAD}(\mathrm{P}) \mathrm{H}$ oxidase $\mathrm{DPI}$ and cytochalasin $\mathrm{D}$, an inhibitor of actin polymerisation inhibited myogenic responses in PSM arterioles. NAC, DPI and cytochalasin D had no effect on pressure-dependent myogenic contraction measured in the DSM arterioles. NAC, DPI and cytochalasin D actions were selective to myogenic contraction as they had no effect on pressure-independent contraction. Based on these results we propose that the myogenic response involves two discrete contractile pathways. One pathway involves the conventional signalling pathway for vascular smooth muscle constriction. The other involves activation of $\mathrm{NAD}(\mathrm{P}) \mathrm{H}$ oxidase, elevation of ROS and actin polymerization. Since the NAD $(P) H / R O S /$ actin polymerization-dependent response is absent in arterioles associated with $\mathrm{CLI}$, we propose that deregulation of this second pathway negates the ability of these arterioles to resist pressure-dependent forced dilatation and generate a myogenic contraction and this dysfunction significantly contributes to the altered vascular function associated with CLI. 1. Nowicki et al. Circ Res. 2001;89:114-6 2. Gokina NI, Osol G. Am J Physiol. 2002;282:H1410-20. 


\title{
Abtsract \#: 6-6 \\ UPREGULATION OF PRO-INFLAMMATORY PROTEINS THROUGH NF-א;B PATHWAY BY SHED MEMBRANE MICROPARTICLES RESULTS IN VASCULAR HYPOREACTIVITY
}

\author{
Tesse A, Hugel B, Martínez MC, Chalupsky K, Muller CD, Mitolo-Chieppa D, Freyssinet JM,
} Andriantsitohaina R.

Laboratoire de Pharmacologie et Physico-Chimie des Interactions Cellulaires et Moleculaires, CNRS UMR 7034, 74 route du Rhin, B.P. 24, 67401 Illkirch, France.

INTRODUCTION: Recent data suggest that inflammation play a central role in the origin and complications of cardiovascular disease. The mechanisms triggering the modifications of the vessel contraction/relaxation balance in inflammatory diseases are not fully elucidated. Microparticles are membrane vesicles with procoagulant and proinflammatory properties released during cell activation, including apoptosis. Several lines of data support the hypothesis that MPs contribute to the pathogenesis of cardiovascular diseases, however, evidences for a direct role of MPs and the mechanisms by which they could impair the regulation of vascular reactivity are still limited. BACKGROUND: The present study was designed in dissecting the effects evoked by microparticles on vascular reactivity. METHODS: Mice aortic rings were incubated with MPs from either apoptotic $T$ lymphocytes or from diabetic patients and mounted on a wire myograph. Concentration-response curves were constructed by cumulative application of either serotonin or phenylephrine in vessels with or without functional endothelium. The vessels were used for Western blot analysis and immunohistochemical labeling for assessment of inducible NO-synthase (iNOS) and cyclooxygenase-2 (COX-2) expression and localization. RESULTS: Microparticles from either apoptotic $T$ lymphocytic cells or diabetic patients induced vascular hyporeactivity in response to vasoconstrictor agents in mouse aorta. Hyporeactivity was reversed by nitric oxide (NO)-synthase plus cyclooxygenase- 2 inhibitors, and associated with an increased production of vasodilatory products such as $\mathrm{NO}$ and prostacyclin. Microparticles induced an up-regulation of pro-inflammatory protein expressions, iNOS and COX-2 through NF-к;B-dependent transcription via Fas/FasL pathway. DISCUSSION: Several pathologies are associated with vascular dysfunction including attenuation of endothelium-dependent vasodilatation, alteration of responsiveness of vascular smooth muscle to vasoconstrictor stimuli or both. These data provide a rationale to explain the paracrine role of microparticles as vectors of transcellular exchange of message in promoting vascular dysfunction during inflammatory disease. 


\section{Abtsract \#: 6-7 \\ UPREGULATION OF NITROTYROSINE, A MARKER FOR PEROXYNITRITE, IN PULMONARY ARTERIES OF PULMONARY HYPERTENSIVE PATIENTS}

Wadsworth RM (1); Hoehn T (2); McPhaden AR (3), Stiller B (4) and Preston AA (1)

(1) University of Strathclyde, Glasgow, Scotland ; (2) Heinrich Heine University, Duesseldorf, Germany Royal Infirmary, Glasgow, Scotland and (4) German Heart Institute, Berlin, Germany

Introduction: Superoxide and peroxynitrite can damage the pulmonary endothelium and could contribute to the progression of pulmonary hypertension. We hypothesised that NO synthase would be upregulated and peroxynitrite availability would be enhanced in pulmonary artery early in the onset of pulmonary hypertension. Materials/Methods: Postmortem lung tissue was wax embedded, sectioned and stained for eNOS, iNOS, nNOS and nitrotyrosine (which records a footprint of peroxynitrite exposure) by immunocytochemistry, and the staining intensity graded. We studied lung tissue from infants who had died from persistent pulmonary hypertension of the newborn $(n=4)$ or from congenital heart defects $(n=26)$, compared to age-matched control patients who had no pulmonary or cardiovascular illness. Results: The cardiac patients had increased staining for eNOS, iNOS and nitrotyrosine in the endothelium of small pulmonary arteries. The staining intensity (cases, controls) was eNOS $1.04+/-0.11,0+/-0$; iNOS $0.60+/$ $0.10,0.13+/-0.08$; nitrotyrosine $1.00+/-0.11,0.25+/-0.09$ units (all $\mathrm{P}<0.01$ ). Individual patients who had high levels of eNOS, were also found to have high levels of iNOS and of nitrotyrosine. There was no upregulation of nNOS in the patients. Similar results were found in the patients with persistent pulmonary hypertension of the newborn. Discussion: The upregulation of eNOS and iNOS appears to be linked to pulmonary hypertension, since it was found in the pulmonary artery endothelium, but not in other locations within the lung where eNOS and iNOS occur. The rise in nitrotyrosine suggests that superoxide availability may be increased in pulmonary hypertension. The correlation of eNOS with iNOS and nitrotyrosine suggests a linkage between these pathways in the adaptation of the pulmonary vascular endothelium to pulmonary hypertension. 


\title{
Abtsrat \#: $6-8$ \\ OXIDATIVE STRESS INDUCES IRREVERSIBLE DAMAGE TO THE RENAL ARTERY ENDOTHELIAL FUNCTION BEYOND 12 MONTHS OF AGE IN HYPERCHOLESTEROLEMIC MICE
}

\author{
Gendron M-E; Thorin E
}

Institut de Cardiologie de Montréal, Centre de recherche, 5000, rue Bélanger, Montréal, Québec, H1T 1C8, Canada

Introduction. Endothelial dysfunction is an early marker of vascular diseases. Endothelium-dependent dilation has been shown to be impaired both in hypercholesterolemic patients and in animal models. The pathogenic contribution of oxidized LDL to the endothelial dysfunction has been recognized early and suggested that oxidative stress was an important catalyst in the processes leading to vascular diseases. The temporal evolution of the endothelial dysfunction, from its genesis to its irreversible damage however, has not been studied. Purpose. To assess the temporal evolution of the renal endothelial function in a mouse model of hypercholesterolemia. Methods. Experiments were conducted in pressurized $(100 \mathrm{~mm} \mathrm{Hg})$ isolated renal arteries of young $(3 \mathrm{~m} / \mathrm{o})$, mature $(6 \mathrm{~m} / \mathrm{o})$, adult $(12-14 \mathrm{~m} / \mathrm{o})$ and old $(20 \mathrm{~m} / \mathrm{o})$ hypercholesterolemic LDL receptor-null mice expressing the human apolipoprotein B-100. Dilation to acetylcholine (Ach, $1 \mathrm{nM}$ to $0.03 \mathrm{mM}$ ) was tested on vessels contracted with phenylephrine $(0.03 \mathrm{mM})$. To assess the acute contribution of reactive oxygen species on the endothelium-dependent dilation induced by Ach, vessels were treated with the antioxidant N-acetyl-L-cystein (NAC, $0.01 \mathrm{mM}) 30$ min before the cumulative addition of Ach. Data are mean +/- SEM. A P value lower than $0.05\left(^{*}\right)$ was considered statistically significant (ANOVA, Scheffé's F test). Results. Phenylephrine-induced contraction was not different in arteries isolated from $3(76+/-2 \%$ reduction in maximal diameter), $6(70+/-5), 12(69+/-2)$ and $20(53+/-8) \mathrm{m} / \mathrm{o}$ mice. ACh induced a complete endothelium-dependent dilation of renal arteries isolated from $3 \mathrm{~m} / \mathrm{o}$ mice (93+/-4\%). This response decreased $\left({ }^{*}\right)$ at $6 \mathrm{~m} / \mathrm{o}(61+/-10 \%), 12 \mathrm{~m} / \mathrm{o}(64+/-10)$ and $20 \mathrm{~m} / \mathrm{o}$ $(34+/-13)$. Vascular sensitivity to Ach (pD2 $6.4+/-0.2$ at $3 \mathrm{~m} / \mathrm{o})$ only decreased significantly ${ }^{*}$ ) at 12 $(5.7+/-0.2)$ and $20 \mathrm{~m} / \mathrm{o}(5.7+/-0.3)$. Acute exposure of the vessels to NAC did not affect Ach-induced dilation in $3 \mathrm{~m} / \mathrm{o}$ mice. NAC restored $\left({ }^{*}\right)$ Ach-induced dilation in arteries isolated from $6 \mathrm{~m} / \mathrm{o} \mathrm{mice}$ $(88+/-4 \%)$ but prevent neither the reduced dilation to Ach in 12 and $20 \mathrm{~m} / \mathrm{o}$, nor the lower vascular sensitivity to Ach. Conclusion. Hypercholesterolemia is associated with the development of an early endothelial dysfunction. This is associated with a rise in oxidative stress, which generates irreversible damage beyond 12 months of age. 
Abtsract \#: 6-9

AXON REFLEX-RELATED CUTANEOUS VASODILATATION TO ANODAL CURRENT APPLICATION IN HUMAN IS NOT IMPAIRED BY CYCLOOXYGENASE-2 BLOCKADE.

Tartas M; Durand S; Koïtka A; Bouyé P; Saumet JL; Abraham P

CNRS UMR 6188 Circulations Régionales et Microcirculation, University of Medicine, Rue Haute de Reculée, 49045 Angers cedex 01 France

Introduction: It is generally acknowledged that the cutaneous vasodilatation to monopolar galvanic current application would result from an axon reflex in primary afferent fibres and to the neurogenic inflammation resulting from neuropeptide release. Evidence has been provided for a participation of prostaglandin (PG) in anodal current-induced cutaneous vasodilatation. Thus, the inducible cyclooxygenase isoform (COX-2), assumed to play a key role in inflammation, should be involved in the synthesis of the PG released. Material and method: Skin blood flow (SkBF) variations induced by 5-min, 0.1-mA monopolar anodal current application was evaluated with laser-Doppler flowmetry on the forearm in 8 healthy volunteers under indomethacin (COX-1 and 2 inhibitor), celecoxib (COX-2 inhibitor) or placebo treatment. SkBF was indexed as cutaneous vascular conductance (CVC) expressed as percent of heat-induced maximal CVC (\%MVC). Results: No difference was found in CVC values at rest: $14.3+/-4.0$, $11.9+/-3.2,10.9+/-2.0 \%$ MVC after indomethacin, celecoxib or placebo treatment, respectively. At 10-min following the onset of anodal current application, CVC values were $22.2+/-4.9 \% \mathrm{MVC}$, (non-significant from rest) under indomethacin, 85.7 +/- 15.3\%MVC $(p<0.001$ from rest) under celecoxib and $70.4+/-13.1 \% M V C$ ( $p<0.001$ from rest) under placebo. Discussion: Indomethacin but not celecoxib treatment significantly inhibited the vascular response to anodal current application. Thus, although assumed to result from an axon reflex in primary afferent fibres and neurogenic inflammation, these results suggest that the anodal current-induced vasodilatation is mainly dependent on COX-1 prostaglandin synthesis. 


\title{
Abtsrat \#: 6-10 \\ SULFAPHENAZOLE REDUCES POST-ISCHEMIC VASCULAR DYSFUNCTION
}

\author{
Bai N;Hunter AL;Granville DJ and Laher I
}

Dept Pharmacology \& Therapeutics and iCAPTURE Centre for Cardiovascular and Pulmonary Research, University of British Columbia, Vancouver, BC, Canada

We recently demonstrated that cytochrome p450 2C (CYP2C) inhibitors significantly reduce infarct size caused by cardiac ischemia and reperfusion (I/R) using a rat Langendorff perfusion model of global ischemia and a rabbit LAD occlusion model of regional ischemia (Proc Natl Acad Sci USA.

2004;101:1321-6). CYP2C inhibition reduced superoxide generation and augmented coronary flow. In this study, we hypothesize that CYP2C-mediated superoxide production results in nitric oxide scavenging, production of peroxynitrite and reduced endothelial-dependent, nitric oxide-mediated vasodilation. To test this hypothesis, rat hearts were perfused in Langendorff mode for 20 min in the presence, or absence, of the specific CYP2C9 inhibitor sulfaphenazole (10 (M) and then subjected to 30 min global no-flow ischemia followed by $15 \mathrm{~min}$ reperfusion. Septal coronary resistance arteries were isolated and mounted on glass cannulae for measurements of luminal diameter. Arterial tone was increased with U-46619 and tissues were exposed to increasing concentrations of acetylcholine (1 nM-10 (M) to elicit endothelial-dependent, nitric oxide-mediated vasodilation. Acetylcholine caused near maximal dilation in control tissues not subjected to I/R. Following $30 \mathrm{~min}$ global ischemia and $15 \mathrm{~min}$ reperfusion, endothelial-dependent vasodilation occurred with reduced sensitivity and the maximal effect of acetylcholine was also attenuated. Pretreatment with sulfaphenazole restored both the sensitivity and maximal effect of acetylcholine. In summary, sulfaphenazole restored post-ischemic endothelium-dependent, nitric oxide-mediated vasodilation. This work was supported by grants from the CIHR (DG) and HSFC (IL) 
Abtsrat \#: 6-11

IL-1ß IMPAIRS NITRIC OXIDE MEDIATED RELAXATION IN RAT MESENTERIC RESISTANCE ARTERIES

Jiménez-Altayó F, Briones AM, Giraldo J and Vila E.

Dpt. Farmacologia,Terapèutica i Toxicologia, Facultat de Medicina, Universitat Autònoma de Barcelona. Bellaterra 08193, Barcelona.

Introduction: Inflammatory cytokines like IL-1ß are associated to altered vascular responses in different vascular beds. Background and purposes: We and others have previously described the involvement of several mediators in the impairment by cytokines or bacterial lipolysaccharide of endothelium dependent relaxations in several vascular beds. The aim of the present study was to analyse the mechanisms involved in the endothelium dependent relaxations by Interleukin-1ß (IL-1ß) in rat mesenteric resistance arteries. Material and Methods: Second and third branches of mesenteric artery from 3 months-old male Sprague-Dawley rats were incubated in culture medium in either the absence or the presence of IL-1ß\&\#61472; (10 ng/ml, $14 \mathrm{~h})$. Parallel experiments were performed in arteries freshly isolated from the vascular bed. For diameter measurements $3 \mathrm{rd}$ branches of mesenteric arteries were placed in a pressure myograph and concentration response curves to agonists were performed in the absence and in the presence of different inhibitors. 2nd and 3rd branches of mesenteric artery were used to determine protein expression by Western Blot. Hydroethidine was used to detect superoxide anion (O2.-) in the vessel wall. Results: Acetylcholine (ACh) and sodium nitroprussiate (SNP) relaxed phenylephrine precontracted arteries in a concentration related manner. The concentration response curve to both agonists were shifted to the right by culture medium. The presence of IL-1ß almost abolished the relaxations induced by ACh and SNP. However, IL-1\&\#61538; did not affect the relaxation induced by either 8-bromoguanosine 3',5'-cyclic monophosphate or papaverine. Soluble guanylil cyclase and eNOS protein expression were similar in all experimental conditions studied. Superoxide dismutase (SOD) improved the impared ACh induced relaxation in the absence but not in the presence of IL-1ß. Nevertheless, the SOD permeable analogue polyethylene glycol-SOD (PEG-SOD) significantly improved the impairment by IL-1ß of ACh vasodilatation. These results correlate with an increase on $\mathrm{O} 2$.- production found in the three layers of the vascular wall after IL-1ß incubation. The effect of IL-1ß on ACh and SNP relaxation was reduced by the xanthine oxydase inhibitor allopurinol. Both PEG-SOD and allopurinol abolished the increase on O2.production. Discussion: These results suggests that the impairment of NO mediated relaxation induced by $\mathrm{IL}-1 ß$ is mediated, at least in part, by an excess of O2.- production likely altering the bioavailability of NO. (Supported by DGCYT (2003-01001) and Fundación Salud 2000) 
Abtsract \#: $6-12$

COX-2 AND INOS IN OMENTAL ARTERIES HARVESTED FROM PATIENTS WITH SEVERE LIVER DISEASES: IMMUNO-LOCALIZATION AND INFLUENCE ON VASCULAR TONE.

Tabernero A, Schneider F, Potenza MA, Fidi-Soa Randriamboavonjy V, Chasserot S, Wolf P, Mitolo-Chieppa D, Stoclet JC, Andriantsitohaina R.

Laboratoire de Pharmacologie et Physicochimie des Interactions Cellulaires et Moleculaires, CNRS (UMR 7034), Illkirch, France.

INTRODUCTION. High cardiac output and low peripheral resistance are the hemodynamic features observed in cirrhosis and acutely developing end-stage liver diseases. The biological mechanisms involved in this low peripheral resistance in patients are far from clear. BACKGROUND: The aim of the present study were to learn whether acutely developing liver diseases and cirrhosis are similar as far as the immuno-location of both COX-2 and iNOS in the vascular wall is concerned, and whether such an enzyme up-regulation has similar consequences on vascular tone. At stake is a better knowledge of the vascular consequences. METHODS. The expression of inducible cyclooxygenase (COX-2) and inducible nitric oxide synthase (iNOS) and the role of vasodilatory prostanoids and endogenous nitric oxide (NO) was investigated in small omental arteries harvested from patients with severe liver diseases, realizing ex vivo study of resistance arteries. Patients were divided into three groups according to the cause of their liver injury: twenty patients undergoing liver transplantation for fulminant hepatic failure (FHF, $n=6)$,

cirrhogenous viral hepatitis $(\mathrm{CH}, \mathrm{n}=6)$ and limited hepatocarcinoma (controls, $n=8)$. The arteries were used for Western blot analysis and immunohistochemical labeling for assessment of COX-2 and iNOS expression and localization and ex vivo vascular reactivity studies. RESULTS: Significant up-regulation of COX-2 and iNOS expressions were detected in arteries from FHF and $\mathrm{CH}$ patients with a greater increase in the former than in the latter. Ex vivo contractile responses to norepinephrine and the thromboxane $\mathrm{A}(2)$ analog, U46619, were not significantly different between patients with severe liver dysfunction and controls. Exposure to either the NO-synthase inhibitor, N(G)-nitro-L-arginine methyl ester (L-NAME), the cyclooxygenase inhibitor, indomethacin, or their combination did not significantly modify contractions of agonists in controls and $\mathrm{CH}$ patients. In FHF, the specific COX-2 inhibitor, $\mathrm{N}$-(2-cyclohexyloxy-4-nitrophenyl) methanesulfonamide (1 micro $\mathrm{m} / \mathrm{l})$, but not L-NAME, significantly enhanced the maximal effect and the sensitivity to norepinephrine. DISCUSSION: This study provide evidence for COX-2 and iNOS upregulation in omental arteries from patients with cirrhogenous hepatitis and fulminant hepatic failure. Whereas neither NO nor vasodilatory prostaglandins seem to play a major role in counteracting arterial contractility of arteries from control patients, COX-2 derivatives are involved in lowering the arterial contractility of vessels harvested from FHF patients. 


\section{Abtsrat \#: 6-13 \\ CYCLOOXYGENASE-2 EXPRESSION AND ROLE OF VASOCONSTRICTOR PROSTANOIDS IN SMALL MESENTERIC ARTERIES FROM PATIENTS WITH CROHN'S DISEASE.}

Tabernero A, Reimund JM, Chasserot S, Muller CD, Andriantsitohaina R.

Pharmacologie et Physico-Chimie des Interactions Cellulaires et Moleculaires, Illkirch, France.

INTRODUCTION: Among the proposed hypothesis for Crohn's disease (CD) pathogenesis, a vascular cause has been suggested, mostly in reference to anatomic and pathological studies. BACKGROUND: The present study investigates the vascular reactivity and the involvement of nitric oxide and prostanoids in regulating vasoconstriction of small mesenteric arteries from patients with $C D$ to understand the vascular component of this pathology. METHODS: Fragments of inflamed intestinal mucosa in CD patients and normal mucosa in controls were used for cytokine immunoassays and histological inflammation scoring. Small mesenteric arteries were tested in contraction experiments and COX-2 and iNOS immunostaining was performed in arterial segments. RESULTS: An increased production of proinflammatory cytokines (tumor necrosis factor-alpha and interleukins 1 beta, 6, and 8) has been observed in biopsy specimens of inflammatory intestinal mucosa. However, contractile responses of small mesenteric arteries from CD patients in response to norepinephrine were not changed ex vivo when compared with controls. Exposure to either the nitric oxide synthase inhibitor $N(G)$-nitro-L-arginine or the cyclooxygenase (COX) inhibitor indomethacin did not modify contractions induced by norepinephrine in either control or CD patients. However, in the latter, the specific COX-2 inhibitor N-(2-cyclohexyloxy-4-nitrophenyl) methanesulfonamide significantly attenuated norepinephrine-induced vasoconstriction. Furthermore, immunohistochemical analysis showed marked COX-2 expression in the whole arterial wall of vessels from CD patients. Vessels from control patients exhibited weak COX-2 staining in the adventitial and endothelial layers only.

DISCUSSION: The above results provide direct evidence for COX-2 expression in small mesenteric arteries from CD patients. They also shed new light on the involvement of vasoconstrictor metabolites of COX in regulating contraction of these arteries. Of particular interest is the balance between vasoconstrictor products from COX-2 and unidentified vasodilatory products that maintained vascular reactivity in a physiological range despite an increase of circulatory cytokines in patients with CD. 


\title{
Session 7: Hypertension
}

\author{
Ahtsract \#: $7-1$ \\ ABNORMAL CONTROL OF PERIPHERAL ARTERIAL RESISTANCES IN THE AGED SPONTANEOUSLY \\ HYPERTENSIVE RAT INVESTIGATED BY IN VIVO NMR IMAGING OF PERFUSION
}

\author{
Bertoldi B, Parzy E, Fromès Y, Leroy-Willig A, Wary, C, Carlier PG
}

NMR Laboratory, AFM-CEA, Institute of Myology, IFR14, Babinski bldg, Pitié-Salpêtrière University Hospital, 83, Bd de l'Hôpital, 75653 Paris cedex 13, France

\begin{abstract}
Increased arterial peripheral resistance and decreased maximum vasodilation have long been identified as essential features of chronic hypertension. By contrast, there is little data on possible alterations in temporal patterns of the vasodilatory responses to stimuli in hypertension. This question was addressed by using NMR imaging combined to arterial spin labeling (ASL), a non-invasive and quantitative technique to evaluate tissue perfusion, with high temporal $(\sim 10 \mathrm{~s})$ and spatial $(\sim 1 \mathrm{~mm} \times 1 \mathrm{~mm})$ resolution. ASL makes use of the arterial water spins as diffusible natural markers and is emerging as the method of choice for in-vivo non-invasive determination of perfusion in most static organs. Ninety-week old male SHR $(n=7)$ and WKY $(n=8)$ rats were studied under pentobarbital anesthesia. Blood pressure was monitored via a carotid catheter throughout the protocol and an air-cuff was wrapped around the thigh to control leg perfusion. The animal was laid inside a dedicated NMR transmitter probe centered in a 4T magnet, with the calf positioned on a surface receiver coil. Calf muscle perfusion was measured non-invasively using ASL sequences, at rest and during reactive hyperemia following total ischemias of either 5 or 30 min duration. Reactive hyperemia profiles differed depending on the duration of the ischemia. Typically, in the WKY rats, a 5-min ischemia induced a brief peak of hyperemia lasting no more than 63 s while 30 min of ischemia was followed by a prolonged hyperemic response of $261 \mathrm{~s}$. In the SHRs, after 5 min of ischemia, peak muscle arterial conductance (expressed in ml.min-1.g-1.mmHg-1) was decreased to $52.3+/-33$. vs $97.5+/-30.3$ in the WKYs $(p<0.05)$, as expected. After 30 min of ischemia, there was, in addition, a drastic shortening of the hyperemic response duration. Time to post-ischemic half normalisation of arterial conductance was $38+/-24$ s in the SHRs vs $149+/-58$ s in the WKYs $(p<0.001)$. In vivo NMR imaging of perfusion performed in calf muscle of aged WKYs and SHRs confirmed a reduced maximum peripheral vasodilation in chronically hypertensive rats. Thanks to the high temporal resolution of the technique, a new finding was demonstrated: the hyperemic response after prolonged ischemia was blunted in SHRs, the molecular mechanisms and significance of which are currently unknown.
\end{abstract}




\section{Abtsrat \#: $7-2$ \\ EFFECT OF SOME CALCIUM CHANNEL BLOCKERS IN EXPERIMENTALLY INDUCED DIABETIC NEPHROPATHY IN RATS}

Yousef, W.M.; Morsy, M.D.; Abd El-Wahed, M.M.; Ghanayem, N.M.; Omar, A.H.

Meet Khakan,32712, Shebin El Kom, Menoufiya, EGYPT

Introduction \& Aim: Diabetic nephropathy (DNP) is considered a CRD (Chronic Renal Disease); it is a major cause of illness and premature death in people with DM. Furthermore, it is considered the single most important cause of end stage renal disease in the western world and accounts for more than a quarter of all end stage renal diseases. The present study was designed to illustrate the role of CCBs (amlodipine and diltiazem) in prevention and treatment of DNP in rats. Materials \& Methods: Eighty male albino rats weighing $(130-180 \mathrm{gm})$ were used in this study. These animals were subdivided into five equal groups. Insulinopenic diabetes was induced by STZ, two weeks later, 30 minutes of complete ischaemia was induced in the left kidney to induce diabetic nephropathy then treatment was started for 12 weeks. At the end of experiment urine samples and blood samples were taken for biochemical analysis and kidneys were taken after scarification for histopathological evaluation. Results: Combination of renal ischaemia with DM produced a significant increase in rat weight, rat kidney weight, BUN (Blood Urea Nitrogen) level, K/B (Kidney/Body weight) ratio, random blood glucose, 24 hrs urine proteins, and 24 hrs urine volumes and creatinine clearance. Treatment with diltiazem or amlodipine significantly lowered elevated SBP and elevated $24 \mathrm{hrs}$ urine volumes. Furthermore, treatment with captopril produced a highly significant lowering of elevated SBP and elevated serum creatinine; and a significant reduction in elevated K/B ratio and proteinuria. Light microscopic examination of diabetic kidneys revealed glomerulopathy characterized by thickening of the glomerular basement membrane, mesangial matrix expansion, arteriolar hyalinosis and large proteinaceous deposits occluding some capillary loops and hyaline droplets within the glomeruli. Moreover, examination of kidneys of ischaemic animals by light microscope revealed focal tubular necrosis at multiple points along the nephron, interstitial edema and accumulation of leucocytes within dilated vasa recta. Conclusion: It can be concluded that, renal ischaemia hasten the progression of DNP, diltiazem and amlodipine have a tendency to reverse of changed parameters toward normal values except biochemical parameters, generally speaking, diltiazem is better than amlodipine in reversing biochemical and histopathological changes produced by DNP, and captopril reversed most of changed parameters except histopathological changes. Recommendations: Based on the obtained results from the present study, one can recommend that; 1) Diltiazem and amlodipine have a nephroprotective effect in DNP, therefore, they should be used in diabetic patients to protect and/or slow progression of DNP. 2) Captopril might be considered the first therapy for DNP. Moreover, combination of captopril with CCBs could be a more effective tool got protection and/or slow progression of DNP. 


\title{
Abtsrat \#: $7-3$ \\ IDENTICAL EFFECTS BY AP5A AND AP4A IN HUMAN RENAL AND MESENTERIC RESISTANCE ARTERIES ARE DUE TO ACTIVATION OF NON-IDENTICAL PURINORECEPTORS
}

\author{
Steinmetz* M, Janssen $\mathrm{AK}^{*}$, Pelster F, Piechota HJ, Rahn $\mathrm{KH}^{*}$, Schlatter $\mathrm{E}^{*}$ \\ * Dept. of Internal Medicine, Center of Surgery, University of Münster, Albert-Schweitzer Str. 33, 48149 \\ Münster, Germany
}

Purposes: In isolated human mesenteric (hMRA) and renal (hRRA) resistance arteries Ap5A and Ap4A are potent vasoconstrictors at basal tone and potent vasodilators after precontraction of arteries with endotheline or phenylephrine. With pharmacological receptor studies the underlying purinoreceptors should be determined, to understand the dynamic vasoactivity of these purinergic agents. Material and methods: In tissue preparations obtained from the local center of surgery small resistance arteries were isolated (\&\#61638; $215-297 \mu \mathrm{m})$. Arteries were mounted in a myograph for measurement of isometric wall tension at control conditions and after preincubation of purinoreceptor antagonists. Results and discussion: Contractions induced by Ap5A in both hMRA and hRRA are inhibited by PPADS (pyridoxalphosphate-6-azophenyl-2',4'-disulfonic acid, P2X-receptor antagonist). Ap4A-induced contractions are inhibited by PPADS in hMRA only. In both arteries Ap5A and Ap4A-induced contractions are not inhibited by ADP3'5' (P2Y purinoceptor antagonist). Thus, contractions by Ap5a and Ap4A in hMRA and by Ap5A in hRRA seem to be P2X but not P2Y receptor-mediated, whereas Ap4A induced contractions in hRRA are caused by another purinoceptor. Relaxations induced by Ap5A in hMRA cannot be inhibited with ADP3' 5 ' or with PPADS. Ap5A-induced relaxations in hRRA are inhibited by PPADS but not ADP3'5'. Ap5A-induced relaxations in hRRA are less inhibited by PPADS than Ap4A-induced relaxations. Ap4A-induced relaxations in hMRA are not inhibited by PPADS. Ap4A-induced relaxations both in hMRA and hRRA are inhibited by ADP3'5'. Thus, in hMRA and hRRA Ap4A induces relaxations via $P 2 Y$ receptor activation; the divergent antagonism of PPADS suggests different Ap4A-activated P2Y receptors in these two resistance arteries. Ap5A in hMRA and hRRA evokes relaxations via another receptor than Ap4A. Obviously, different human vascular beds dispose of heterogenous purinoreceptors being activated by different diadenosine polyphosphates without using identical receptors for evoking identical vasoactivity. 


\title{
Abtsract \#: $7-4$ \\ DIVERGING VASORELAXING EFFECTS OF C-TYPE NATRIURETIC PEPTIDE IN RENAL RESISTANCE ARTERIES AND AORTAS OF GC-A DEFICIENT MICE
}

\author{
Steinmetz $\mathrm{M}^{*}$, Potthast $\mathrm{R}$, Sabrane $\mathrm{K}$ and Kuhn $\mathrm{M}$
}

Institute of Pharmacology and Toxicology and *Department of Internal Medicine D, University Münster, Albert-Schweitzer Str. 33, 48149 Münster, Germany

Purposes: Characterization of the vasorelaxing effects of natriuretic peptides (ANP, BNP and CNP) in isolated renal resistance arteries (RRA) from wild-type mice and mice with either systemic (GC-A -/-) or smooth muscle-restricted deletion of GC-A (SMC GC-A KO) Methods, results and discussion: Isolated RRA ring segments were mounted in a myograph for measurement of isometric wall tension. In RRA from wild-type $(\mathrm{GC}-\mathrm{A}+/+)$ mice natriuretic peptides induced concentration-dependent vasorelaxations with the rank order of potency ANP>BNP>CNP. In RRA obtained from mice with systemic or smooth muscle-restricted deletion of GC-A the effects of ANP and BNP were abolished. In contrast, CNP induced concentration-dependent vasorelaxations of GC-A -/- and SMC GC-A KO RRA, demonstrating that this effects are mediated by another receptor, probably GC-B. However, the efficacy of CNP for vasorelaxation was markedly diminished $(46 \%+/-4)$ compared with wild-type RRA. Such changes in CNP reponsiveness do not affect large arteries as the aorta and they are not due to crossactivation of GC-A or vascular changes secondary to chronic arterial hypertension in GC-A -/- mice. Unaltered vasorelaxing effects of acetylcholine and sodium nitroprusside demonstrate unaltered function of downstream targests regulated by cGMP in vascular smooth muscle. An increased expression of the clearance receptor (NPR-C) or diminished expression of GC-B (quantification by real-time RT-PCR) were not found to account for the differences in CNP responsiveness. Conclusions: Observations in isolated aortic rings do not necessarily allow conclusions concerning the physiology of natriuretic peptides in the smaller resistance size arteries. Changes at the receptor level are likely to explain the diminished responsiveness to CNP in GC-A -/- and SMC GC-A KO RRA. 


\title{
Abtsract \#: $7-5$ \\ ABUNDANCE OF DEADHESION PROTEINS DURING LARGE AND SMALL ARTERY REMODELING IN L-NAME-INDUCED HYPERTENSION
}

\author{
Gilbert L-A; Girardot D; deBlois D* \& Moreau P.
}

Faculty of Pharmacy, University of Montreal, ${ }^{\star} \mathrm{CHUM}$ Research Center, Montreal, Québec, Canada.

Introduction: L-NAME-induced hypertension leads to hypertrophic remodeling in large arteries (aorta) and eutrophic remodeling in resistance arteries (mesenteric arteries), which is defined by a reduction of internal diameter without increase of the cross sectional area (CSA). Background/purposes: Matricellular proteins regulate smooth muscle cells adhesion, migration and may facilitate matrix contraction. Therefore, antiadhesive matricellular proteins, such as thrombospondine (TSP) and tenascin-C (TN-C), could be important in vascular remodeling by reducing cell-matrix interactions. Our objective was to measure the expression of TSP and TN-C in the L-NAME-induced hypertension model to determine their implication in eutrophic and hypertrophic remodeling. Material/methods: male Wistar rats were treated with L-NAME in their drinking water at a dose of $50 \mathrm{mg} / \mathrm{kg} /$ day during 0 (control), 1, 3, 7 and 14 days ( $n=5$ /group). Temporal expression of TSP and TN-C in the aorta and mesenteric arteries was determined by western immonublotting. Results: In the aorta, TSP content tended to increase at day $1(139+/-31 \%$ vs control values of $100 \%$ ) and then returned to control values within 3 days. Aortic TN-C content was generally increased during L-NAME treatment with a peak at 7 days $(488+/-196 \%)$. In mesenteric arteries, TSP expression was increased only after 14 days of L-NAME treatment $(176+/-12 \%, p<0.05)$, while TN-C content decreased progressively to reach $34+/-5 \%$ of control values $(p<0.05)$. Discussion: In L-NAME induced hypertension, vascular TSP content was increased in both hypertrophic and eutrophic remodeling, but with a different kinetic. In contrast, TN-C content was modified in opposite direction in large and small arteries, suggesting a different involvement in the two types of remodeling. These data suggest that hypertrophic remodeling may require a decrease in cell adhesion, whereas eutrophic remodeling is characterized by an initial increase of adhesion followed by re-equilibration with TSP overexpression. 


\title{
Abtsract \#: 7-6 \\ HIGH BLOOD PRESSURE REDUCTION REVERSES AT2 RECEPTOR-MEDIATED VASOCONSTRICTION INTO VASODILATION IN SPONTANEOUSLY HYPERTENSIVE RATS
}

\author{
Dong You, Laurent Loufrani\#, Khalid Matrougui, Bernard I Levy\& Robert E Widdop§, and Daniel \\ Henrion\#
}

INSERM Unit 541, and \&Dept of Physiology, AP-HP-Hôpital Lariboisière, 41 bvd de la Chapelle, Paris, 75010 France,

We have previously shown that, in normotensive rats, Angiotensin II (Ang II) type 2 receptor (AT2R) stimulation causes endothelium-dependent vasodilation which does not desensitize after chronic Angiotensin II type 1 receptor (AT1R) blockade, suggesting a role for AT2R in antihypertensive treatment. In the present study, we recorded mean arterial pressure (MAP) and investigated in vitro AT2R by western blot analysis, immunohistochemistry and function in mesenteric resistance arteries (205 $\pm 11 \mathrm{~mm}$ diameter) isolated from Wistar-Kyoto (WKY) and spontaneously hypertensive rats (SHR) receiving for 4 weeks in drinking water : placebo (control), AT1R blockade (candesartan, $2 \mathrm{mg} / \mathrm{kg} / \mathrm{d}$ ), angiotensin converting enzyme inhibitor (perindopril, $3 \mathrm{mg} / \mathrm{kg} / \mathrm{d}$ ), non-selective antihypertensive drug (hydralazine, 16 or 24 $\mathrm{mg} / \mathrm{kg} / \mathrm{d})$ or candesartan + hydralazine $(16 \mathrm{mg} / \mathrm{kg} / \mathrm{d})$. In precontracted isolated arteries $(54 \pm 4 \mathrm{~mm}$, diameter decrease) AT2R stimulation (Ang II in the presence of candesartan) caused vasodilation ( $24 \pm 3 \mathrm{~mm}$ diameter increase) in WKY rats (MAP $=118 \pm 8 \mathrm{mmHg}$ ) and vasoconstriction (8 $\pm 3 \mathrm{~mm}$ diameter decrease) in SHR $(M A P=183 \pm 11 \mathrm{mmHg})$. In SHR treated with candesartan $(M A P=146 \pm 8 \mathrm{mmHg})$ or hydralazine $(16$ $\mathrm{mg} / \mathrm{kg} / \mathrm{d}$; MAP $=145 \pm 6 \mathrm{mmHg}$ ) AT2R-induced contraction was reduced by $50 \%$. In SHR treated with perindopril (MAP $=125 \pm 11 \mathrm{mmHg}$ ), AT2R stimulation induced a small but significant vasodilation. In SHR treated with hydralazine $(24 \mathrm{mg} / \mathrm{kg} / \mathrm{d}$; MAP $=105 \pm 10 \mathrm{mmHg})$ and in SHR treated with hydralazine $(16 \mathrm{mg} / \mathrm{kg} / \mathrm{d})+$ candesartan $(\mathrm{MAP}=102 \pm 9 \mathrm{mmHg})$ an $A T 2 \mathrm{R}$ mediated-vasodilation was completely restored. Immunochemistry and Western-blot analysis showed that AT2R expression was lower in SHR than in WKY. Treatments reducing arterial pressure in SHR to normal level restored both AT2R mediated-vasodilation and AT2R expression in mesenteric arteries. Our results suggest that in resistance arteries of SHR 1) AT2R is down regulated by hypertension and 2) specific and non-specific anti-hypertensive treatments restore AT2R expression and vasodilator functions. Keywords: angiotensin; receptors, vascular; vasodilation; hypertension. 


\section{Abtsract \#: $7-7$ \\ INTERMITTENT HYPOXIA INDUCES NON CONCOMITTANT CHANGES IN BLOOD PRESSURE AND VASCULAR REACTIVITY IN MICE}

Julien C; Dematteis M; Lévy P

Laboratoire "Hypoxie-Physiopathologie", Faculté de Médecine, 38706 La Tronche, France

Intermittent hypoxia $(\mathrm{IH})$ is one hallmark of obstructive sleep apnea syndrome that may be responsible for systemic hypertension and increased vascular reactivity. We previously demonstrated in mice exposed to $\mathrm{IH}$ an augmented vascular response to norepinephrine (NE), in particular when sympathetic nervous system (SNS) was activated. Conversely response to acetylcholine (Ach) was unchanged. The pathophysiology underlying such changes is still unknown (increased sympathetic activity, decreased NE reuptake and/or increased adrenergic receptors number of affinity). Moreover the chronological relationship between changes in blood pressure and vascular reactivity remains to be determined. Blood pressure (BP), heart rate (HR) and vascular reactivity (VR) were assessed in C57BL/6 male mice $(20 \mathrm{~g})$ exposed to IH (normoxia-hypoxia: 21-4\% FiO2, 2 cycles/min, 8 h/day, 14 or 35 days) or normoxia (sham animals). BP and HR were measured for 7 hours in animals equipped with an arterial catheter and exposed to IH or air, for 1 or 14 days ( $n=16$ per group). VR was assessed in perfused hindquarters (mice exposed to $\mathrm{IH}$ or air for 14 or 35 days, $\mathrm{n}=8$ per group). Vasoconstrictor responses to NE were assessed in different experimental conditions: with active SNS and NE reuptake under isoflurane anesthesia, then after inhibition of NE reuptake by desipramine perfusion, and finally after SNS inactivation by euthanasia. Similarly, vasodilator responses to Ach were assessed in euthanized mice to study the endothelium dependent vasodilation before and after L-NAME treatment. Except the transient fluctuations observed during each cycle of $\mathrm{IH}$, no permanent changes in $\mathrm{BP}$ and $\mathrm{HR}$ were observed the first day of $\mathrm{IH}$. After 14 days, BP significantly increased compared to sham mice $(+21 \mathrm{mmHg})$. The highest increase in BP (both systolic and diastolic BP) was observed the first 2 hours of IH exposure. Then diastolic BP progressively decreased to reach sham level, while systolic BP remained elevated and HR was unchanged. Vascular response to NE increased only after 35 days of $\mathrm{IH}$, and only when SNS was active. Conversely, no significant changes in Ach vasodilation were observed. IH induced systemic hypertension with unchanged $H R$, and delayed modification of vascular reactivity involving SNS activity. The hypertension was systolo-diastolic, except during $\mathrm{IH}$ exposure: systolic hypertension persisted while diastolic BP progressively normalized. This differential regulation of BP suggests i) neurogenic regulation (sympathetic hyperactivity) plus increased blood volume, the latter could underlie the persisting systolic hypertension, ii) humoral regulation related to hypoxia-reoxygenation leading to decreased vascular resistance. 


\title{
Abtsrat \#: $7-8$ \\ EFFECTS OF REDUCED UTERO-PLACENTAL PERFUSION ON GLOMERULAR BIOLOGY IN THE ADULT RAT OFFSPRING
}

\author{
Sanders MW; Fazzi GE, Jansse GMJ, Blanco CE*, De Mey JGR.
}

Dept. of Pharmacology \& Toxicology, Cardiovascular Research Institute Maastricht (CARIM), *Dept. of Pediatrics, Research Institute Growth and Development (GROW), University of Maastricht, PO box 616, 6200 MD, Maastricht, The Netherlands.

Introduction: Intrauterine malnutrition and hypoxia increase the risk for the development of cardiovascular disease in the adult. We studied the effects of intrauterine stress (IUS) on renal glomerular structure and function and on blood pressure in adult Wistar rats. Material \& methods: IUS was induced by bilateral ligation of the uterine arteries at day 17 of pregnancy. In part of the IUS and control animals a unilateral nephrectomy (left) was performed at 21 days of age. At the age of 12 weeks, half of the rats were given high salt drinking water $(2 \% \mathrm{NaCl})$ for 4 weeks. At the age of 16 weeks, the femoral artery and vein were cannulated for blood pressure monitoring, blood samplingand, infusion of artificial rat plasma, $\mathrm{PAH}$ (para-aminohippurate; renal blood flow) and inulin (glomerular filtration rate). Results: After IUS, litter size $(5 \pm 1$ vs. $12 \pm 1)$ and birth weight were reduced $(5.08 \pm 0.06 \mathrm{~g}$ vs. $5.33 \pm 0.05 \mathrm{~g})$, while hematocrit at birth was increased $(43.08 \pm 0.64 \%$ vs. $38.36 \pm 0.57 \%)$. At 16 weeks after IUS, body weight and hematocrit were not different from control animals, while renal morphometry showed an increased glomerular size and a reduced number of glomeruli (\&\#8776; -30\%). The filtration fraction (filtration/perfusion ratio) was significantly increased 16 weeks after IUS, because renal perfusion was increased by $36 \%$ and glomerular filtration rate was increased by $114 \%$. Kidney function of 16 -week-old control rats was not affected by removal of the left kidney at 21 days of age. The right kidney compensated for the loss of the other (left) kidney. After intrauterine stress, the remaining right kidney failed to compensate for the loss of the left kidney and blood pressure was increased. High salt did not change renal function and blood pressure in control animals. However, after IUS, renal blood flow, glomerular filtration rate and the filtration fraction were decreased and blood pressure was increased. Discussion: A suboptimal fetal micro-environment impairs renal development, which results in a persistent reduction of renal reserve. 


\title{
Ahtsract \#: $7-9$ \\ ROLE OF ETB RECEPTORS IN THE RAT PULMONARY VASOCONSTRICTION INDUCED BY ET-1
}

\author{
Sauvageau S; Thorin E; Caron A; Dupuis J
}

Université de Montréal, Institut de Cardiologie, Centre de recherche, 5000 rue Bélanger, Montréal, Québec, H1T 1C8, Canada

Introduction. Endothelin-1 (ET) is a potent pulmonary vasoconstrictor. The ETA and ETB receptors are present on smooth muscle and induce vasoconstriction. The ETB is also present on the endothelium where it induces the release of the vasodilators nitric oxide and prostacyclin, as well as the potent pulmonary vasoconstrictor tromboxane A2. The vascular distribution and biology of ETB receptors may have important implications. Purpose. To evaluate the respective roles of the ETA and ETB receptors as well as of the endothelium on ET-induced pulmonary vasoconstriction in rats. Methods. Lungs were isolated at a constant flow rate of $10 \mathrm{ml} / \mathrm{min}$ and changes in pressure to concentrations (10 $\mathrm{nM}$ to $300 \mathrm{nM}$ ) of ET and sarafotoxin $6 \mathrm{c}$ (S6c) were measured, the former with and without Bosentan (0.1 mM) or meclofenamic acid $(0.1 \mathrm{mM})$. A second set of experiments was conducted on myographs with isolated pulmonary arteries (150 to 200 micron diameter). Maximal vasoconstriction was determined after $127 \mathrm{mM}$ $\mathrm{KCl}$. Preparations were also subjected to cumulative concentration-response curves to ET and S6c, with or without endothelium. Results. In isolated lungs, both ET (36.5+/-6.4 mmHg delta pressure, mean+/-SEM) and S6c (35.8+/-7.6 mmHg) induced important and similar vasoconstrictions with the formation of severe pulmonary oedema. Bosentan suppressed the response to $E T(p=0.001)$ and the development of oedema. Meclofenamic acid partially antagonized ET-mediated vasoconstriction $(p=0.001)$. In isolated arteries, the maximal vasoconstriction induced by ET (Emax $91+/-4 \%$, EC50 $11.0+/-2.2 \mathrm{nM})$ and S6c (Emax 85+/-4\%, EC50 5.0+/-1.6 nM) were also not significantly different. Removal of the endothelium did not significantly modify the maximal response to ET $(78.3+/-6.3 \%)$ and to S6c (91.1+/-3.7\%) and also did not affect EC50 values. Conclusion. In the normal rat, ET-induced pulmonary vasoconstriction of small arteries is essentially mediated by the ETB receptor with no significant modulator role of the endothelium. In isolated lungs, ET also releases vasoconstrictive cyclooxygenase products. Further work is necessary to elucidate the mechanisms of ET-induced contraction in the lungs of various species. 